\title{
Search for chargino and neutralino production in final states with a Higgs boson and missing transverse momentum at $\sqrt{s}=13 \mathrm{TeV}$ with the ATLAS detector
}

\author{
M. Aaboud et al. \\ (ATLAS Collaboration)
}

(Received 27 December 2018; published 30 July 2019)

\begin{abstract}
A search is conducted for the electroweak pair production of a chargino and a neutralino $p p \rightarrow \tilde{\chi}_{1}^{ \pm} \tilde{\chi}_{2}^{0}$, where the chargino decays into the lightest neutralino and a $W$ boson, $\tilde{\chi}_{1}^{ \pm} \rightarrow \tilde{\chi}_{1}^{0} W^{ \pm}$, while the neutralino decays into the lightest neutralino and a Standard Model-like $125 \mathrm{GeV}$ Higgs boson, $\tilde{\chi}_{2}^{0} \rightarrow \tilde{\chi}_{1}^{0} h$. Fully hadronic, semileptonic, diphoton, and multilepton (electrons, muons) final states with missing transverse momentum are considered in this search. Higgs bosons in the final state are identified by either two jets originating from bottom quarks $(h \rightarrow b \bar{b})$, two photons $(h \rightarrow \gamma \gamma)$, or leptons from the decay modes $h \rightarrow W W, h \rightarrow Z Z$ or $h \rightarrow \tau \tau$. The analysis is based on $36.1 \mathrm{fb}^{-1}$ of $\sqrt{s}=13 \mathrm{TeV}$ proton-proton collision data recorded by the ATLAS detector at the Large Hadron Collider. Observations are consistent with the Standard Model expectations, and 95\% confidence-level limits of up to $680 \mathrm{GeV}$ in $\tilde{\chi}_{1}^{ \pm} / \tilde{\chi}_{2}^{0}$ mass are set in the context of a simplified supersymmetric model.
\end{abstract}

DOI: 10.1103/PhysRevD.100.012006

\section{INTRODUCTION}

Theoretical and experimental arguments suggest that the Standard Model (SM) is an effective theory valid up to a certain energy scale. The observation by the ATLAS and CMS collaborations of a particle consistent with the SM Higgs boson [1-4] has brought renewed attention to the mechanism of electroweak symmetry breaking and the hierarchy problem [5-8]: the Higgs boson mass is strongly sensitive to quantum corrections from physics at very high energy scales and demands a high level of fine-tuning. Supersymmetry (SUSY) [9-14] resolves the hierarchy problem by introducing for each known boson or fermion a new partner (superpartner) that shares the same mass and internal quantum numbers if supersymmetry is unbroken. However, these superpartners have not been observed, so SUSY must be a broken symmetry and the mass scale of the supersymmetric particles is as yet undetermined. The possibility of a supersymmetric dark matter $(\mathrm{DM})$ candidate $[15,16]$ is related closely to the conservation of $R$-parity [17]. Under the $R$-parity

*Full author list given at the end of the article.

Published by the American Physical Society under the terms of the Creative Commons Attribution 4.0 International license. Further distribution of this work must maintain attribution to the author(s) and the published article's title, journal citation, and DOI. Funded by SCOAP ${ }^{3}$. conservation hypothesis, the lightest supersymmetric particle (LSP) is stable. If the LSP is weakly interacting, it may provide a viable DM candidate. The nature of the LSP is defined by the mechanism that spontaneously breaks supersymmetry and the parameters of the chosen theoretical framework.

In the SUSY scenarios considered as benchmarks in this paper, the LSP is the lightest of the neutralinos $\left(\tilde{\chi}^{0}\right)$ which, together with the charginos $\left(\tilde{\chi}^{ \pm}\right)$, represent the mass eigenstates formed from the mixture of the $\gamma, W, Z$ and Higgs bosons' superpartners (the higgsinos, winos and binos). The neutralinos and charginos are collectively referred to as electroweakinos. Specifically, the electroweakino mass eigenstates are designated in order of increasing mass as $\tilde{\chi}_{i}^{ \pm}(i=1,2)$ (charginos) and $\tilde{\chi}_{j}^{0}$ $(j=1,2,3,4)$ (neutralinos). In the models considered in this paper, the compositions of the lightest chargino $\left(\tilde{\chi}_{1}^{ \pm}\right)$ and next-to-lightest neutralino $\left(\tilde{\chi}_{2}^{0}\right)$ are wino-like and the two particles are nearly mass degenerate, while the lightest neutralino $\left(\tilde{\chi}_{1}^{0}\right)$ is assumed to be bino-like.

Naturalness considerations [18,19] suggest that the lightest of the charginos and neutralinos have masses near the electroweak scale. Their direct production may be the dominant mechanism at the Large Hadron Collider (LHC) if the superpartners of the gluon and quarks are heavier than a few TeV. In SUSY models where the masses of the heaviest (pseudoscalar, charged) MSSM Higgs boson and the superpartners of the leptons have masses larger than 


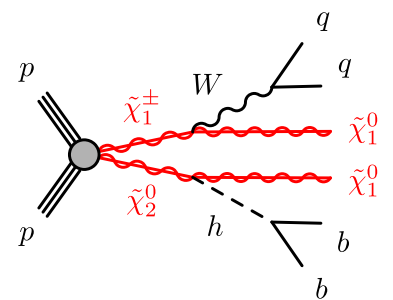

(a) $0 \ell b \bar{b}$

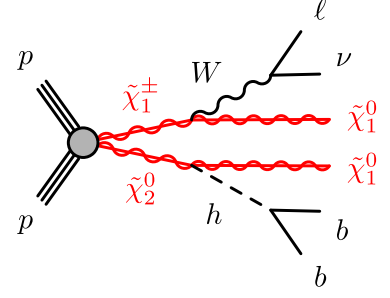

(b) $1 \ell b \bar{b}$

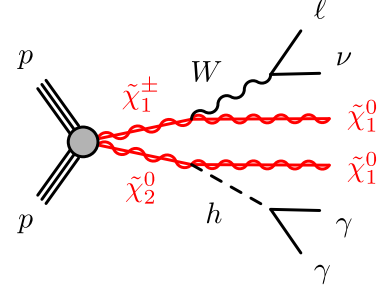

(c) $1 \ell \gamma \gamma$

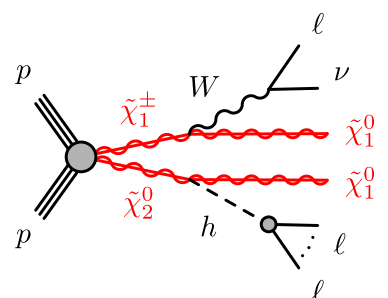

(d) $\ell^{ \pm} \ell^{ \pm}, 3 \ell$

FIG. 1. Diagrams illustrating the signal scenarios considered for the pair production of chargino and next-to-lightest neutralino targeted by the (a) hadronic $(0 \ell b \bar{b})$ and (b) $1 \ell b \bar{b}$, (c) $1 \ell \gamma \gamma$, (d) $\ell^{ \pm} \ell^{ \pm}, 3 \ell$ leptonic channel selections. In (a) and (b) the Higgs boson decays into two $b$-quarks. In (c), the diphoton channel is shown with $h \rightarrow \gamma \gamma$. In (d), the visible multilepton final state of the Higgs boson is shown. Leptons are either electrons or muons.

those of the lightest chargino and next-to-lightest neutralino, the former might decay into the $\tilde{\chi}_{1}^{0}$ and a $W$ boson $\left(\tilde{\chi}_{1}^{ \pm} \rightarrow W \tilde{\chi}_{1}^{0}\right)$, while the latter could decay into the $\tilde{\chi}_{1}^{0}$ and the lightest MSSM Higgs boson ( $h$, SM-like), or $Z$ boson $\left(\tilde{\chi}_{2}^{0} \rightarrow h / Z \tilde{\chi}_{1}^{0}\right)[17,20,21]$. The decay via the Higgs boson is dominant for many choices of the parameters as long as the mass-splitting between the two lightest neutralinos is larger than the Higgs boson mass and the higgsinos are heavier than the winos. SUSY models of this kind, where sleptons are not too heavy although with masses above that of $\tilde{\chi}_{1}^{ \pm}$ and $\tilde{\chi}_{2}^{0}$, could provide a possible explanation for the discrepancy between measurements of the muon's anomalous magnetic moment $g-2$ and SM predictions [22-25].

This paper presents a search in proton-proton collision produced at the LHC at a center-of-mass energy $\sqrt{s}=$ $13 \mathrm{TeV}$ for the direct pair production of mass-degenerate charginos and next-to-lightest neutralinos that promptly decay as $\tilde{\chi}_{1}^{ \pm} \rightarrow W \tilde{\chi}_{1}^{0}$ and $\tilde{\chi}_{2}^{0} \rightarrow h \tilde{\chi}_{1}^{0}$. The search targets hadronic and leptonic decays of both the $W$ and Higgs bosons. Three Higgs decay modes are considered: decays into a pair of $b$-quarks, a pair of photons, or a pair of $W$ or $Z$ bosons or $\tau$-leptons, where at least one of the $W / Z / \tau$ decays leptonically. Four signatures are considered, illustrated in Fig. 1. All final states contain missing transverse momentum ( $\vec{p}_{\mathrm{T}}^{\text {miss }}$, with magnitude $E_{\mathrm{T}}^{\text {miss }}$ ) from neutralinos, and in some cases neutrinos. Events are characterized by the various decay modes of the $W$ and Higgs bosons. The signatures considered have the following: jets, with two of them originating from the fragmentation of $b$-quarks, called $b$-jets, and either no leptons $[0 \ell b \bar{b}$, Fig. 1(a)], or exactly one lepton $(\ell=e, \mu)[1 \ell b \bar{b}$, Fig. 1(b)]; two photons and one lepton [1 $\ell \gamma \gamma$, Fig. 1(c)]; only leptons [Fig. 1(d)] such that the final state contains either two leptons with the same electric charge, $\ell^{ \pm} \ell^{ \pm}$, or three leptons, $3 \ell$.

A simplified SUSY model [26,27] is considered for the optimization of the search and the interpretation of results. The $\tilde{\chi}_{1}^{ \pm} \rightarrow W \tilde{\chi}_{1}^{0}$ and $\tilde{\chi}_{2}^{0} \rightarrow h \tilde{\chi}_{1}^{0}$ decays are assumed to have $100 \%$ branching ratio. The Higgs boson mass is set to $125 \mathrm{GeV}$ and its branching ratios are assumed to be the same as in the SM. The Higgs boson candidate can be fully reconstructed with $0 \ell b \bar{b}, 1 \ell b \bar{b}$ and $1 \ell \gamma \gamma$ signatures, while $\ell^{ \pm} \ell^{ \pm}$and $3 \ell$ final states are sensitive to decays $h \rightarrow W W, h \rightarrow Z Z$ and $h \rightarrow \tau \tau$. Previous searches for charginos and neutralinos at the LHC targeting decays via the Higgs boson into leptonic final states have been reported by the ATLAS [28] and CMS [29] collaborations; a search in the hadronic channel is also reported in this paper.

\section{ATLAS DETECTOR}

The ATLAS detector [30] is a multipurpose particle detector with a forward-backward symmetric cylindrical geometry and nearly $4 \pi$ coverage in solid angle. ${ }^{1}$ The inner tracking detector consists of pixel and microstrip silicon detectors covering the pseudorapidity region $|\eta|<2.5$, surrounded by a transition radiation tracker which enhances electron identification in the region $|\eta|<2.0$. A new inner pixel layer, the insertable B-layer [31,32], was added at a mean radius of $3.3 \mathrm{~cm}$ during the period between Run 1 and Run 2 of the LHC. The inner detector is surrounded by a thin superconducting solenoid providing an axial $2 \mathrm{~T}$ magnetic field and by a fine-granularity lead/liquid-argon (LAr) electromagnetic calorimeter covering $|\eta|<3.2$. A steel/scintillator-tile calorimeter provides hadronic coverage in the central pseudorapidity range $(|\eta|<1.7)$. The end cap and forward regions $(1.5<|\eta|<4.9)$ of the

\footnotetext{
${ }^{1}$ ATLAS uses a right-handed coordinate system with its origin at the nominal interaction point in the center of the detector. The positive $x$ axis is defined by the direction from the interaction point to the center of the LHC ring, with the positive $y$ axis pointing upwards, while the beam direction defines the $z$ axis. Cylindrical coordinates $(r, \phi)$ are used in the transverse plane, $\phi$ being the azimuthal angle around the $z$ axis. The pseudorapidity $\eta$ is defined in terms of the polar angle $\theta$ by $\eta=-\ln \tan (\theta / 2)$. Rapidity is defined as $y=0.5 \ln \left[\left(E+p_{z}\right) /\left(E-p_{z}\right)\right]$ where $E$ denotes the energy and $p_{z}$ is the component of the momentum along the beam direction. The angular distance $\Delta R$ is defined as $\sqrt{(\Delta y)^{2}+(\Delta \phi)^{2}}$.
} 
TABLE I. List of generators used for the different processes. Information is given about the underlying-event tunes, the PDF sets and the perturbative QCD highest-order accuracy (LO, NLO, next-to-next-to-leading order, NNLO, and next-to-next-to-leading-log, NNLL) used for the normalization of the different samples.

\begin{tabular}{|c|c|c|c|c|}
\hline Process & Generator + fragmentation/hadronization & Tune & PDF set & Cross-section \\
\hline$W / Z+$ jets & SHERPA-2.2.1 [47] & Default & NNPDF3.0NNLO [42] & NNLO \\
\hline$t \bar{t}$ & $\begin{array}{l}\text { Powheg-Box v2 }[49,50] \\
+ \text { PyTHIA-6.428 }[53]\end{array}$ & PERUGIA2012 [51] & CT10 [52] & $\begin{array}{l}\text { NNLO } \\
+ \text { NNLL }\end{array}$ \\
\hline Single top & $\begin{array}{l}\text { PowHEG-Box v1 or v2 } \\
+ \text { PYTHIA- } 6.428\end{array}$ & PERUGIA2012 & CT10 & $\begin{array}{l}\text { NNLO } \\
+ \text { NNLL }\end{array}$ \\
\hline $\begin{array}{l}\text { Diboson } \\
W W, W Z, Z Z \\
t \bar{t}+X\end{array}$ & SHERPA-2.2.1 & Default & NNPDF3.0NNLO & NLO \\
\hline $\begin{array}{l}t \bar{t} W / Z \\
4 \text { top quarks }\end{array}$ & $\begin{array}{l}\text { MADGRAPH-2.2.2 [38] } \\
+ \text { PYTHIA-8.186 [39] }\end{array}$ & A14 [40] & NNPDF2.3 & NLO \\
\hline $\bar{t} \bar{t} h$ & $\begin{array}{l}\text { MADGRAPH5_aMC@NLO-2.2.1 } \\
+ \text { HERWIG++-2.7.1 }\end{array}$ & UEEE5 [54] & CT10 & NLO \\
\hline$W h, Z h$ & PYTHIA-8.186 [48] & A14 & NNPDF2.3 & NLO \\
\hline
\end{tabular}

hadronic calorimeter are made of LAr active layers with either copper or tungsten as the absorber material. A muon spectrometer with an air-core toroid magnet system surrounds the calorimeters. Three layers of high-precision tracking chambers provide coverage in the range $|\eta|<2.7$, while dedicated fast chambers allow triggering in the region $|\eta|<2.4$. The ATLAS trigger system consists of a hardware-based level-1 trigger followed by a softwarebased high-level trigger [33].

\section{DATA AND MONTE CARLO SIMULATION}

The data used in this analysis were collected in $p p$ collisions at the LHC with a center-of-mass energy of $13 \mathrm{TeV}$ and a $25 \mathrm{~ns}$ proton bunch crossing interval during 2015 and 2016. The full dataset corresponds to an integrated luminosity of $36.1 \mathrm{fb}^{-1}$ after requiring that all detector subsystems were operational during data recording. The uncertainty in the combined $2015+2016$ integrated luminosity is $2.1 \%$. It is derived, following a methodology similar to that detailed in Ref. [34], and using the LUCID-2 detector for the baseline luminosity measurements [35], from calibration of the luminosity scale using $x-y$ beam-separation scans. Each event includes on average 13.7 and 24.9 inelastic $p p$ collisions in the same bunch crossing (pileup) in the 2015 and 2016 datasets, respectively. In the $0 \ell b \bar{b}$ and $1 \ell b \bar{b}$ channels, events are required to pass $E_{\mathrm{T}}^{\text {miss }}$ triggers with period-dependent thresholds. These triggers are fully efficient for events with $E_{\mathrm{T}}^{\text {miss }}>200 \mathrm{GeV}$ reconstructed offline. Data for the $1 \ell \gamma \gamma$ signature were collected with a diphoton trigger which selects events with at least two photons, with transverse momentum thresholds on the highest- and second-highest $p_{\mathrm{T}}$ photons of $35 \mathrm{GeV}$ and $25 \mathrm{GeV}$, respectively. A combined set of dilepton and single-lepton triggers was used for event selection in the $\ell^{ \pm} \ell^{ \pm}$and $3 \ell$ channels.
Monte Carlo (MC) samples of simulated events are used to model the signal and to aid in the estimation of SM background processes, with the exception of multijet processes, which are estimated from data. All simulated samples were produced using the ATLAS simulation infrastructure [36] and GEANT4 [37], or a faster simulation based on a parameterization of the calorimeter response and GEANT4 for the other detector systems. The simulated events were reconstructed with the same algorithm as that used for data.

SUSY signal samples were generated with MAdGraph5_aMC@NLO v2.2.3 [38] (v2.3.3 for $0 \ell b \bar{b}$ ) at leading order (LO) and interfaced to PYTHIA v8.186 [39] (v8.212 for $0 \ell b \bar{b}$ ) with the A14 [40] set of tuned parameters (tune) for the modeling of the parton showering (PS), hadronization and underlying event. The matrix element (ME) calculation was performed at tree level and includes the emission of up to two additional partons. The ME-PS matching was done using the CKKW-L [41] prescription, with a matching scale set to one quarter of the chargino and next-to-lightest neutralino mass. The NNPDF23LO [42] parton distribution function (PDF) set was used. The cross sections used to evaluate the signal yields are calculated to next-to-leading-order (NLO) accuracy in the strong coupling constant, adding the resummation of soft gluon emission at next-to-leading-logarithm accuracy (NLO + NLL) [43-45]. The nominal cross section and its uncertainty are taken as the midpoint and half-width of an envelope of cross-section predictions using different PDF sets and factorization and renormalization scales, as described in Ref. [46].

Background samples were simulated using different MC event generators depending on the process. All background processes are normalized to the best available theoretical calculation of their respective cross sections. The event generators, the accuracy of theoretical cross sections, the 
underlying-event parameter tunes, and the PDF sets used in simulating the SM background processes are summarized in Table I. For all samples, except those generated using SHERPA [47], the EvTGEN v1.2.0 [48] program was used to simulate the properties of the bottom- and charm-hadron decays. Several samples produced without detector simulation are employed to estimate systematic uncertainties associated with the specific configuration of the MC generators used for the nominal SM background samples. They include variations of the renormalization and factorization scales, the CKKW-L matching scale, as well as different PDF sets and fragmentation/hadronization models. Details of the MC modeling uncertainties are discussed in Sec. VII.

\section{EVENT RECONSTRUCTION AND OBJECT DEFINITIONS}

Common event-quality criteria and object reconstruction definitions are applied for all analysis channels, including standard data-quality requirements to select events taken during optimal detector operation. In addition, each analysis channel applies selection criteria that are specific to the objects and kinematics of interest in those final states, which are described in Sec. VI.

Events are required to have at least one primary vertex, defined as the vertex associated with at least two tracks with $p_{\mathrm{T}}>0.4 \mathrm{GeV}$ and with the highest sum of squared transverse momenta of associated tracks [55]. Quality criteria are imposed to reject events that contain at least one jet arising from noncollision sources or detector noise [56].

Electron candidates are reconstructed from energy clusters in the electromagnetic calorimeter and inner-detector tracks. They are required to satisfy the loose likelihood identification criteria, have B-layer hits (the loose requirement), and be isolated [57,58]. These identification criteria are based on several properties of the electron candidates, including calorimeter-based shower shapes, inner-detector track hits and impact parameters, and comparisons of calorimeter cluster energy to track momentum. Corrections for energy contributions due to pileup are included. For all but the $1 \ell \gamma \gamma$ channel, electrons are also required to have $p_{\mathrm{T}}>20 \mathrm{GeV}$ and $|\eta|<2.47$; for the $1 \ell \gamma \gamma$ channel they are required to have $p_{\mathrm{T}}>15 \mathrm{GeV}$ and $|\eta|<2.37$. These electrons are used in the overlap removal procedure that is described below, and to apply lepton selections and vetoes in the various analysis channels, in some cases with additional selections applied.

Photon candidates are reconstructed from energy clusters in the electromagnetic calorimeter [59] in the region $|\eta|<2.37$, after removing the transition region between barrel and end cap calorimeters, $1.37<|\eta|<1.52$. Photons are classified as unconverted photons if they do not have tracks from a conversion vertex matched to the cluster, and as converted if they do [60]. Identification criteria are applied to separate photon candidates from $\pi^{0}$ or other neutral hadrons decaying into two photons [59]. Strict identification requirements based on calorimeter shower shapes are used to identify the so-called tight photons, which are used in the $1 \ell \gamma \gamma$ analysis channel. In this case, photons are required to satisfy an isolation criterion based on the sum of the calorimeter energy in a cone of $\Delta R=0.4$ centered on the direction of the candidate photon, minus the energy of the photon candidate itself and energy expected from pileup interactions. The resulting isolation transverse energy is required to be less than $2.45 \mathrm{GeV}+0.022 \times E_{T}^{\gamma}$, where $E_{\mathrm{T}}^{\gamma}$ is the candidate photon's transverse energy. Photons are calibrated using comparisons of data with MC simulation [57] and required to have $E_{\mathrm{T}}>25 \mathrm{GeV}$. For both the electrons and photons, additional criteria are applied to remove poor quality or fake electromagnetic clusters resulting from instrumental problems.

Muon candidates are reconstructed from matching tracks in the inner detector and muon spectrometer. They are required to meet medium quality and identification criteria and to be isolated, as described in Ref. [61], and to have $p_{\mathrm{T}}>20 \mathrm{GeV}\left(p_{\mathrm{T}}>10 \mathrm{GeV}\right.$ for the $1 \ell \gamma \gamma$ analysis $)$ and $|\eta|<2.5$. These muons are used in the overlap removal procedure and to apply lepton selections and vetoes in the various analysis channels, in some cases with additional selections applied. Events containing muons from calorimeter punch-through or poorly measured tracks are rejected if any muon has a large relative $q / p$ error, or $\sigma(q / p) /|q / p|>0.2$, where $q$ is the charge of the track and $p$ is the momentum. Cosmic-ray muons are rejected after the muon-jet overlap removal by requiring the transverse and longitudinal impact parameters to be $\left|d_{0}\right|<0.25 \mathrm{~mm}$ and $\left|z_{0} \sin \theta\right|<0.5 \mathrm{~mm}$, respectively.

Jets are reconstructed from three-dimensional topological energy clusters [62] in the calorimeter using the anti- $k_{t}$ jet algorithm [63] with a radius parameter of 0.4. Each topological cluster is calibrated to the electromagnetic scale prior to jet reconstruction. The reconstructed jets are then calibrated to the energy scale of stable final state particles ${ }^{2}$ in the MC simulation by a jet energy scale (JES) correction derived from $\sqrt{s}=13 \mathrm{TeV}$ data and simulations [64]. Further selections are applied to reject jets within $|\eta|<2.4$ that originate from pileup interactions by means of a multivariate algorithm using information about the tracks matched to each jet $[64,65]$. Candidate jets are required to have $p_{\mathrm{T}}>20 \mathrm{GeV}$ and $|\eta|<2.8$.

A jet is tagged as a $b$-jet by means of a multivariate algorithm called MV2c10 using information about the impact parameters of inner-detector tracks matched to the jet, the presence of displaced secondary vertices, and the reconstructed flight paths of $b$ - and $c$-hadrons inside the jet [66-68]. Jets tagged as $b$-jets must have $|\eta|<2.5$. Several

\footnotetext{
${ }^{2}$ Stable particles in the MC simulation event record are those that have a lifetime $\tau$ such that $c \tau>10 \mathrm{~mm}$. Jets of this kind are referred to as particle jets.
} 
operating points are available, corresponding to various efficiencies obtained in $t \bar{t}$ simulated events. The $77 \%$ efficiency point was found to be optimal for most SUSY models considered in this paper and is used in the analysis. This configuration corresponds to a background rejection of 6 for $c$-jets, 22 for $\tau$-leptons and 134 for light-quark and gluon jets [66-68], estimated using $t \bar{t}$ simulated events.

The $E_{\mathrm{T}}^{\text {miss }}$ in the event is defined as the magnitude of the negative vector sum of the $p_{\mathrm{T}}$ of all selected and calibrated physics objects in the event, with an extra term added to account for soft energy in the event that is not associated with any of the selected objects. This soft term is calculated from inner-detector tracks matched to the primary vertex to make it more resilient to pileup contamination [69].

Overlaps between reconstructed objects are accounted for and removed in a prioritized sequence. If a reconstructed muon shares an inner-detector track with an electron, the electron is removed. Jets within $\Delta R=0.2$ of an electron are removed. Electrons that are reconstructed within $\Delta R=0.4$ of any surviving jet are then removed, except in the case of the $0 \ell b \bar{b}$ channel, where $\Delta R=$ $\min \left(0.4,0.04+10 \mathrm{GeV} / p_{\mathrm{T}}^{e}\right)$, thereby allowing a high- $p_{\mathrm{T}}$ electron to be slightly closer to a jet than $\Delta R=0.4$. If a jet is reconstructed within $\Delta R=0.2$ of a muon and the jet has fewer than three associated tracks or the muon energy constitutes a large fraction $(>50 \%)$ of the jet energy, then the jet is removed. Muons reconstructed within a cone of size $\Delta R=\min \left(0.4,0.04+10 \mathrm{GeV} / p_{\mathrm{T}}^{\mu}\right)$ around the axis of any surviving jet are removed. If an electron (muon) and a photon are found within $\Delta R=0.4$, the object is interpreted as electron (muon) and the overlapping photon is removed from the event. Finally, if a jet and a photon are found within $\Delta R<0.2$, the object is interpreted as photon and the overlapping jet is removed from the event; otherwise, if $\Delta R<0.4$, the object is interpreted as a jet and the overlapping photon is discarded.

\section{KINEMATIC REQUIREMENTS AND EVENT VARIABLES}

Different analysis channels' signal regions are optimized to target different mass hierarchies of the particles involved. The event selection criteria are defined on the basis of kinematic requirements for the objects described in the previous section and event variables are presented below. In the following, jets are ordered according to decreasing $p_{\mathrm{T}}$, and $p_{\mathrm{T}}$ thresholds depend on the analysis channel.

(a) $N_{\text {jet }}$ is the number of jets with $|\eta|<2.8$ and $p_{\mathrm{T}}$ above an analysis-dependent $p_{\mathrm{T}}$ threshold.

(b) $N_{b \text {-jet }}$ is the number of $b$-jets with $|\eta|<2.5$ with $p_{\mathrm{T}}$ above an analysis-dependent $p_{\mathrm{T}}$ threshold.

(c) $\Delta \eta_{\ell \ell}$ is the pseudorapidity difference between the two leading leptons in the case of multilepton channels.

(d) The minimum azimuthal angle $\Delta \phi_{\text {min }}^{4 j}$ between the $\vec{p}_{\mathrm{T}}^{\text {miss }}$ and the $\vec{p}_{\mathrm{T}}$ of each of the four leading jets in the event is useful for rejecting events with mismeasured jet energies leading to $E_{\mathrm{T}}^{\text {miss }}$ in the event, and is defined as

$$
\Delta \phi_{\text {min }}^{4 j}=\min _{i \leq 4} \Delta \phi\left(\vec{p}_{\mathrm{T}}^{\text {miss }}, \vec{p}_{\mathrm{T}, i}^{\mathrm{jet}}\right)
$$

where $\min _{i \leq 4}$ selects the jet the minimizes $\Delta \phi$.

(e) The effective mass $m_{\text {eff }}$ is defined as the scalar sum of the $p_{\mathrm{T}}$ of jets, leptons and $E_{\mathrm{T}}^{\text {miss }}$, which aids in establishing the mass scale of the processes being probed, and is defined as

$$
m_{\mathrm{eff}}=\sum_{i}^{N_{\mathrm{jet}}} p_{\mathrm{T}, i}^{\mathrm{jet}}+\sum_{j}^{N_{\mathrm{lepton}}} p_{\mathrm{T}, j}^{\ell}+E_{\mathrm{T}}^{\mathrm{miss}} .
$$

(f) $m_{b \bar{b}}$ is the invariant mass of the two leading $b$-jets in the event, and serves as a selection criterion for jet pairs to be considered as Higgs boson candidates.

(g) $m_{q \bar{q}}$ corresponds to the invariant mass of the two highest- $p_{\mathrm{T}}$ jets in the event not identified as $b$-jets. This observable, used in the $0 \ell b \bar{b}$ channel, serves as a selection criterion for jet pairs to be considered as $W$ boson candidates.

(h) $m_{\gamma \gamma}$ is the invariant mass of the two leading photons in the event, and serves as a selection criterion for photon pairs to be considered as Higgs boson candidates.

(i) $m_{\ell j(j)}$ is the invariant mass of the jet (when requiring exactly one jet), or the two leading jets system (when requiring two or more jets), and the closest lepton. The angular distance $\Delta R$ is used as the distance measure between the lepton and the jet.

(j) $m_{\ell \ell \ell}$ is the invariant mass of the three selected leptons.

(k) $m_{\mathrm{T}}$ is the transverse mass formed by the $E_{\mathrm{T}}^{\text {miss }}$ and the leading lepton in the event. It is defined as

$$
m_{\mathrm{T}}=\sqrt{2 p_{\mathrm{T}}^{\ell} E_{\mathrm{T}}^{\mathrm{miss}}\left(1-\cos \Delta \phi\left(\ell, \vec{p}_{\mathrm{T}}^{\mathrm{miss}}\right)\right)}
$$

and is used to reduce the $W+$ jets and $t \bar{t}$ backgrounds.

(1) $m_{\mathrm{T}}^{b \text {,min }}$ is the minimum transverse mass formed by $E_{\mathrm{T}}^{\mathrm{miss}}$ and up to two of the highest- $p_{\mathrm{T}} b$-jets in the event, defined as

$$
\begin{aligned}
& m_{\mathrm{T}}^{b, \min } \\
& =\min _{i \leq 2}\left(\sqrt{2 p_{\mathrm{T}}^{b \text {-jet }_{i}} E_{\mathrm{T}}^{\text {miss }}\left(1-\cos \Delta \phi\left(\vec{p}_{\mathrm{T}}^{\text {miss }}, \vec{p}_{\mathrm{T}}^{b-\text {-et }_{i}}\right)\right)}\right) .
\end{aligned}
$$

where $\min _{i \leq 2}$ selects the $b$-jet the minimizes the transverse mass.

(m) The lepton- $E_{\mathrm{T}}^{\text {miss }}-\gamma$ transverse mass $m_{\mathrm{T}}^{W \gamma_{i}}$ is calculated with respect to the $i^{\text {th }}$ photon $\gamma_{i}$, ordered in 
terms of decreasing $E_{\mathrm{T}}$, the $E_{\mathrm{T}}^{\text {miss }}$, and the identified lepton $\ell$. It is defined as

$$
\begin{aligned}
\left(m_{\mathrm{T}}^{W \gamma_{i}}\right)^{2}= & 2 E_{\mathrm{T}}^{\gamma_{i}} E_{\mathrm{T}}^{\text {miss }}\left(1-\cos \Delta \phi\left(\gamma_{i}, \vec{p}_{\mathrm{T}}^{\text {miss }}\right)\right) \\
& +2 p_{\mathrm{T}}^{\ell} E_{\mathrm{T}}^{\text {miss }}\left(1-\cos \Delta \phi\left(\ell, \vec{p}_{\mathrm{T}}^{\text {miss }}\right)\right) \\
& +2 E_{\mathrm{T}}^{\gamma_{i}} p_{\mathrm{T}}^{\ell}\left(1-\cos \Delta \phi\left(\gamma_{i}, \ell\right)\right) .
\end{aligned}
$$

(n) $m_{\mathrm{CT}}$ is the contransverse mass variable $[70,71]$ and is defined for the $b \bar{b}$ system as

$$
m_{\mathrm{CT}}=\sqrt{2 p_{\mathrm{T}}^{b_{1}} p_{\mathrm{T}}^{b_{2}}\left(1+\cos \Delta \phi_{b b}\right)},
$$

where $p_{\mathrm{T}}^{b_{1}}$ and $p_{\mathrm{T}}^{b_{2}}$ are transverse momenta of the two leading $b$-jets and $\Delta \phi_{b b}$ is the azimuthal angle between them. It is one of the main discriminating variables in selections targeting Higgs bosons decaying into $b$-quarks and is effective in suppressing the background from top-quark pair production.

(o) $m_{\mathrm{T} 2}$ is referred to as the stransverse mass and is closely related to $m_{\mathrm{T}}$. It is used to bound the masses of particles produced in pairs and each decaying into one particle that is detected and another particle that contributes to the missing transverse momentum $[72,73]$. In the case of a dilepton final state, it is defined by

$m_{\mathrm{T} 2}=\min _{q_{\mathrm{T}}}\left[\max \left(m_{\mathrm{T}}\left(\vec{p}_{\mathrm{T}}^{\ell, 1}, \vec{q}_{\mathrm{T}}\right), m_{\mathrm{T}}\left(\vec{p}_{\mathrm{T}}^{\ell, 2}, \vec{p}_{\mathrm{T}}^{\mathrm{miss}}-\vec{q}_{\mathrm{T}}\right)\right)\right]$,

where $\vec{q}_{\mathrm{T}}$ is the transverse vector that minimizes the larger of the two transverse masses $m_{\mathrm{T}}$, and $\vec{p}_{\mathrm{T}}^{\ell, 1}$ and $\vec{p}_{\mathrm{T}}^{\ell, 2}$ are the leading and subleading transverse momenta of the two leptons in the pair.

(p) The $1 \ell \gamma \gamma$ variable $\Delta \phi_{W, h}$ is the azimuthal angle between the $W$ boson and Higgs boson candidates. The $W$ boson is defined by the sum of the lepton $\vec{p}_{\mathrm{T}}^{\ell}$ and $\vec{p}_{\mathrm{T}}^{\text {miss }}$ vectors, and the Higgs boson by the sum of the transverse momentum vectors of the two photons.

\section{ANALYSIS STRATEGY}

The hadronic and leptonic decay modes of the $W$ and Higgs bosons are divided into four independent and mutually exclusive analysis channels according to key features of the visible final state: hadronic decays of both the $W$ and $h(0 \ell b \bar{b}$, Sec. VI A); hadronic $h$ decays with leptonic $W$ decays (1 $\ell b \bar{b}$, Sec. VIB); diphoton $h$ decays with leptonic $W$ decays (1 $\ell \gamma \gamma$, Sec. VIC); multilepton $h$ decays via $W, Z, \tau$ and leptonic $W$ decays $\left(\ell^{ \pm} \ell^{ \pm}\right.$and $3 \ell$, Sec. VID). Event selections and background estimation methods specific to each analysis channel are described here, as well as the signal, control, and validation region definitions (SR, CR, and VR, respectively).

The expected SM backgrounds are determined separately for each SR, and independently for each channel, with a profile likelihood fit [74], referred to as a background-only fit. The background-only fit uses the observed event yield in the associated CRs as a constraint to adjust the normalization of the dominant background processes assuming that no signal is present. The CRs are designed to be enriched in specific background contributions relevant to the analysis, while minimizing the signal contamination, and they are orthogonal to the SRs. The inputs to the background-only fit for each SR include the number of events observed in the associated $\mathrm{CR}$ and the number of events predicted by simulation in each region for all background processes. They are both described by Poisson statistics. The systematic uncertainties, described in Sec. VII, are included in the fit as nuisance parameters. They are constrained by Gaussian distributions with widths corresponding to the sizes of the uncertainties and are treated as correlated, when appropriate, between the various regions. The product of the various probability density functions forms the likelihood, which the fit maximizes by adjusting the background normalization and the nuisance parameters. Finally, the reliability of the MC extrapolation of the SM background estimates outside of the control regions is evaluated in validation regions orthogonal to CRs and SRs.

\section{A. Fully hadronic signature $(0 \ell b \bar{b})$}

\section{Event selection}

The fully hadronic analysis channel exploits the large branching ratios for both $W \rightarrow q \bar{q}$ and $h \rightarrow b \bar{b}$. Missing transverse momentum triggers are used for the trigger selection for the analysis, with an offline requirement of $E_{\mathrm{T}}^{\text {miss }}>200 \mathrm{GeV}$. Stringent event selections based on the masses of both the $W$ and Higgs boson candidates, the presence of exactly two $b$-jets, and the kinematic relationships of the final-state jets and $E_{\mathrm{T}}^{\text {miss }}$, are required in order to reduce the significant backgrounds from $t \bar{t}, Z+$ jets, $W+$ jets and single-top $W t$ production. Events are characterized by having four or five jets with $p_{\mathrm{T}}>30 \mathrm{GeV}$, exactly two of which are identified as $b$-jets, and large $m_{\mathrm{eff}}, m_{\mathrm{CT}}$, and $m_{\mathrm{T}}^{b \text {,min }}$. Two signal regions are defined, specifically targeting either high (HM) or low (LM) $\tilde{\chi}_{2}^{0}$ and $\tilde{\chi}_{1}^{ \pm}$masses (SRHad-High and SRHad-Low, respectively). The selections used are shown in Table II. The $m_{\mathrm{eff}}$ and $m_{\mathrm{T}}^{b \text {,min }}$ selections are particularly effective in reducing the $t \bar{t}$ contributions, which is the dominant background for both signal regions. The $Z+$ jets and single-top contributions are also significant, whereas the contribution from multijet production is found to be negligible and is not included. Control regions are used to constrain the normalizations of the $t \bar{t}, Z+$ jets, and $W t$ backgrounds with the data, while other processes are estimated using simulation. The $b \bar{b}$ invariant mass is required to be consistent with the Higgs boson mass, $105<m_{b \bar{b}}<135 \mathrm{GeV}$, for all signal regions. 
TABLE II. Signal region definitions for the fully hadronic $0 \ell b \bar{b}$ analysis channel.

\begin{tabular}{lcc}
\hline \hline Variable & SRHad-High & SRHad-Low \\
\hline$N_{\text {lepton }}$ & $=0$ & $=0$ \\
$N_{\text {jet }}\left(p_{\mathrm{T}}>30 \mathrm{GeV}\right)$ & $\in[4,5]$ & $\in[4,5]$ \\
$N_{b \text {-jet }}$ & $=2$ & $=2$ \\
$\Delta \phi_{\min }^{4 j}$ & $>0.4$ & $>0.4$ \\
$E_{\mathrm{T}}^{\text {miss }}[\mathrm{GeV}]$ & $>250$ & $>200$ \\
$m_{\text {eff }}[\mathrm{GeV}]$ & $>900$ & $>700$ \\
$m_{b \bar{b}}[\mathrm{GeV}]$ & $\in[105,135]$ & $\in[105,135]$ \\
$m_{q \bar{q}}[\mathrm{GeV}]$ & $\in[75,90]$ & $\in[75,90]$ \\
$m_{\mathrm{CT}}[\mathrm{GeV}]$ & $>140$ & $>190$ \\
$m_{\mathrm{T}}^{b \text { min }}[\mathrm{GeV}]$ & $>160$ & $>180$ \\
\hline \hline
\end{tabular}

All CRs and VRs select sidebands in the $m_{b \bar{b}}$ spectrum in order to remain orthogonal to the two SRs. These are further described in Sec. VI A 2.

\section{Background estimation}

The background contributions to SRHad-High and SRHad-Low are estimated using fits to the data for $t \bar{t}$, $Z+$ jets, and single-top production in specially designed control regions.

The three control regions used for estimating the $t \bar{t}$ (CRHad-TT), $Z+$ jets (CRHad-Zj), and $W t$ (CRHad-ST) contributions are further divided into high-mass (HM) and low-mass (LM) categories in order to follow the design of the SRs. These control regions are defined primarily by inverting the selections on $m_{b \bar{b}}, m_{\mathrm{CT}}, m_{\mathrm{T}}^{b \text {,min }}$, and by requiring the presence of a lepton in some cases. The $t \bar{t}$ background is estimated using $m_{\mathrm{CT}}, m_{\mathrm{T}}^{b, \min }<140 \mathrm{GeV}$ and $m_{b \bar{b}}>135 \mathrm{GeV}$ selections, while retaining the other SR requirements. This approach isolates the $t \bar{t}$ contribution while suppressing single-top and $Z+$ jets events, yielding a sample estimated to be $94 \%$ pure in $t \bar{t}$ events with negligible signal contamination. Background events from $W t$ are estimated by requiring exactly one lepton and $m_{\mathrm{CT}}>200 \mathrm{GeV}, m_{\mathrm{T}}^{b, \min }>180 \mathrm{GeV}$, and $m_{b \bar{b}}>195 \mathrm{GeV}$, and relaxing the $m_{\text {eff }}$ requirement for $\mathrm{HM}$ to $m_{\text {eff }}>700 \mathrm{GeV}$. The $Z+$ jets contribution is isolated using an opposite-sign, same-flavor, high- $p_{\mathrm{T}} 2 \ell$ requirement with $p_{\mathrm{T}, 1}^{\ell}>140 \mathrm{GeV}$ and $75<m_{\ell \ell}<105 \mathrm{GeV}$, which reduces the $t \bar{t}$ contribution to this control region. These leptons are then treated as invisible when calculating the $E_{\mathrm{T}}^{\text {miss }}$. Figure 2 shows the distribution of two key observables: the $E_{\mathrm{T}}^{\text {miss }}$ in the $t \bar{t}$ high-mass control region [Fig. 2(a)] and the $m_{b \bar{b}}$ distribution in the $Z+$ jets low-mass control region [Fig. 2(b)]. The yields estimated with the background-only fit are reported in Table III. The normalization factors are found to be $0.88 \pm 0.10(0.85 \pm 0.04)$, $1.47 \pm 0.32(1.22 \pm 0.35)$, and $0.54 \pm 0.25(0.57 \pm 0.22)$

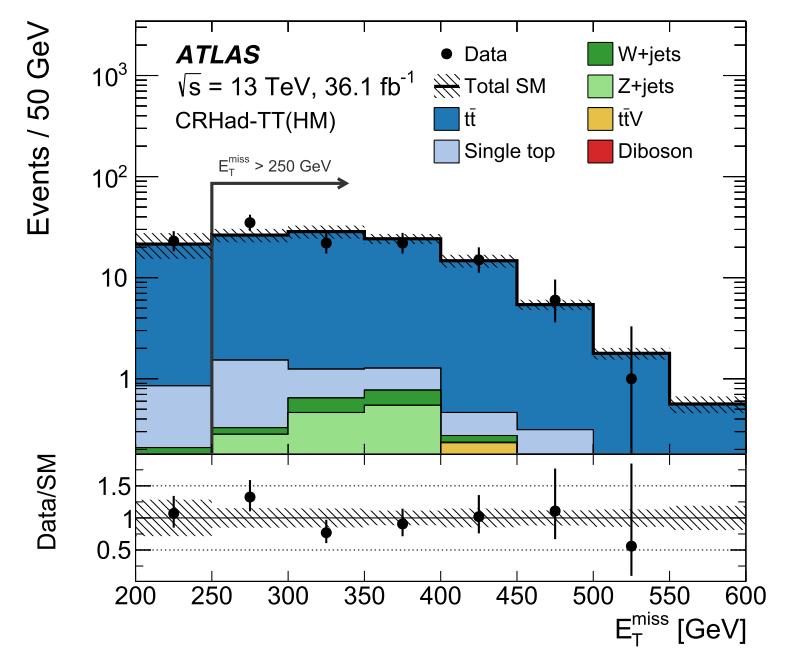

(a) $t \bar{t}$ high-mass control region

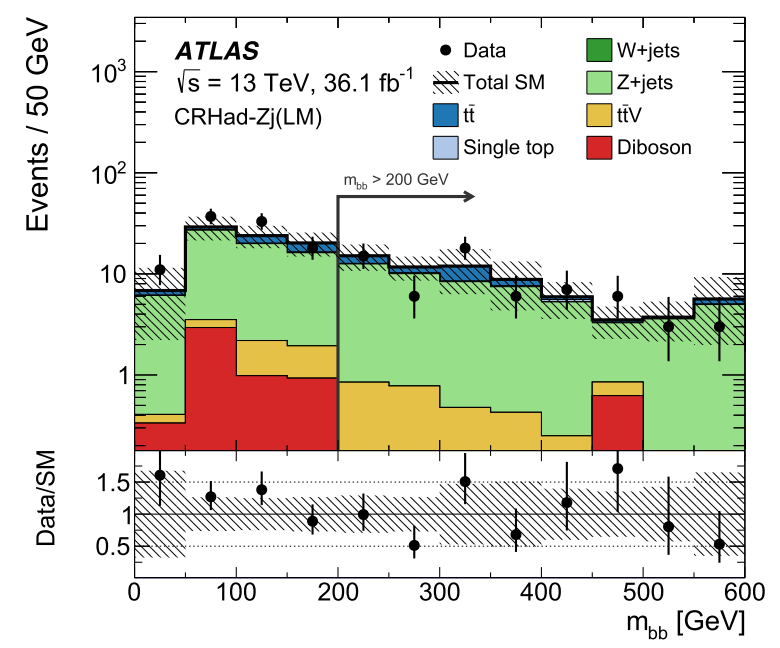

(b) $Z+$ jets low-mass control region

FIG. 2. Comparisons of data with SM predictions in $t \bar{t}$ and $Z+$ jets control regions for representative kinematic distributions: (a) $E_{\mathrm{T}}^{\text {miss }}$ for the $t \bar{t}$ high-mass control region and (b) $m_{b \bar{b}}$ for the $Z+$ jets low-mass control region. Predictions from simulation are shown after the background-only fit. The arrow indicates the selection on that variable used to define the corresponding CRs. The uncertainty bands include statistical and systematic uncertainties.

for $t \bar{t}, Z+$ jets, and $W t$ in the high-mass (low-mass) signal region, respectively. The errors include statistical and systematic uncertainties. No diboson MC simulation events are found to contribute to the CRHad-ST regions.

To validate the background prediction, three sets of validation regions are defined so as to be similar, but orthogonal, to the SRs. The $t \bar{t}$ VRs for each SR (VRHadTT, for HM or LM) reverse the $m_{\mathrm{CT}}$ selections, requiring $m_{\mathrm{CT}}<140$ (190) GeV for HM (LM), select the sideband $m_{b \bar{b}}>135 \mathrm{GeV}$ (orthogonal to the SRs), but retain the SR selection on $m_{\mathrm{T}}^{b, \min }$. In order to validate the $W t$ and $Z+$ jets 
TABLE III. Fit results in the control regions for the $0 \ell b \bar{b}$ channel. The results are obtained from the control regions using the background-only fit. The errors shown are the statistical plus systematic uncertainties. Uncertainties in the fitted yields are symmetric by construction, where the negative error is truncated when reaching zero event yield.

\begin{tabular}{lcccccc}
\hline \hline CR channels & CRHad-TT(HM) & CRHad-ST(HM) & CRHad-Zj(HM) & CRHad-TT(LM) & CRHad-ST(LM) & CRHad-Zj(LM) \\
\hline Observed events & 102 & 17 & 39 & 695 & 23 & 78 \\
Fitted background events & $102 \pm 10$ & $17 \pm 4$ & $39 \pm 6$ & $695 \pm 26$ & $23 \pm 5$ & $78 \pm 9$ \\
$t \bar{t}$ & $97 \pm 11$ & $3.7 \pm 2.0$ & $2.9 \pm 2.4$ & $659 \pm 34$ & $4.7 \pm 2.3$ & $10_{-10}^{+12}$ \\
Single top & $2.7_{-2.7}^{+3.5}$ & $10 \pm 5$ & $0.8_{-0.8}^{+0.9}$ & $19 \pm 19$ & $15 \pm 6$ & $1.0 \pm 0.9$ \\
$W+$ jets & $0.5_{-0.5}^{+0.6}$ & $2.2 \pm 1.1$ & $0.0059 \pm 0.0025$ & $3.9 \pm 3.1$ & $2.8 \pm 1.2$ & $0.0059 \pm 0.0026$ \\
$Z+$ jets & $1.1 \pm 0.6$ & $0.08 \pm 0.07$ & $32 \pm 7$ & $9.5 \pm 3.2$ & $0.09 \pm 0.04$ & $63 \pm 17$ \\
$t \bar{t}+V$ & $0.63 \pm 0.14$ & $0.62 \pm 0.16$ & $2.0 \pm 0.4$ & $3.1 \pm 0.5$ & $0.80 \pm 0.17$ & $3.7 \pm 0.6$ \\
Diboson & $0.08_{-0.08}^{+0.14}$ & $<0.07$ & $0.8 \pm 0.8$ & $1.16 \pm 0.34$ & $<0.07$ & $0.8 \pm 0.5$ \\
\hline \hline
\end{tabular}

estimates, VRs are defined using sideband regions in the $m_{b \bar{b}}$ and $m_{q \bar{q}}$ spectra, either by vetoing the SR range in both of these variables, $m_{b \bar{b}} \notin[105,135] \mathrm{GeV}$ and $m_{q \bar{q}} \notin[75,90] \mathrm{GeV}$ (VRHad-SB for HM and LM), or by selecting the $m_{b \bar{b}}>135 \mathrm{GeV}$ sideband and imposing a $W$ mass requirement on the non- $b$-tagged dijet invariant mass, $75<m_{q \bar{q}}<90 \mathrm{GeV}$ (VRHad-bbhigh, for HM or LM).

The number of events predicted by the background-only fit is compared with the data in the VRs in the upper panel of Fig. 3. The pull, defined by the difference between the observed number of events $\left(n_{\mathrm{obs}}\right)$ and the predicted background yield $\left(n_{\text {pred }}\right)$ divided by the total uncertainty $\left(\sigma_{\text {tot }}\right)$, is shown for each region in the lower panel. No evidence of significant background mismodeling is observed in the VRs.

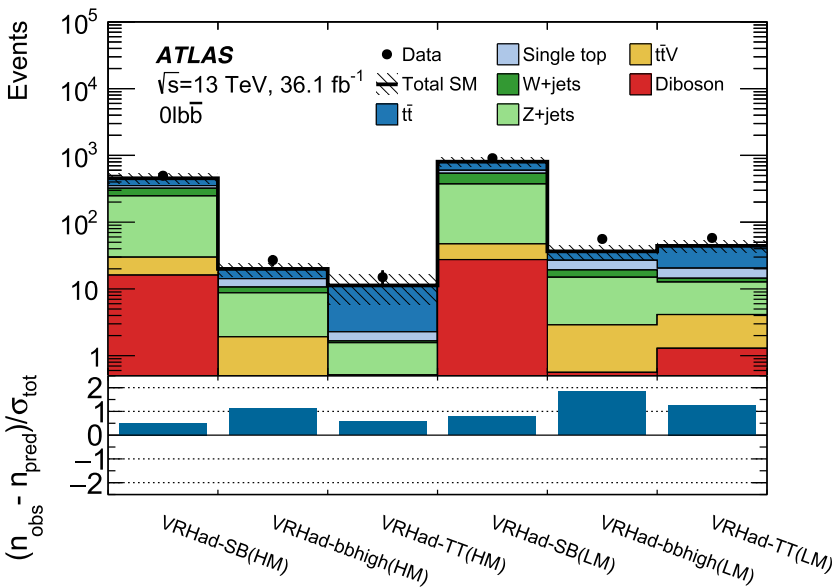

FIG. 3. Comparison of the predicted backgrounds with the observed numbers of events in the VRs associated with the $0 \ell b \bar{b}$ channel. The normalization of the backgrounds is obtained from the fit to the CRs. The upper panel shows the observed number of events and the predicted background yield. All uncertainties are included in the uncertainty band. The lower panel shows the pulls in each VR.

\section{B. Single-lepton plus di- $b$-jet signature $(1 \ell b \bar{b})$}

\section{Event selection}

The events considered in the one-lepton plus two- $b$-jets channel are also recorded with the $E_{\mathrm{T}}^{\text {miss }}$ trigger, with an offline requirement of $E_{\mathrm{T}}^{\text {miss }}>200 \mathrm{GeV}$. Events with exactly one electron or muon are selected if they also contain two or three jets with $p_{\mathrm{T}}>25 \mathrm{GeV}$, two of which must be $b$-tagged. Discriminating variables are used to separate the signal from backgrounds, and include $E_{\mathrm{T}}^{\text {miss }}$, $m_{\mathrm{T}}$, the invariant mass of the two $b$-jets and their contransverse mass. The dominant SM background contributions in the $1 \ell b \bar{b}$ channel are $t \bar{t}, W+$ jets, and single-top ( $W t$ channel) production. Three sets of signal regions are defined and optimized to target different LSP and nextto-lightest neutralino or chargino mass hierarchies. The three regions, labeled as SR1Lbb-Low, SR1Lbb-Medium, and SR1Lbb-High, are summarized in Table IV. SR1LbbLow provides sensitivity to signal models with a masssplitting between LSP and next-to-lightest neutralino similar to the Higgs boson mass, while SR1Lbb-Medium and -High target mass-splittings between 150 and $250 \mathrm{GeV}$ and above $250 \mathrm{GeV}$, respectively. The $m_{\mathrm{CT}}$ distribution of

TABLE IV. Summary of the event selection for signal regions of the $1 \ell b \bar{b}$ channel.

\begin{tabular}{lccc}
\hline \hline Variable & $\begin{array}{c}\text { SR1Lbb- } \\
\text { Low }\end{array}$ & $\begin{array}{c}\text { SR1Lbb- } \\
\text { Medium }\end{array}$ & $\begin{array}{c}\text { SR1Lbb- } \\
\text { High }\end{array}$ \\
\hline$N_{\text {lepton }}$ & & $=1$ & \\
$p_{\mathrm{T}}^{\ell}[\mathrm{GeV}]$ & & $>27$ & \\
$N_{\text {jet }}\left(p_{\mathrm{T}}>25 \mathrm{GeV}\right)$ & & $=2$ or 3 & \\
$N_{b \text {-jet }}$ & & $=2$ & \\
$E_{\mathrm{T}}^{\text {miss }}[\mathrm{GeV}]$ & & $>200$ & \\
$m_{\mathrm{CT}}[\mathrm{GeV}]$ & & $>160$ & \\
$m_{\mathrm{T}}[\mathrm{GeV}]$ & $\in[100,140]$ & $\in[140,200]$ & $>200$ \\
$m_{b \bar{b}}[\mathrm{GeV}]$ & & $\in[105,135]$ & \\
\hline \hline
\end{tabular}


TABLE V. Fit results in the control regions for the $1 \ell b \bar{b}$ channel. The results are obtained from the control regions using the background-only fit. The category "Others" includes contributions from $W h$ production and $Z+$ jets. The errors shown are the statistical plus systematic uncertainties. Uncertainties in the fitted yields are symmetric by construction, where the negative error is truncated when reaching zero event yield.

\begin{tabular}{|c|c|c|c|c|c|}
\hline CR channels & CR1Lbb-TT(LM) & CR1Lbb-TT(MM) & CR1Lbb-TT(HM) & CR1Lbb-Wj & CR1Lbb-ST \\
\hline Observed events & 192 & 359 & 1115 & 72 & 65 \\
\hline Fitted background events & $192 \pm 14$ & $359 \pm 19$ & $1115 \pm 34$ & $72 \pm 9$ & $65 \pm 8$ \\
\hline$t \bar{t}$ & $147 \pm 33$ & $325 \pm 32$ & $1020 \pm 90$ & $15 \pm 14$ & $20_{-20}^{+23}$ \\
\hline Single top & $28 \pm 25$ & $22_{-22}^{+24}$ & $60_{-60}^{+70}$ & $4_{-4}^{+6}$ & $33 \pm 25$ \\
\hline$W+$ jets & $16 \pm 7$ & $7.3 \pm 2.7$ & $25 \pm 11$ & $51 \pm 17$ & $8 \pm 4$ \\
\hline$t \bar{t}+V$ & $1.16 \pm 0.20$ & $2.8 \pm 0.4$ & $6.9 \pm 1.1$ & $0.079 \pm 0.022$ & $3.2 \pm 0.6$ \\
\hline Diboson & $0.57 \pm 0.24$ & $0.92 \pm 0.29$ & $1.3 \pm 0.4$ & $2.1 \pm 1.1$ & $0.84 \pm 0.28$ \\
\hline Others & $0.125 \pm 0.032$ & $0.20 \pm 0.06$ & $1.9 \pm 0.5$ & $0.24 \pm 0.17$ & $0.10 \pm 0.04$ \\
\hline
\end{tabular}

the $t \bar{t}$ background has an upper endpoint approximately equal to the top-quark mass, and thus this background is efficiently suppressed by requiring $m_{\mathrm{CT}}>160 \mathrm{GeV}$ in all regions. The $W+$ jets background is reduced by selecting events with $m_{\mathrm{T}}>100 \mathrm{GeV}$. The three SRs require $100<m_{\mathrm{T}}<140 \mathrm{GeV}, 140<m_{\mathrm{T}}<200 \mathrm{GeV}$, and $m_{\mathrm{T}}>$ $200 \mathrm{GeV}$ for SR1Lbb-Low, -Medium and -High, respectively. Finally, the $b \bar{b}$ invariant mass is required to be $105<m_{b \bar{b}}<135 \mathrm{GeV}$, consistent with the Higgs boson mass, for all regions.

\section{Background estimation}

The contributions from the $t \bar{t}, W t$, and $W+$ jets background sources are estimated from MC simulation, but with yields that are normalized to data in dedicated CRs. The contribution from multijet production, where the lepton is misidentified as a jet or originates from a heavy-flavor hadron decay or photon conversion, is found to be negligible and neglected in the following. The remaining sources of background (single-top $t$ - and $s$-channels, $Z+$ jets, diboson, $Z h$, and $W h$ production), including their total yields, are estimated from simulation.

Three sets of CRs, CR1Lbb-TT, CR1Lbb-ST and CR1Lbb- $\mathrm{Wj}$, are designed to estimate the $t \bar{t}, W t$, and $W+$ jets background processes, respectively. The acceptance for $t \bar{t}$ events is increased in CR1Lbb-TT by requiring $m_{\mathrm{CT}}<$ $160 \mathrm{GeV}$ and inverting the selection on $m_{b \bar{b}}$. Three $t \bar{t} \mathrm{CRs}$ are defined as a function of $m_{\mathrm{T}}$ mirroring the Low, Medium and High SR selections. Contributions from $W+$ jets events are estimated using a common CR1Lbb-Wj for all SRs, where events are required to have $40<m_{\mathrm{T}}<$ $100 \mathrm{GeV}$ and $m_{b \bar{b}}<80 \mathrm{GeV}$. CR1Lbb-ST is designed to be orthogonal to the three CR1Lbb-TTs and CR1Lbb-Wj by requiring events to have $m_{\mathrm{CT}}>160 \mathrm{GeV}, m_{b \bar{b}}>$ $195 \mathrm{GeV}$ and $m_{\mathrm{T}}>100 \mathrm{GeV}$.

The yields estimated with the background-only fit are reported in Table V. The normalization factors are found to be between $0.89_{-0.20}^{+0.21}$ and $1.15 \pm 0.13$ for the three SRs' $t \bar{t}$ estimates, $1.1_{-1.1}^{+0.7}$ for $W t$ and $1.4 \pm 0.5$ for $W+$ jets, where the errors include statistical and systematic uncertainties. Figure 4 shows representative comparisons of data with MC simulation for $m_{b \bar{b}}, m_{\mathrm{T}}$ and $E_{\mathrm{T}}^{\text {miss }}$ distributions in $t \bar{t}$, $W+$ jets and single-top control regions. The data agree well with the SM predictions in all distributions.

To validate the background predictions, two sets of VRs are defined similarly but orthogonal to the SRs. VR1Lbb-onpeak regions are defined similarly to the three CR1Lbb-TT regions but requiring $105<m_{b \bar{b}}<135 \mathrm{GeV}$. VR1Lbb-offpeak requires $m_{\mathrm{CT}}>160 \mathrm{GeV}, m_{b \bar{b}}$ below $95 \mathrm{GeV}$ or in the range $145-195 \mathrm{GeV}$ and $E_{\mathrm{T}}^{\text {miss }}>$ $180 \mathrm{GeV}$. The yields and pulls in each VR are shown in Fig. 5 after the background-only fit. The data event yields are found to be consistent with background expectations.

\section{Single-lepton plus diphoton signature (1 $\ell \gamma \gamma)$}

\section{Event selection}

Events used in the single-lepton plus diphoton ( $1 \ell \gamma \gamma)$ channel were recorded with a diphoton trigger using a trigger-level requirement of $E_{\mathrm{T}}>35 \mathrm{GeV}$ and $E_{\mathrm{T}}>$ $25 \mathrm{GeV}$ for the leading and subleading photons, respectively. For these events, the selection requires exactly one lepton ( $e$ or $\mu$ ) with $p_{\mathrm{T}}>25 \mathrm{GeV}$ and exactly two photons. To achieve full trigger efficiency, the leading and subleading photons are required to have a minimum $E_{\mathrm{T}}$ of $40 \mathrm{GeV}$ and $31 \mathrm{GeV}$, respectively. The diphoton invariant mass $m_{\gamma \gamma}$, which is measured in the region of the Higgs boson mass with a resolution of approximately $1.7 \mathrm{GeV}$, is required to lie within the mass window $120<m_{\gamma \gamma}<130 \mathrm{GeV}$. This effectively rejects SM backgrounds without a Higgs boson in the final state, referred to as nonpeaking backgrounds. These backgrounds, which include SM diphoton and $V \gamma \gamma$ $(V=W, Z)$ production, vary slowly across the selected mass window and can be reliably estimated from sidebands above and below the narrow mass window assuming a flat distribution. Observables such as $E_{\mathrm{T}}^{\text {miss }}, m_{\mathrm{T}}, m_{\mathrm{T}}^{W \gamma_{1}}, m_{\mathrm{T}}^{W \gamma_{2}}$, $\Delta \phi_{W, h}$ and the number of $b$-jets provide additional discrimination between signal and both the peaking 


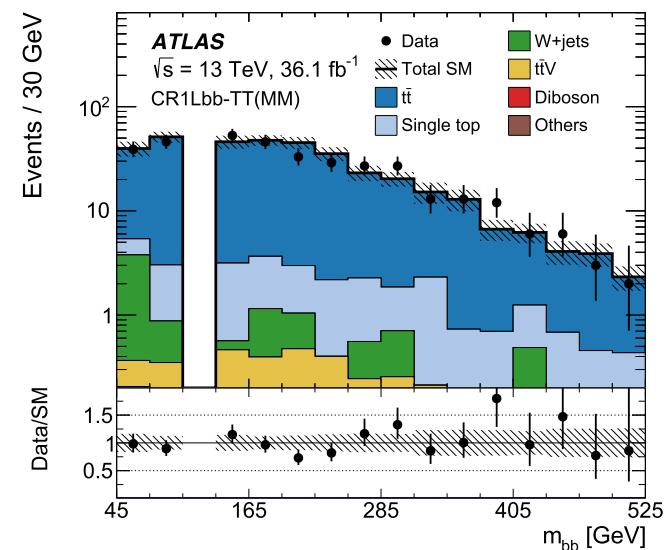

(a) $m_{b \bar{b}}$ for $t \bar{t}$ medium-mass (MM) control region

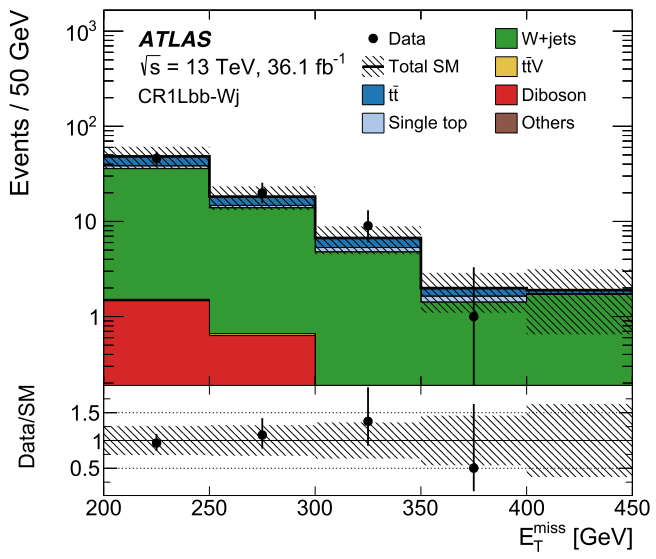

(c) $E_{\mathrm{T}}^{\text {miss }}$ for $W+$ jets control region

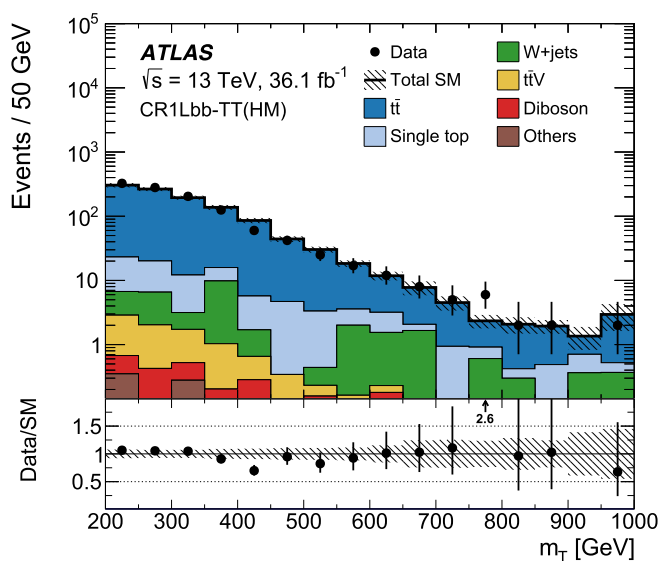

(b) $m_{\mathrm{T}}$ for $t \bar{t}$ high-mass (HM) control region

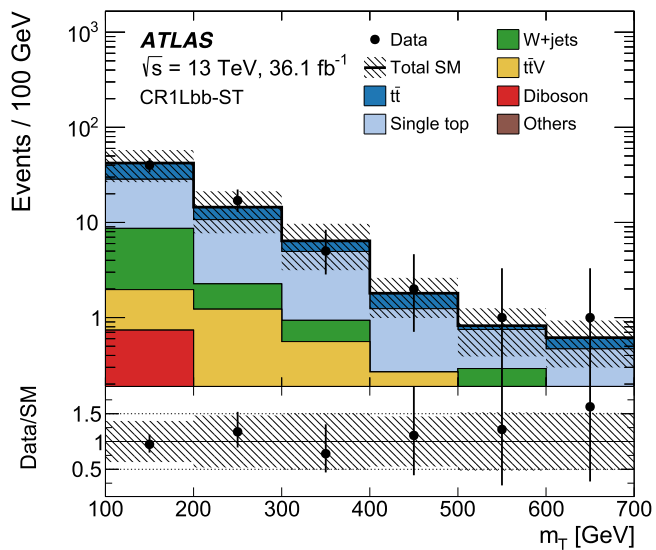

(d) $m_{\mathrm{T}}$ for single-top control region

FIG. 4. Comparison of data with $\mathrm{SM}$ predictions in $t \bar{t}, W+$ jets, and single-top control regions for representative kinematic distributions: (a) $m_{b \bar{b}}$ for CR1Lbb-TT medium, (b) $m_{\mathrm{T}}$ for CR1Lbb-TT high, (c) $E_{\mathrm{T}}^{\text {miss }}$ for CR1Lbb-Wj, and (d) $m_{\mathrm{T}}$ for CR1Lbb-ST. Predictions from MC simulation are shown after the background-only fit. The uncertainty bands include statistical and systematic uncertainties.

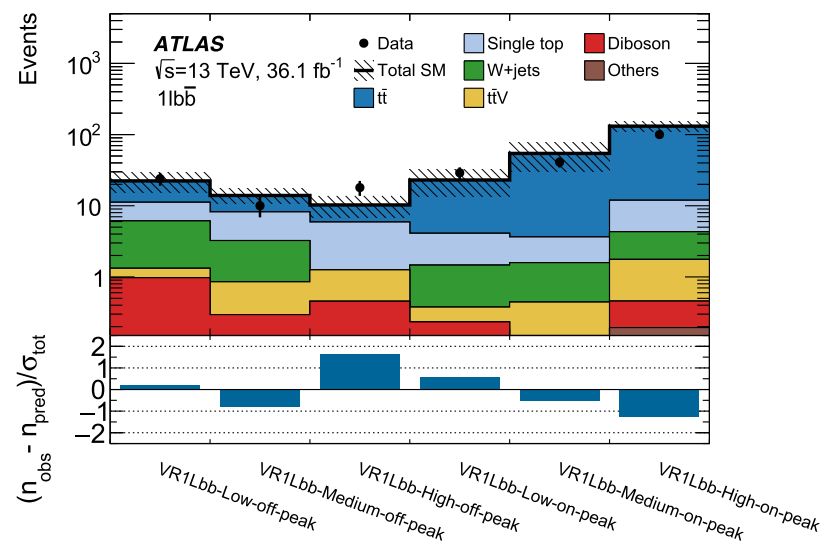

FIG. 5. Comparison of the predicted backgrounds with the observed numbers of events in the validation regions associated with the $1 \ell b \bar{b}$ channel. The normalization of the backgrounds is obtained from the fit to the CRs. The upper panel shows the observed number of events and the predicted background yield. All uncertainties are included in the uncertainty band. The lower panel shows the pulls in each VR. backgrounds (containing a Higgs boson decaying into two photons) and the nonpeaking backgrounds.

The dominant peaking background arises from Wh production, which can be difficult to distinguish from the signal, which itself includes both a $W$ and a Higgs boson. After applying a series of selection criteria optimized to separate signal from both the peaking and nonpeaking backgrounds (see Table VI), the resulting inclusive $\mathrm{SR}$ is subdivided into a region largely depleted of $W h$ backgrounds (SR1L $\gamma \gamma$-a) and a SR with a significant contribution from $W h$ production (SR1L $\gamma \gamma-\mathrm{b})$.

\section{Background estimation}

Nonpeaking backgrounds are estimated separately for SR1L $\gamma \gamma$-a and SR1L $\gamma \gamma$-b by measuring the event yields, per $10 \mathrm{GeV}$ in $m_{\gamma \gamma}$, in the lower and upper sidebands within $105<m_{\gamma \gamma}<120 \mathrm{GeV}$ and $130<m_{\gamma \gamma}<160 \mathrm{GeV}$, respectively. The yields are determined by fitting a constant function to the observed events in sidebands. Results 
TABLE VI. Summary of the event selection for the two regions of the $1 \ell \gamma \gamma$ channel, SR1L $\gamma \gamma-\mathrm{a}$ and SR1L $\gamma \gamma-\mathrm{b}$.

\begin{tabular}{|c|c|c|c|}
\hline Variable & $\mathrm{SR} 1 \mathrm{~L} \gamma \gamma-\mathrm{a}$ & & SR1L $\gamma \gamma-b$ \\
\hline$N_{\gamma}$ & & $=2$ & \\
\hline$p_{\mathrm{T}}^{\gamma}[\mathrm{GeV}]$ & & $>(40,31)$ & \\
\hline$N_{\text {lepton }}$ & & $=1$ & \\
\hline$p_{\mathrm{T}}^{\ell}[\mathrm{GeV}]$ & & $>25$ & \\
\hline$E_{\mathrm{T}}^{\text {miss }}[\mathrm{GeV}]$ & & $>40$ & \\
\hline$\Delta \phi_{W, h}$ & & $>2.25$ & \\
\hline$m_{\gamma \gamma}[\mathrm{GeV}]$ & & $\in[120,130]$ & \\
\hline$N_{b \text {-jet }}\left(p_{\mathrm{T}}>30 \mathrm{GeV}\right)$ & & $=0$ & \\
\hline$m_{\mathrm{T}}^{W \gamma_{1}}[\mathrm{GeV}]$ & & $\geq 150$ & \\
\hline$m_{\mathrm{T}}^{W \gamma_{2}}[\mathrm{GeV}]$ & $>140$ & & $\in[80,140]$ \\
\hline$m_{\mathrm{T}}[\mathrm{GeV}]$ & $>110$ & & $<110$ \\
\hline
\end{tabular}

obtained by fitting a linear function are found to be consistent. The observation of 1 (15) event(s) in the sidebands around SR1L $\gamma \gamma-\mathrm{a}(\mathrm{SR} 1 \mathrm{~L} \gamma \gamma-\mathrm{b})$ leads to an expectation of $0.22 \pm 0.22(3.3 \pm 0.9)$ nonpeaking background events, with the uncertainty dominated by the number of events in the sideband data sample.

Peaking backgrounds are estimated from MC simulations of the production of the Higgs boson through gluon-gluon and vector-boson fusion, and of Higgs boson production in association with a $W$ or $Z$ boson. Production of a Higgs boson in association with a $t \bar{t}$ pair is also taken into account, although this contribution is suppressed by the requirement that the events contain no $b$-jets. A value of $(2.28 \pm 0.08) \times$ $10^{-3}$ is assumed for the $h \rightarrow \gamma \gamma$ branching ratio [75]. Production of $W h$ events, with a subsequent decay of the Higgs boson into two photons, is expected to account for approximately $90 \%$ of the peaking background in the two SRs. Altogether, a total of $0.14 \pm 0.02(2.01 \pm 0.30)$ events are expected to arise from peaking backgrounds in SR1L $\gamma \gamma$-a (SR1L $\gamma \gamma$-b).

\section{Same-sign dilepton and three-lepton signatures $\left(\boldsymbol{\ell}^{ \pm} \boldsymbol{\ell}^{ \pm}, 3 \boldsymbol{\ell}\right)$}

Two- or three-lepton (multilepton) signatures arise when the $W$ boson produced in conjunction with the Higgs boson decays semileptonically and the Higgs boson itself decays into one of $W W, Z Z$ or $\tau \tau$, and these in turn yield at least one other lepton in the final state. Final-state neutrinos and lightest neutralinos all contribute to sizable $E_{\mathrm{T}}^{\text {miss }}$ in multileptonic signal events. Two sets of signal regions, kinematically orthogonal, are defined according to the presence of either exactly two leptons with same-sign electric charge $\left(\ell^{ \pm} \ell^{ \pm}\right.$analysis), or exactly three leptons satisfying various requirements on lepton-flavor and electric-charge combinations ( $3 \ell$ analysis). The $\ell^{ \pm} \ell^{ \pm}$and $3 \ell$ analyses share the same trigger. Events must pass a trigger selection that combines single- and two-lepton triggers into a logical OR, where trigger thresholds on lepton $p_{\mathrm{T}}$ between 8 and $140 \mathrm{GeV}$ are applied in conjunction with trigger-specific lepton identification criteria. Selected leptons have offline requirements of $p_{\mathrm{T}}>25 \mathrm{GeV}$ to ensure that trigger efficiencies are maximal and uniform in the relevant phase space. For both analyses, events with additional leptons are removed, and a $b$-jet veto is applied such that there are zero $b$-jets with $p_{\mathrm{T}}>20 \mathrm{GeV}$. Non- $b$-tagged jets are not vetoed, and are required in some signal regions to account for hadronic decays of intermediate particles (e.g. $W$ bosons), or for the presence of initial-state radiation. Jets in both the $\ell^{ \pm} \ell^{ \pm}$and $3 \ell$ signal regions are required to have $p_{\mathrm{T}}>20 \mathrm{GeV}$ and pass the quality and kinematic selections described in Sec. IV. The signal region optimization and background estimations are developed independently for $\ell^{ \pm} \ell^{ \pm}$and $3 \ell$ events.

Two primary sources of background are distinguished in these analyses. The first category is the reducible background, which includes events containing at least one fake or nonprompt (FNP) lepton (referred to as fake background) and, for the $\ell^{ \pm} \ell^{ \pm}$analysis only, events containing electrons with misidentified charge (referred to as charge-flip background). This background primarily arises from the production of top-quark pairs. The FNP lepton typically originates from heavy-flavor hadron decays in events containing top quarks, or $W$ or $Z$ bosons. Those are suppressed for the $\ell^{ \pm} \ell^{ \pm}$and $3 \ell$ analyses by vetoing $b$-tagged jets, while hadrons misidentified as leptons, electrons from photon conversions, and leptons from pion or kaon decays in flight remain as other possible sources. Data-driven methods are used for the estimation of this reducible background in the signal and validation regions. The second background category is the irreducible background from events with two same-sign prompt leptons or at least three prompt leptons. It is estimated using simulation samples and is dominated by diboson ( $W Z$ and $Z Z$ ) production. Dedicated validation regions with enhanced contributions from these processes, and small signal contamination, are defined to verify the background predictions from the simulation.

Details of the estimates of both the reducible and irreducible backgrounds for each analysis are given in the following subsections.

\section{1. $\boldsymbol{\ell}^{ \pm} \boldsymbol{\ell}^{ \pm}$event selection and background estimation}

Two signal regions are defined for the $\ell^{ \pm} \ell^{ \pm}$analysis channel, requiring either exactly one jet (SRSS-j1) or two to three (SRSS-j23) jets. In both regions, events must satisfy $E_{\mathrm{T}}^{\text {miss }}>100 \mathrm{GeV}$, while region-specific requirements are applied on the transverse mass $m_{\mathrm{T}}$, the effective mass $m_{\text {eff }}$, the stransverse mass $m_{\mathrm{T} 2}$, and the kinematic variable $m_{\ell j(j)}$, which in signal events provides an estimate of the visible mass of the Higgs boson candidate. The $\ell^{ \pm} \ell^{ \pm}$ signal region selections are summarized in Table VII. 


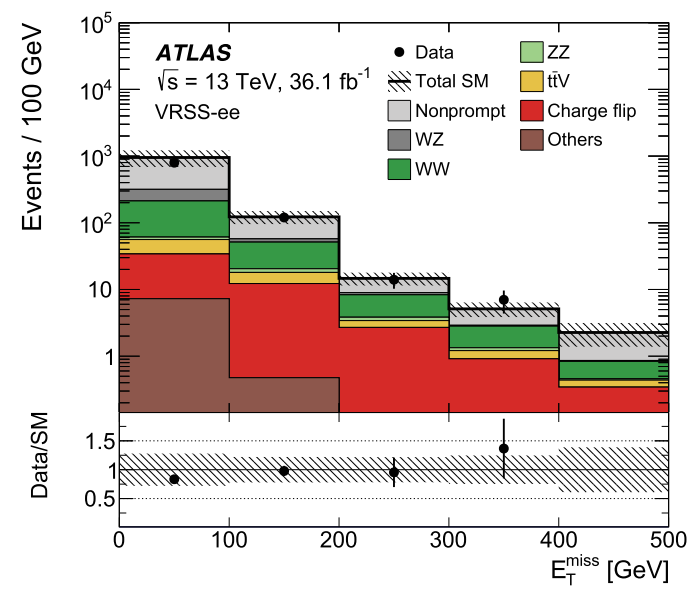

(a) $E_{\mathrm{T}}^{\text {miss }}$ in $\ell^{ \pm} \ell^{ \pm}$VRSS-ee validation region

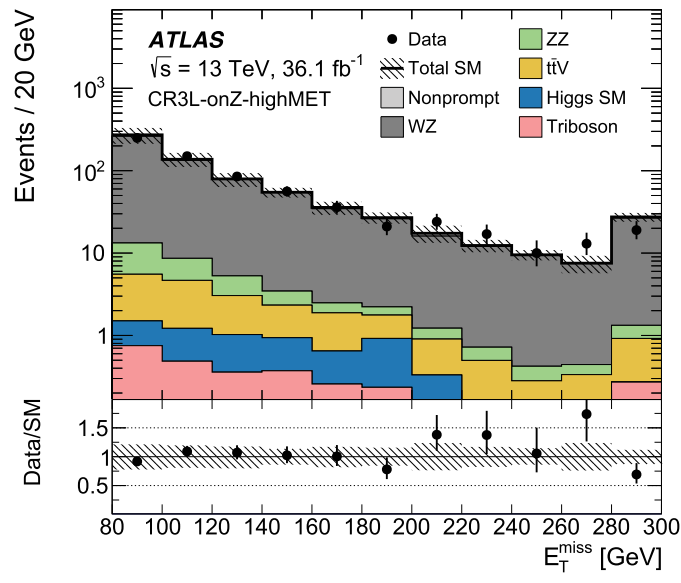

(b) $E_{\mathrm{T}}^{\text {miss }}$ in $3 \ell Z$-boson control

FIG. 6. (a) $E_{\mathrm{T}}^{\text {miss }}$ distribution in the electron-type VRSS-ee for $\ell^{ \pm} \ell^{ \pm}$channel (used to estimate FNP leptons background) and (b) $E_{\mathrm{T}}^{\text {miss }}$ distribution in the on-shell $Z$-boson $\mathrm{CR}$ for $3 \ell$ (used to estimate the $W Z$ normalization).

The reducible FNP background is estimated using the matrix method [76,77]. The matrix method uses both relaxed and more stringent lepton identification criteria in order to isolate the contributions from FNP leptons in a given data sample. The two sets of identification criteria that are used are referred to as tight and loose. The matrix method relates the number of events containing prompt or FNP leptons to the number of observed events with tight or loose-but-not-tight leptons using the probability, $\mathcal{O}\left(10^{-1}-10^{-2}\right)$, for loose prompt or FNP leptons to satisfy the tight criteria. Inputs to the method are the probability for loose prompt leptons to satisfy the tight selection criteria, estimated using $Z \rightarrow \ell \ell$ events, and the probability for loose FNP leptons to satisfy the tight selection criteria, determined from data in SS control regions enriched in nonprompt leptons. Final yields for FNP backgrounds are validated in VRs. Figure 6(a) shows the $E_{\mathrm{T}}^{\text {miss }}$ distribution in the VR for the $\ell^{ \pm} \ell^{ \pm}$channel in the case of electrons (VRSS-ee) and good agreement is found between data and predictions.

Charge misidentification is only relevant for electrons and the contribution of charge-flip events to the SRs and

TABLE VII. Summary of the event selections for the $\ell^{ \pm} \ell^{ \pm}$ signal regions.

\begin{tabular}{lcc}
\hline \hline Variable & SRSS-j1 & SRSS-j23 \\
\hline$\Delta \eta_{\ell \ell}$ & $<1.5$ & $\ldots$ \\
$N_{\text {jet }}\left(p_{\mathrm{T}}>20 \mathrm{GeV}\right)$ & $=1$ & $=2$ or 3 \\
$N_{b \text {-jet }}$ & $=0$ & $=0$ \\
$E_{\mathrm{T}}^{\text {miss }}[\mathrm{GeV}]$ & $>100$ & $>100$ \\
$m_{\mathrm{T}}[\mathrm{GeV}]$ & $>140$ & $>120$ \\
$m_{\mathrm{eff}}[\mathrm{GeV}]$ & $>260$ & $>240$ \\
$m_{\ell j(j)}[\mathrm{GeV}]$ & $<180$ & $<130$ \\
$m_{\mathrm{T} 2}[\mathrm{GeV}]$ & $>80$ & $>70$ \\
\hline \hline
\end{tabular}

VRs is estimated using the data. The electron charge-flip probability is extracted in a $Z \rightarrow e e$ data sample using a likelihood fit which takes as input the numbers of samesign and opposite-sign electron pairs observed in a 80$100 \mathrm{GeV}$ electron-pair mass window. It is treated as a free parameter of the fit and it is found to be between $2 \times 10^{-4}$ and $10^{-3}$ depending on the $p_{\mathrm{T}}$ and $\eta$ of the electron. Sources of SM irreducible background arise from $W Z$ and $\mathrm{ZZ}$ events and are evaluated using simulation.

The background estimates are validated in dedicated VRs defined for each signal region and referred to as VRSS- 1 and VRSS-j23. In VRSS- 1 1, events are required to pass all selections as in SRSS-j1 but for $E_{\mathrm{T}}^{\text {miss }}$, required to be between $70 \mathrm{GeV}$ and $100 \mathrm{GeV}$, and $m_{\ell j(j)}>130 \mathrm{GeV}$.

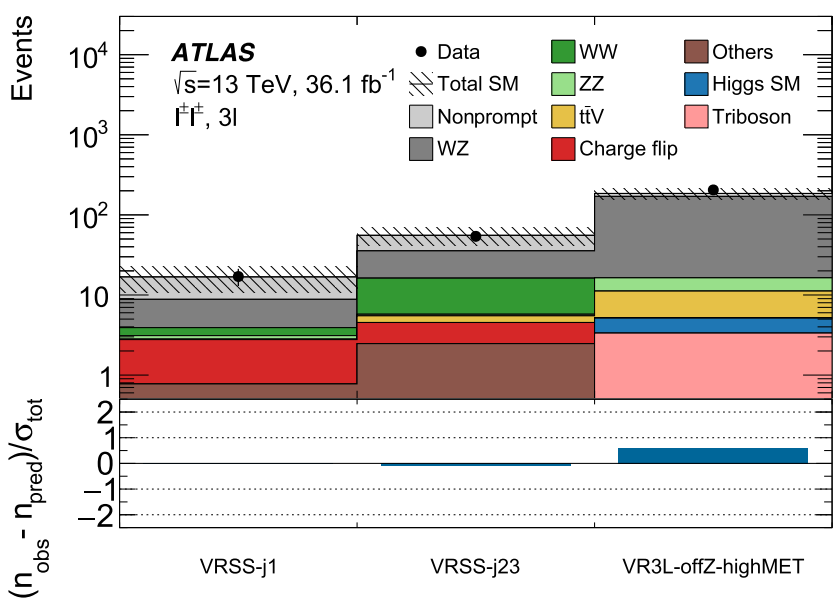

FIG. 7. Results of the likelihood fit extrapolated to the VRs associated with both the $\ell^{ \pm} \ell^{ \pm}$and $3 \ell$ channels. The normalization of the backgrounds is obtained from the fit to the CRs. The upper panel shows the observed number of events and the predicted background yield. All uncertainties are included in the uncertainty band. The lower panel shows the pulls in each VR. 
TABLE VIII. Summary of the event selection for DFOS $3 \ell$ signal regions.

\begin{tabular}{lccc}
\hline \hline Variable & SR3L-DFOS-0J & SR3L-DFOS-1Ja & SR3L-DFOS-1Jb \\
\hline$N_{\text {jet }}\left(p_{\mathrm{T}}>20 \mathrm{GeV}\right)$ & $=0$ & $>0$ & $>0$ \\
$N_{b \text {-jet }}$ & $=0$ & $=0$ & $=0$ \\
$E_{\mathrm{T}}^{\text {miss }}[\mathrm{GeV}]$ & $>60$ & $\in[30,100]$ & $>100$ \\
$m_{\ell_{\text {DFos }}+\ell_{\text {near }}}[\mathrm{GeV}]$ & $<90$ & $<60$ & $<70$ \\
$\Delta R_{\text {OS near }}$ & $\cdots$ & $<1.4$ & $<1.4$ \\
$\Delta \phi_{\mathrm{SS}}$ & $\cdots$ & $\cdots$ & $<2.8$ \\
\hline \hline
\end{tabular}

TABLE IX. Summary of the event selection for SFOS $3 \ell$ signal regions.

\begin{tabular}{lccc}
\hline \hline Variable & SR3L-SFOS-0Ja & SR3L-SFOS-0Jb & SR3L-SFOS-1J \\
\hline$N_{\text {jet }}\left(p_{\mathrm{T}}>20 \mathrm{GeV}\right)$ & $=0$ & $=0$ & $>0$ \\
$N_{b \text {-jet }}$ & $=0$ & $=0$ & $=0$ \\
$E_{\mathrm{T}}^{\operatorname{miss}}[\mathrm{GeV}]$ & $\in[80,120]$ & $>120$ & $>110$ \\
$m_{\mathrm{T}}{ }_{\min }[\mathrm{GeV}]$ & $>110$ & $>110$ & $>110$ \\
$m_{\mathrm{SFOS}}^{\min }$ & $>20 \mathrm{GeV}, \notin[81.2,101.2]$ & $>20 \mathrm{GeV}, \notin[81.2,101.2]$ & $>20 \mathrm{GeV}, \notin[81.2,101.2]$ \\
\hline \hline
\end{tabular}

No selections are applied on $m_{\text {eff }}$ and $m_{\mathrm{T} 2}$, while $m_{\mathrm{T}}$ is required to be above $140 \mathrm{GeV}$. VRSS-j23 is equivalent to SRSS-j23, with $m_{\mathrm{T}}$ required to be between $65 \mathrm{GeV}$ and $120 \mathrm{GeV}$ and $m_{\ell j(j)}$ above $130 \mathrm{GeV}$. The total numbers of events observed in data and predicted by the background estimation for the $\ell^{ \pm} \ell^{ \pm}$VRs are shown in Fig. 7, together with the pull estimates.

\section{2. $3 e$ event selection}

Events in the $3 \ell$ signal regions are categorized according to flavor and charge-sign combinations of the leptons in the event. Appropriate selection criteria tailored to each region are applied in order to reject lepton-rich background processes while at the same time maximizing signal significance. The event selections applied in the $3 \ell$ signal regions are summarized in Tables VIII and IX. In differentflavor opposite-sign (DFOS) signal regions, two of the leptons are required to have the same flavor and same-sign (SFSS) electric charge (the SFSS lepton pair), while the third lepton (the DFOS lepton) must have different flavor and opposite charge to the other two leptons. The DFOS lepton and the SFSS lepton closest to it in $\Delta \phi$ (the near lepton) are taken to originate from the Higgs boson decay. The $\Delta R$ between these two leptons is called $\Delta R_{\mathrm{OS} \text {,near }}$, and their invariant mass, which in signal events gives an estimate of the Higgs boson visible mass, is called $m_{\ell_{\mathrm{DFOS}}+\ell_{\text {near }}}$. The azimuthal angle between the two SFSS leptons is called $\Delta \phi_{\mathrm{SS}}$. In same-flavor opposite-sign (SFOS) signal regions, there must be at least one pair of leptons of the same flavor and with opposite-sign charge (the SFOS lepton pair). When only one SFOS lepton pair exists, the invariant mass $m_{\mathrm{SFOS}}^{\min }$ must be greater than $20 \mathrm{GeV}$ and lie outside the $81.2-101.2 \mathrm{GeV}$ interval, to suppress low-mass resonances and Z-rich backgrounds. If two SFOS pairs exist, the chosen SFOS pair has a lower $m_{\mathrm{T}}^{\ell, \text { min }}$ for the third highest $p_{\mathrm{T}}$ lepton, and the invariant mass requirement is applied to this pair. The variable $m_{\mathrm{T}}^{\ell, \min }$, defined in analogy with $m_{\mathrm{T}}^{b \text {,min }}$, is also used to identify the unique transverse mass value obtained from the lepton not in the SFOS pair in events for which only one such pair exists. Both the DFOS and SFOS events are further separated into orthogonal signal regions, depending on whether at least one light jet of $p_{\mathrm{T}}>20 \mathrm{GeV}$ is present in the event or not. Region-dependent requirements are placed on $E_{\mathrm{T}}^{\text {miss }}$, as well as on other kinematic variables.

The reducible FNP lepton background in the $3 \ell$ channel is dominated by $t \bar{t}$ and $Z+$ jets processes, and it is estimated using the same approach as for the $\ell^{ \pm} \ell^{ \pm}$analysis. The irreducible background is dominated by $W Z$ production and is estimated using a dedicated control region. The normalization of the $W Z$ background is constrained in this region to reduce systematic uncertainties due to the $\mathrm{MC}$ modeling and experimental sources. The $W Z$ CR uses a three-lepton selection in which a SFOS pair has an invariant mass in the $Z$ peak region, $81.2<m_{\ell \ell}<101.2 \mathrm{GeV}$, the $E_{\mathrm{T}}^{\text {miss }}$ is above $80 \mathrm{GeV}$, and a $b$-tagging veto is applied. The estimate from the background-only fit leads to a normalization factor of $1.11 \pm 0.13$ for the $W Z$ background and the $E_{\mathrm{T}}^{\text {miss }}$ distribution in the CR is shown in Fig. 6(b). Its validity is cross-checked by comparing the SM estimates with data from a VR (referred to as VR3L-offZ-highMET) where events are required to have $E_{\mathrm{T}}^{\mathrm{miss}}$ above $120 \mathrm{GeV}$ and $m_{\mathrm{SFOS}}^{\min }$ outside of the $Z$ peak region.

The total number of events observed in data and predicted by the background estimation for the $3 \ell \mathrm{VR}$ are shown in Fig. 7, together with the pull estimates. 


\section{SYSTEMATIC UNCERTAINTIES}

Several sources of experimental and theoretical systematic uncertainties in the signal and background estimates are considered in these analyses. Their impact is reduced through the normalization of the dominant backgrounds in the control regions defined with kinematic selections resembling those of the corresponding signal region. Experimental and theoretical uncertainties are included as nuisance parameters with Gaussian constraints in the likelihood fits, taking into account correlations between different regions. Uncertainties due to the numbers of events in the CRs are also included in the fit for each region.

Theory uncertainties for $t \bar{t}$ processes are dominant for the $0 \ell b \bar{b}$ and $1 \ell b \bar{b}$ analysis channels, ranging from $15 \%$ to $20 \%$ for the $1 \ell b \bar{b}$ channel to nearly $50 \%$ for the low-mass signal region (SRHad-Low) of the $0 \ell b \bar{b}$ analysis. Generator uncertainties are assessed by comparing Powheg+Pythia6 with SHERPa 2.2.1, and the parton shower models are tested by comparing PowHEG+ Pythia6 with Powheg + Herwig ++ . Scale variations are evaluated by varying the $h_{\text {damp }}$ parameter between $m_{\text {top }}$ and $2 \times m_{\text {top }}$, and the renormalization and factorization scales up and down by a factor of two. Systematic uncertainties in the contributions from single-top production also account for the impact of interference terms between single-resonant and doubleresonant top-quark production. Statistical uncertainties are included via the control regions in the data by which the processes are normalized and the size of the simulation samples used for evaluating theoretical systematic uncertainties. Relaxed selections are used to reduce the statistical uncertainty of theory estimates of top-quark contributions. In particular, the $m_{\mathrm{CT}}$ selection is loosened for both $0 \ell b \bar{b}$ and $1 \ell b \bar{b}$, as are the $m_{\mathrm{T}}^{b \text {,min }}$ and $m_{\text {eff }}$ selections for the $0 \ell b \bar{b}$ channel. The $Z+$ jets and $W+$ jets modeling uncertainties are estimated using the nominal SHERPA 2.2.1 samples by considering different merging (CKKW-L) and resummation scales, PDF variations from the NNPDF30NNLO replicas, as well as the envelope of changes resulting from sevenpoint scale variations of the renormalization and factorization scales. The various components are added in quadrature.

Theory uncertainties in both the $W h$ production cross section and the modeling of the $W h$ final state also contribute to the uncertainty of the peaking backgrounds in the $1 \ell \gamma \gamma$ analysis. They are estimated by varying the nominal PDF error sets, the QCD factorization scale, the parameters associated with the underlying event and parton shower, and the NLO electroweak correction factors associated with the simulation of the $W h$ process. These variations lead to a fractional uncertainty of $\pm 5.5 \%$ in the expected contribution of $W h$ production to the $1 \ell \gamma \gamma$ SRs.
Theory uncertainties related to the estimation of the $W Z$ background are among the most significant for the multilepton analysis channels $\left(\ell^{ \pm} \ell^{ \pm}\right.$and $\left.3 \ell\right)$. The effects of PDF choice and the scale of the strong coupling constant, $\alpha_{\mathrm{S}}$, on the $W Z$ background are assessed using the same procedure as described above for scale variations in top-quark production processes: by varying the relevant parameters and measuring the impact on the quantities of interest.

The dominant detector-related systematic effects differ depending on the analysis channel. Experimental uncertainties related to the jet energy resolution are significant in the case of $1 \ell b \bar{b}$, accounting for nearly $20 \%$ of the total systematic uncertainty on the background estimation in the SR1Lbb-Medium region. Uncertainties related to the jet energy scale contribute to approximately a $30 \%$ systematic uncertainty in the SRHad-High region. Uncertainties of the $b$-tagging efficiency and mistagging rates are subdominant for $1 \ell b \bar{b}$ and $0 \ell b \bar{b}$ channels, and are estimated by varying the $\eta-, p_{\mathrm{T}^{-}}$and flavor-dependent scale factors applied to each jet in the simulation within a range that reflects the systematic uncertainty of the measured tagging efficiency and mistagging rates. The effects of experimental uncertainty in the $1 \ell \gamma \gamma$ channel are dominated by uncertainties in the photon, lepton and jet energy scale and resolution. The uncertainty on the contribution from nonpeaking background is dominated by the effect of the limited number of events in the $m_{\gamma \gamma}$ sidebands. An additional contribution from the uncertainty in the shape of the nonpeaking background $m_{\gamma \gamma}$ distribution was found to be negligible. The $\ell^{ \pm} \ell^{ \pm} / 3 \ell$ channels are dominated in several signal regions by experimental systematic uncertainties related to the estimation of background contributions due to FNP leptons. These systematic uncertainties are evaluated with various studies including $Z \rightarrow \ell \ell$ efficiency comparisons, variations of kinematic selections, modifications to the definition of the control regions, and alternative trigger selections. For the $\ell^{ \pm} \ell^{ \pm}$channel, these are the dominant uncertainties and have similar contributions from each source.

The dominant systematic uncertainties in the various signal regions are summarized in Table $\mathrm{X}$.

\section{RESULTS}

No significant differences between the observed and expected yields are found in the search regions for each of the analysis channels considered. The results are translated into upper limits on contributions from physics processes beyond the SM (BSM) for each signal region and are used to set exclusion limits at the $95 \%$ confidence level (C.L.) on the common mass of the charginos and next-to-lightest neutralinos for various values of the LSP mass in the simplified model considered in the analysis. 
TABLE X. Dominant systematic uncertainties in the background estimates in the various signal regions, expressed in terms of number of events. Individual uncertainties can be correlated, and do not necessarily add up quadratically to the total background uncertainty. For the $3 \ell$ channel, numbers in parentheses indicate the results for the (b) signal region in each case.

\begin{tabular}{lcc}
\hline \hline $0 \ell b \bar{b}$ channel & & \\
\hline Uncertainty of region & SRHad-High & SRHad-Low \\
Total background expectation & 2.5 & 8 \\
Total background uncertainty & \pm 1.3 & \pm 4 \\
Systematic, experimental & \pm 0.9 & \pm 1.2 \\
Systematic, theoretical & \pm 0.7 & \pm 3 \\
Statistical, MC samples & \pm 0.5 & \pm 0.8 \\
Statistical, $\mu_{\mathrm{TT}, \mathrm{ST}, \mathrm{Zj}}$ scale-factors & \pm 0.25 & \pm 0.5 \\
\hline \hline
\end{tabular}

\section{$1 \ell b \bar{b}$ channel}

Uncertainty of region

Total background expectation

Total background uncertainty

Systematic, experimental

Systematic, theoretical

Statistical, MC samples

Statistical, $\mu_{\mathrm{TT}, \mathrm{ST}, \mathrm{Wj}}$ scale factors

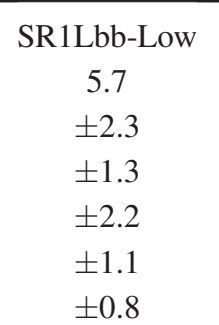

SR1Lbb-Medium

2.8

$\pm 1.0$

$\pm 0.7$

$\pm 0.9$

$\pm 0.5$

$\pm 0.6$
SR1Lbb-High

4.6

$\pm 1.2$

$\pm 0.6$

$\pm 0.7$

$\pm 0.6$

$\pm 1.3$

\section{$1 \ell \gamma \gamma$ channel}

Uncertainty of region

Total background expectation

Total background uncertainty

Systematic, experimental

Systematic, theoretical

Statistical, MC samples

Statistical, nonpeaking

\begin{tabular}{cc} 
SR1L $\gamma \gamma-\mathrm{a}$ & SR1L $\gamma \gamma-\mathrm{b}$ \\
0.36 & 5.3 \\
\pm 0.22 & \pm 1.0 \\
\pm 0.018 & \pm 0.27 \\
\pm 0.008 & \pm 0.11 \\
\pm 0.006 & \pm 0.024 \\
\pm 0.22 & \pm 0.9 \\
\hline
\end{tabular}

$\ell^{ \pm} \ell^{ \pm}$channel

Uncertainty of region

Total background expectation

Total background uncertainty

Systematic, experimental

Systematic, theoretical

Statistical, MC samples

$\begin{array}{cc}\text { SRSS-j1 } & \text { SRSS-j23 } \\ 6.7 & 5.3 \\ \pm 2.2 & \pm 1.6 \\ \pm 2.1 & \pm 1.6 . \\ \pm 0.21 & \pm 0.28 \\ \pm 0.4 & \pm 0.34\end{array}$

SRSS-j1

$\pm 2.2$

\begin{tabular}{lcccc}
\hline \hline $3 \ell$ channel & \multicolumn{5}{l}{} \\
\hline Uncertainty of region & SR3L-DFOS-0J & SR3L-DFOS-1Ja(b) & SR3L-SFOS-0Ja(b) & SR3L-SFOS-1J \\
Total background expectation & 2.05 & $8(1.7)$ & $3.8(2.37)$ & 11.4 \\
Total background uncertainty & \pm 0.98 & $\pm 4( \pm 0.7)$ & $\pm 1.7( \pm 0.96)$ & \pm 2.6 \\
Systematic, experimental & \pm 0.8 & $\pm 4( \pm 0.5)$ & $\pm 1.7( \pm 0.8)$ & \pm 2.0 \\
Systematic, theoretical & \pm 0.11 & $\pm 0.25( \pm 0.16)$ & $\pm 0.15( \pm 0.22)$ & \pm 1.5 \\
Statistical, MC samples & \pm 0.6 & $\pm 1.2( \pm 0.4)$ & $\pm 0.6( \pm 0.4)$ & \pm 0.9 \\
Statistical, $\mu_{\mathrm{WZ}}$ scale factors & \pm 0.022 & $\pm 0.12( \pm 0.06)$ & $\pm 0.30( \pm 0.24)$ & \pm 0.9 \\
\hline \hline
\end{tabular}


TABLE XI. Event yields and SM expectation for the $0 \ell b \bar{b}$ channel after the background-only fit for the SRHad-High and SRHad-Low regions. The errors shown are the statistical plus systematic uncertainties. Uncertainties in the fitted yields are symmetric by construction, where the negative error is truncated when reaching zero event yield.

\begin{tabular}{lcc}
\hline \hline SR channels & SRHad-High & SRHad-Low \\
\hline Observed events & 1 & 7 \\
Fitted background events & $2.5 \pm 1.3$ & $8 \pm 4$ \\
$t \bar{t}$ & $1.1 \pm 0.9$ & $4 \pm 4$ \\
Single top $(W t)$ & $0.15_{-0.15}^{+0.16}$ & $0.44 \pm 0.33$ \\
$W+$ jets & $0.1_{-0.1}^{+0.3}$ & $1.0 \pm 0.7$ \\
$Z+$ jets & $1.0 \pm 0.7$ & $1.7 \pm 1.0$ \\
$t \bar{t}+V$ & $0.09 \pm 0.03$ & $0.40 \pm 0.08$ \\
Diboson & $<0.01$ & $0.3_{-0.3}^{+0.4}$ \\
\hline \hline
\end{tabular}

Table XI provides the event yields and SM expectation for the $0 \ell b \bar{b}$ analysis channel in the two signal regions (SRHad-High, SRHad-Low) after the background-only fit. The errors shown are the statistical plus systematic uncertainties. Table XII reports the observed number of events in the three SRs for the $1 \ell b \bar{b}$ signature compared to the SM expectations. Good agreement is found between data and SM predictions for both $0 \ell b \bar{b}$ signal regions and two of the three $1 \ell b \bar{b}$ signal regions; SR1Lbb-Medium exhibits a mild excess. For the $1 \ell \gamma \gamma$ channel, the expected SM backgrounds, broken down by source, are summarized along with their estimated uncertainties in Table XIII. A mild excess of observed events relative to expected SM backgrounds is seen in each of the two signal regions, corresponding to $p_{0}$-values of 0.027 and 0.087

TABLE XII. Event yields and SM expectation after the background-only fit in the $1 \ell b \bar{b}$ channel for the SR1Lbb-Low, SR1Lbb-Medium, and SR1Lbb-High regions. The category "Others" includes contributions from three- and four-top production and SM Higgs processes. The errors shown are the statistical plus systematic uncertainties. Uncertainties in the fitted yields are symmetric by construction, where the negative error is truncated when reaching zero event yield.

\begin{tabular}{lccc}
\hline \hline SR channels & $\begin{array}{c}\text { SR1Lbb- } \\
\text { Low }\end{array}$ & $\begin{array}{c}\text { SR1Lbb- } \\
\text { Medium }\end{array}$ & $\begin{array}{c}\text { SR1Lbb- } \\
\text { High }\end{array}$ \\
\hline Observed events & 6 & 7 & 5 \\
Fitted background & $5.7 \pm 2.3$ & $2.8 \pm 1.0$ & $4.6 \pm 1.2$ \\
$\quad$ events & $3.4 \pm 2.9$ & $1.4 \pm 1.0$ & $1.1 \pm 0.6$ \\
$t \bar{t}$ & $1.4_{-1.4}^{+1.4}$ & $0.8_{-0.8}^{+0.9}$ & $1.2 \pm 1.1$ \\
Single top $(W t)$ & $0.6 \pm 0.4$ & $0.20 \pm 0.11$ & $1.6 \pm 0.6$ \\
$W+$ jets & $0.10 \pm 0.04$ & $0.32 \pm 0.09$ & $0.54 \pm 0.14$ \\
$t \bar{t}+V$ & $0.12_{-0.12}^{+0.15}$ & $0.05 \pm 0.03$ & $0.08 \pm 0.02$ \\
Diboson & $0.10 \pm 0.05$ & $0.03 \pm 0.01$ & $0.04 \pm 0.02$ \\
Others &
\end{tabular}

TABLE XIII. Expected numbers of peaking and nonpeaking SM background events in the $1 \ell \gamma \gamma$ channel for SR1L $\gamma \gamma$-a and SR1L $\gamma \gamma$-b. Nonpeaking-background uncertainty is dominated by the statistical uncertainty in the sideband fits. The peaking background uncertainties include both theoretical (production rate) and experimental (detector effect) contributions, as described in the text. The uncertainties in the Wh and Other peaking backgrounds are taken to be fully correlated. Also shown are the observed numbers of events in SR1L $\gamma \gamma$-a and SR1L $\gamma \gamma$-b.

\begin{tabular}{lcc}
\hline \hline SR channels & SR1L $\gamma \gamma-\mathrm{a}$ & SR1L $\gamma \gamma-\mathrm{b}$ \\
\hline Observed events & 2 & 9 \\
Total background events & $0.37 \pm 0.22$ & $5.3 \pm 1.0$ \\
Wh background & $0.09 \pm 0.01$ & $1.8 \pm 0.3$ \\
Other peaking backgrounds & $0.04 \pm 0.01$ & $0.19 \pm 0.02$ \\
Nonpeaking background & $0.22 \pm 0.22$ & $3.3 \pm 0.9$ \\
\hline \hline
\end{tabular}

TABLE XIV. Event yields and SM expectation for the $\ell^{ \pm} \ell^{ \pm}$ signal regions SRSS-j1 and SRSS-j23 after the background-only fit. The category "Rare" includes contributions from triboson, three- and four-top production and SM Higgs processes. The errors shown are the statistical plus systematic uncertainties.

\begin{tabular}{lcc}
\hline \hline SR channels & SRSS-j1 & SRSS-j23 \\
\hline Observed events & 2 & 8 \\
Fitted background events & $6.7 \pm 2.2$ & $5.3 \pm 1.6$ \\
FNP events & $3.3 \pm 2.1$ & $1.8 \pm 1.5$ \\
$W Z$ & $2.2 \pm 0.5$ & $1.9 \pm 0.6$ \\
Rare & $0.44 \pm 0.13$ & $0.73 \pm 0.17$ \\
$t \bar{t}+V$ & $0.12 \pm 0.05$ & $0.14 \pm 0.05$ \\
$W W$ & $0.17 \pm 0.03$ & $0.51 \pm 0.07$ \\
$Z Z$ & $0.06 \pm 0.03$ & $0.07 \pm 0.04$ \\
Charge-flip events & $0.47 \pm 0.07$ & $0.27 \pm 0.03$ \\
\hline \hline
\end{tabular}

TABLE XV. Event yields and SM expectation after the background-only fit in the $3 e$ channel for the SR3L-SFOS0Ja, SR3L-SFOS-0Jb and SR3L-SFOS-1J regions. The category "Higgs" includes contributions from $t \bar{t}+$ Higgs boson production. The errors shown are the statistical plus systematic uncertainties. Uncertainties in the fitted yields are symmetric by construction, where the negative error is truncated when reaching zero event yield.

\begin{tabular}{lccc}
\hline \hline SR channels & $\begin{array}{c}\text { SR3L- } \\
\text { SFOS-0Ja }\end{array}$ & $\begin{array}{c}\text { SR3L- } \\
\text { SFOS-0Jb }\end{array}$ & $\begin{array}{c}\text { SR3L- } \\
\text { SFOS-1J }\end{array}$ \\
\hline Observed events & 0 & 3 & 11 \\
Fitted background & $3.8 \pm 1.7$ & $2.4 \pm 1.0$ & $11.5 \pm 2.6$ \\
$\quad$ events & $2.5 \pm 1.2$ & $2.0 \pm 0.9$ & $7.4 \pm 2.3$ \\
$W Z$ & $0.10 \pm 0.04$ & $0.07 \pm 0.02$ & $0.29 \pm 0.09$ \\
$Z Z$ & $0.09 \pm 0.03$ & $0.02 \pm 0.01$ & $1.9 \pm 0.5$ \\
$t \bar{t}+V$ & $0.57 \pm 0.29$ & $0.16 \pm 0.08$ & $1.4 \pm 0.4$ \\
Tribosons & $0.24_{-0.24}^{+0.25}$ & $0.07 \pm 0.07$ & $0.07 \pm 0.04$ \\
Higgs SM & $0.27_{-0.27}^{+0.31}$ & $0.11_{-0.11}^{+0.20}$ & $0.4_{-0.4}^{+0.5}$ \\
FNP events & & & \\
\hline \hline
\end{tabular}


TABLE XVI. Event yields and SM expectation after the background-only fit in the $3 e$ channel for the SR3L-DFOS-0J, SR3L-DFOS-1Ja and SR3L-DFOS-1Jb regions. The category "Higgs" includes contributions from $t \bar{t}+$ Higgs boson production. The errors shown are the statistical plus systematic uncertainties. Uncertainties in the fitted yields are symmetric by construction, where the negative error is truncated when reaching zero event yield.

\begin{tabular}{lccc}
\hline \hline SR channels & $\begin{array}{c}\text { SR3L- } \\
\text { DFOS-0J }\end{array}$ & $\begin{array}{c}\text { SR3L- } \\
\text { DFOS-1Ja }\end{array}$ & $\begin{array}{c}\text { SR3L- } \\
\text { DFOS-1Jb }\end{array}$ \\
\hline $\begin{array}{l}\text { Observed events } \\
\text { Fitted background }\end{array}$ & 0 & 7 & 1 \\
$\quad$ events & $2.1 \pm 1.0$ & $8.3 \pm 3.8$ & $1.7 \pm 0.7$ \\
$W Z$ & $0.18 \pm 0.13$ & $1.01 \pm 0.27$ & $0.54 \pm 0.16$ \\
$Z Z$ & $0.0017 \pm 0.0012$ & $0.06 \pm 0.02$ & $0.03 \pm 0.01$ \\
$t \bar{t}+V$ & $0.0013 \pm 0.0013$ & $0.79 \pm 0.29$ & $0.43 \pm 0.16$ \\
Tribosons & $0.52 \pm 0.28$ & $0.66 \pm 0.22$ & $0.23 \pm 0.08$ \\
Higgs SM & $0.39 \pm 0.15$ & $0.1_{-0.1}^{+0.5}$ & $0.05 \pm 0.04$ \\
FNP & $1.0 \pm 0.9$ & $5.6 \pm 3.8$ & $0.4_{-0.4}^{+0.6}$ \\
\hline \hline
\end{tabular}

for SR1L $\gamma \gamma-\mathrm{a}$ and SR1L $\gamma \gamma-\mathrm{b}$, respectively. Finally, Tables XIV-XVI report the observed and predicted SM backgrounds for the various multilepton signal regions.

Table XVII summarizes the observed $\left(S_{\mathrm{obs}}^{95}\right)$ and expected $\left(S_{\exp }^{95}\right)$ 95\% C.L. upper limits on the number of signal events and on the observed visible cross section, $\sigma_{\mathrm{vis}}$, for all channels and signal regions. Upper limits on contributions from new physics processes are estimated using the socalled model-independent fit. The $\mathrm{CL}_{\mathrm{s}}$ method $[78,79]$ is used to derive the confidence level of the exclusion for a particular signal model; signal models with a $\mathrm{CL}_{\mathrm{s}}$ value below 0.05 are excluded at $95 \%$ C.L. When normalized to the integrated luminosity of the data sample, results can be interpreted as corresponding to observed upper limits on $\sigma_{\text {vis }}$, defined as the product of the production cross section,

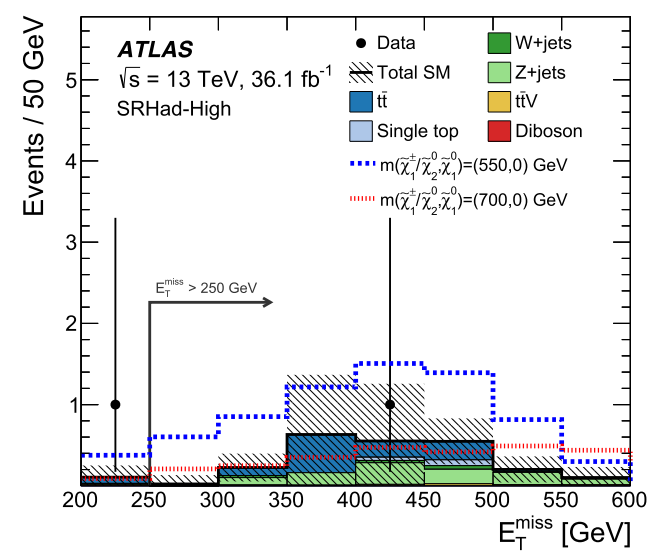

(a) $E_{\mathrm{T}}^{\text {miss }}$ in SRHad-High
TABLE XVII. From left to right, the observed 95\% C.L. upper limits on the visible cross sections $\sigma_{\text {vis }}$, the observed $\left(S_{\text {obs }}^{95}\right)$ and expected $\left(S_{\exp }^{95}\right) 95 \%$ C.L. upper limits on the number of signal events with $\pm 1 \sigma$ excursions of the expectation, and the discovery $p$-value $\left(p_{0}\right)$, truncated at 0.5 .

\begin{tabular}{lcccc}
\hline \hline & $\sigma_{\text {vis }}[\mathrm{fb}]$ & $S_{\text {obs }}^{95}$ & $S_{\text {exp }}^{95}$ & $p_{0}$-value \\
\hline SRHad-Low & 0.26 & 9.4 & $9.5_{-1.9}^{+3.3}$ & 0.50 \\
SRHad-High & 0.10 & 3.6 & $4.3_{-1.0}^{+1.6}$ & 0.50 \\
SR1Lbb-Low & 0.23 & 8.3 & $8.0_{-2.2}^{+3.3}$ & 0.46 \\
SR1Lbb-Medium & 0.28 & 10.0 & $5.6_{-1.7}^{+2.9}$ & 0.04 \\
SR1Lbb-High & 0.18 & 6.4 & $6.1_{-1.9}^{+3.1}$ & 0.44 \\
SR1L $\gamma \gamma$-a & 0.15 & 5.5 & $3.2_{-0.1}^{+0.9}$ & 0.03 \\
SR1L $\gamma \gamma$-b & 0.28 & 10.1 & $6.4_{-1.6}^{+2.6}$ & 0.09 \\
SRSS-j1 & 0.12 & 4.2 & $6.1_{-1.5}^{+2.7}$ & 0.50 \\
SRSS-j23 & 0.27 & 9.9 & $6.6_{-1.1}^{+3.4}$ & 0.17 \\
SR3L-SFOS-0Ja & 0.08 & 3.0 & $4.4_{-1.3}^{+1.9}$ & 0.47 \\
SR3L-SFOS-0Jb & 0.16 & 5.9 & $5.0_{-1.2}^{+2.0}$ & 0.35 \\
SR3L-SFOS-1J & 0.26 & 9.2 & $9.4_{-2.5}^{+3.8}$ & 0.50 \\
SR3L-DFOS-0J & 0.08 & 3.0 & $3.8_{-0.9}^{+1.4}$ & 0.43 \\
SR3L-DFOS-1Ja & 0.25 & 9.0 & $9.2_{-2.3}^{+3.3}$ & 0.50 \\
SR3L-DFOS-1Jb & 0.10 & 3.7 & $4.0_{-0.5}^{+1.6}$ & 0.50 \\
\hline \hline
\end{tabular}

the acceptance and the selection efficiency of a BSM signal. The $p_{0}$-values, which represent the probability of the SM background alone to fluctuate to the observed number of events or higher, are also provided.

For the $0 \ell b \bar{b}$ analysis channel, Fig. 8 shows the distributions of $E_{\mathrm{T}}^{\mathrm{miss}}$ and $m_{b \bar{b}}$ in the SRHad-High and SRHad-Low SRs, respectively. Fig. 9 shows the data distributions of $m_{\mathrm{CT}}$ and $E_{\mathrm{T}}^{\text {miss }}$ for the $1 \ell b \bar{b}$ analysis in the SR1Lbb-High and SR1Lbb-Medium SRs compared to the SM expectations. Fig. 10 shows the $m_{\gamma \gamma}$ distribution, separately for SR1L $\gamma \gamma$-a and SR1L $\gamma \gamma$-b, before the final

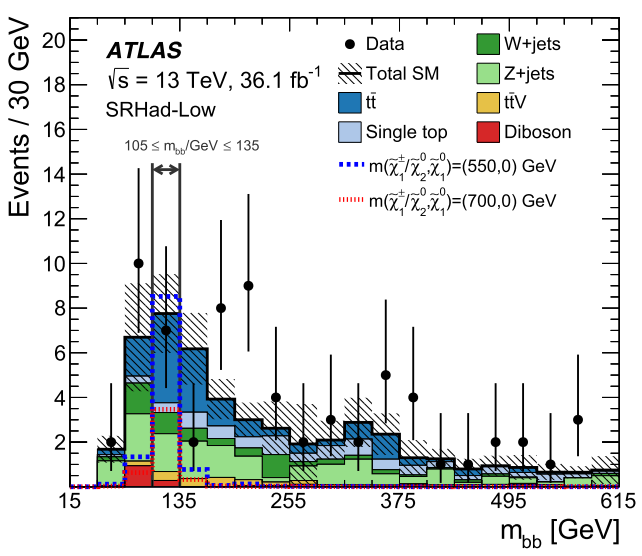

(b) $m_{b \bar{b}}$ in SRHad-Low

FIG. 8. Data and SM predictions in SRs for the $0 \ell b \bar{b}$ analysis for (a) $E_{\mathrm{T}}^{\text {miss }}$ in SRHad-High and (b) $m_{b \bar{b}}$ in SRHad-Low. All SRs selections but the one on the quantity shown are applied. All uncertainties are included in the uncertainty band. Two example SUSY models are superimposed for illustrative purposes. 


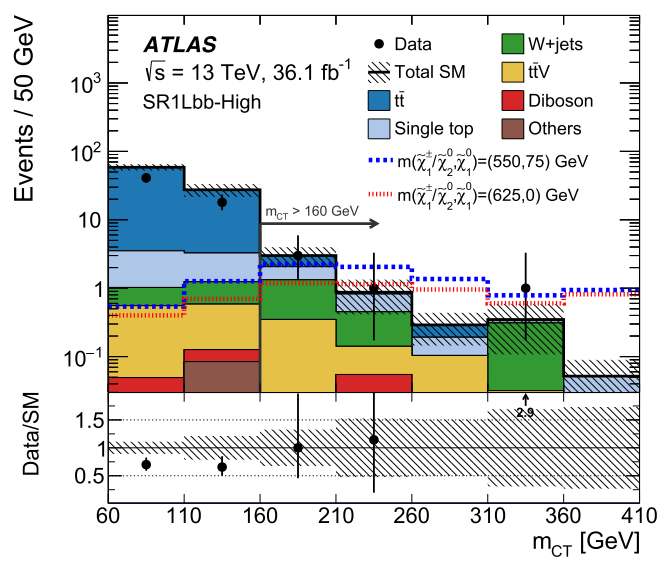

(a) $m_{\mathrm{CT}}$ in SR1Lbb-High

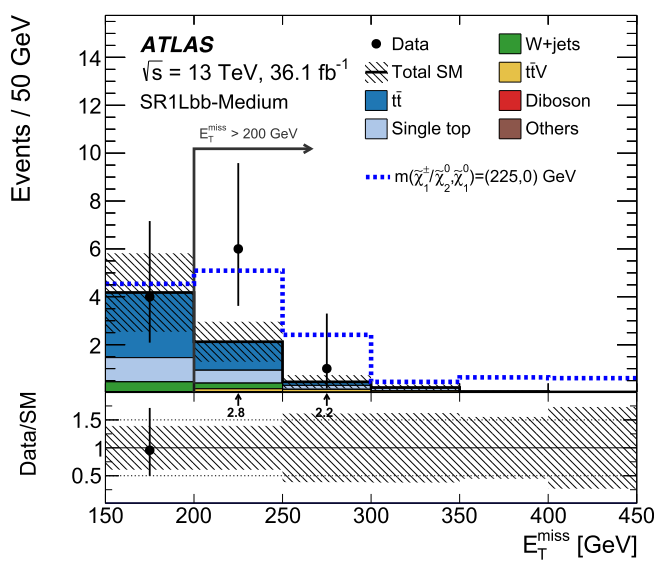

(b) $E_{\mathrm{T}}^{\mathrm{miss}}$ in SR1Lbb-Medium

FIG. 9. Data and SM predictions in SRs for the $1 \ell b \bar{b}$ analysis for (a) $m_{\mathrm{CT}}$ in SR1Lbb-High and (b) $E_{\mathrm{T}}^{\mathrm{miss}}$ in SR1Lbb-Medium. All SRs selections but the one on the quantity shown are applied. All uncertainties are included in the uncertainty band. Example SUSY models are superimposed for illustrative purposes.

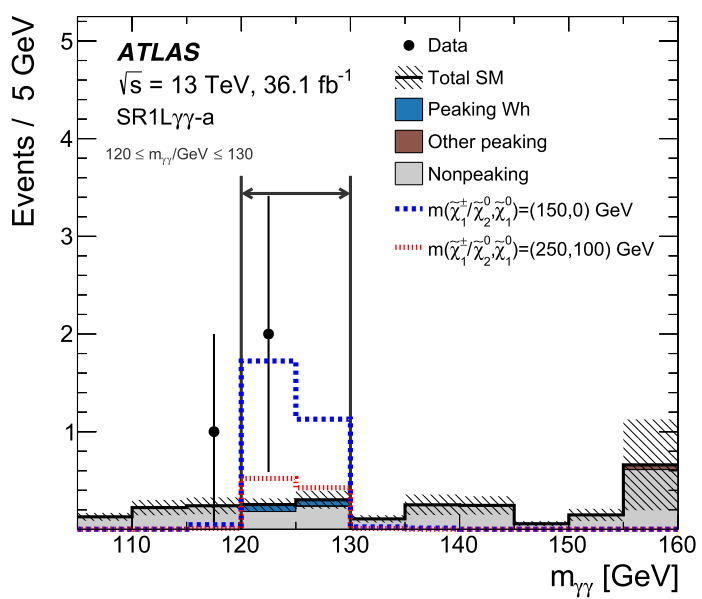

(a) $m_{\gamma \gamma}$ in SR1L $\gamma \gamma$-a

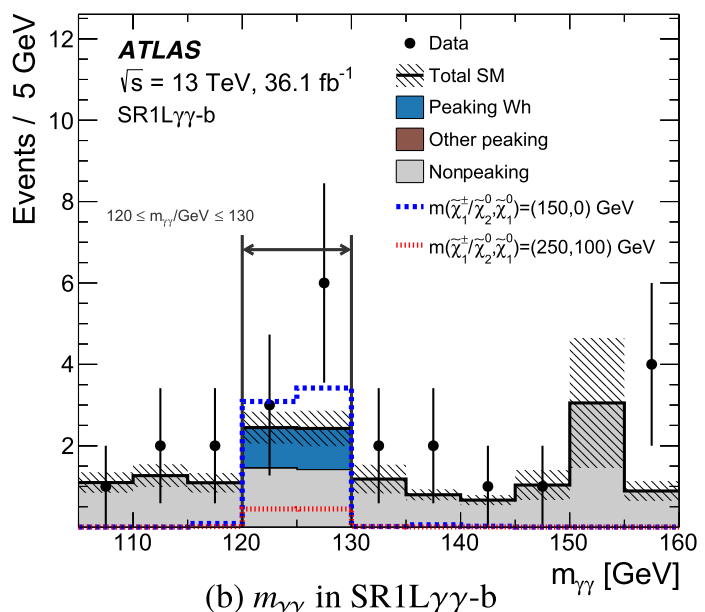

FIG. 10. Distributions of $m_{\gamma \gamma}$ before the final requirement on $m_{\gamma \gamma}$ in (a) SR1L $\gamma \gamma$-a and (b) SR1L $\gamma \gamma$-b. The expected contributions from both the peaking and nonpeaking backgrounds are shown as stacked colored histograms. Two example SUSY models are superimposed for illustrative purposes. selection applied to $m_{\gamma \gamma}$. Observed and predicted distributions of $m_{\ell j(j)}$ (SRSS-j1) and $m_{\mathrm{T} 2}$ (SRSS-j23) for the $\ell^{ \pm} \ell^{ \pm}$ signature are shown in Fig. 11. The data agree well with the SM expectations in all distributions and for all channels, and no significant deviations are observed.

Figure 12(a) shows the observed and expected exclusion contours for the simplified models shown in Fig. 1(a) for the $0 \ell b \bar{b}$ analysis channel. The signal region (either SRHad-High or SRHad-Low) used for each hypothesis for the $\tilde{\chi}_{1}^{ \pm} / \tilde{\chi}_{2}^{0}-\tilde{\chi}_{1}^{0}$ mass difference is chosen according to which has better expected sensitivity. Experimental and theoretical systematic uncertainties, as described in Sec. VII, are applied to background and signal samples. Figure 12(b) shows the observed and expected exclusion contours obtained for the $1 \ell b \bar{b}$ channel: in this case, a statistical combination of the results from the three signal regions is performed. Due to the large branching ratio of the Higgs boson into $b$-quark pairs, the sensitivity of the $0 \ell b \bar{b}$ and $1 \ell b \bar{b}$ channels is best at high masses of the chargino and next-to-lightest neutralinos, and exclusion limits up to $680 \mathrm{GeV}$ are achieved for massless neutralinos.

Figure 12(c) shows the expected limits obtained for the $1 \ell \gamma \gamma$ channel. The excess of events observed in this signal region precludes an exclusion limit, even when combining the two SRs. Exclusion limits for the $\ell^{ \pm} \ell^{ \pm}$analysis, obtained with a statistical combination of the two signal regions, are shown in Fig. 12(d). This channel is primarily sensitive at low $\tilde{\chi}_{1}^{ \pm} / \tilde{\chi}_{2}^{0}$ mass values and slightly extends the observed exclusion for models with small mass difference between $\tilde{\chi}_{1}^{ \pm} / \tilde{\chi}_{2}^{0}$ and $\tilde{\chi}_{1}^{0}$. Finally, the sensitivity of the $3 \ell$ channel is small compared to other analysis channels due in large part to not considering hadronic $\tau$ decay modes. The observed and expected cross-section exclusion contours, based on the statistical combination of the $3 \ell$ SRs, are 


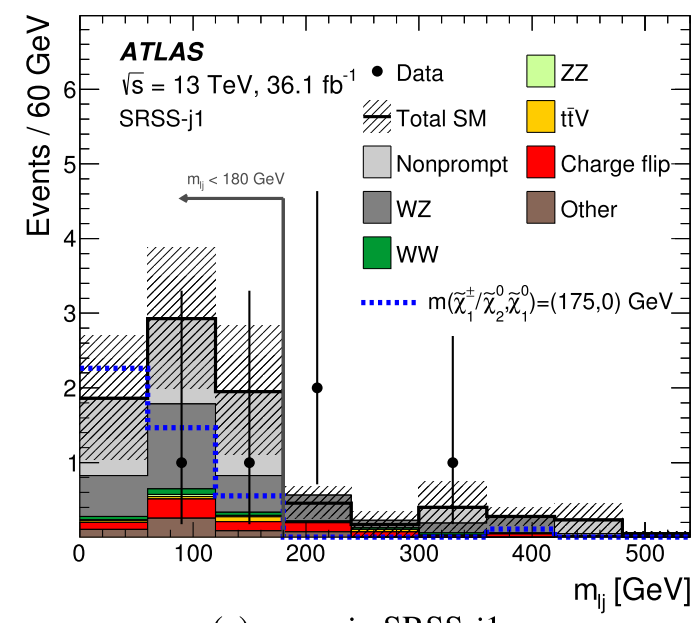

(a) $m_{\ell j(j)}$ in SRSS-j1

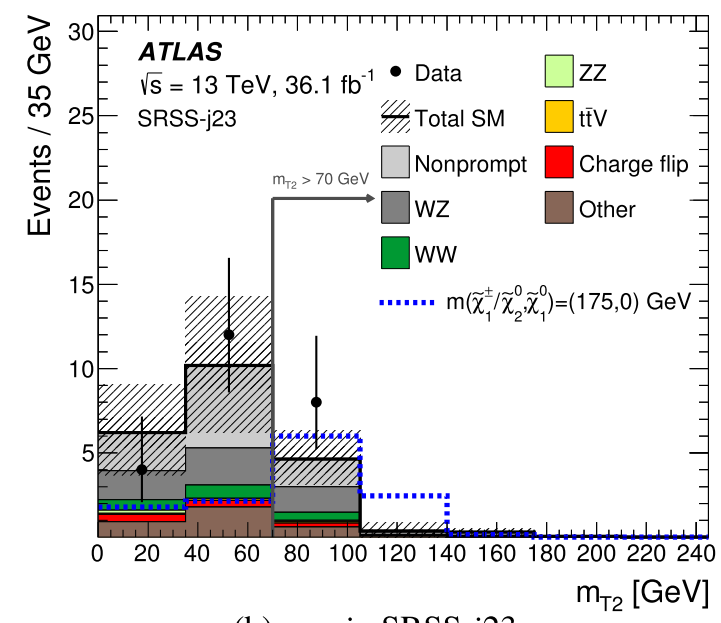

(b) $m_{\mathrm{T} 2}$ in SRSS-j23

FIG. 11. Observed and predicted distributions for (a) $m_{\ell j(j)}$ in SRSS-j1 and (b) $m_{\mathrm{T} 2}$ in SRSS-j23. All SRs selections but the one on the quantity shown are applied. All uncertainties are included in the uncertainty band. An example SUSY model is superimposed for illustrative purposes.

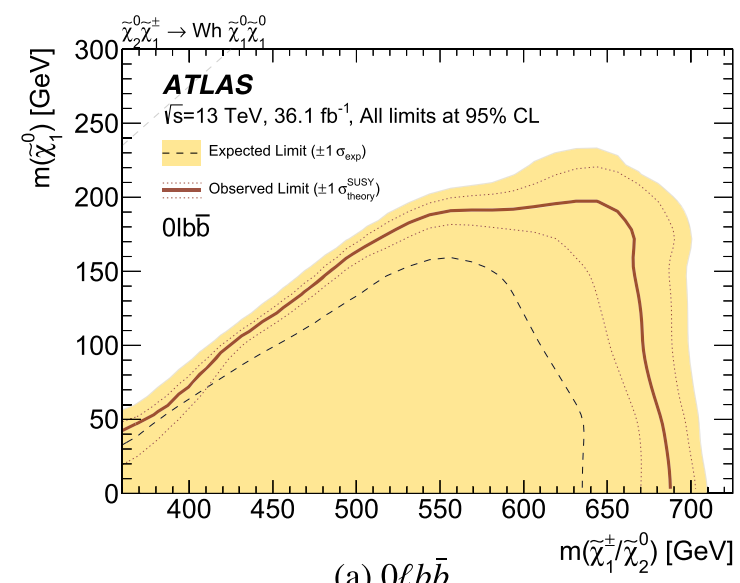

(a) $0 \ell b \bar{b}$

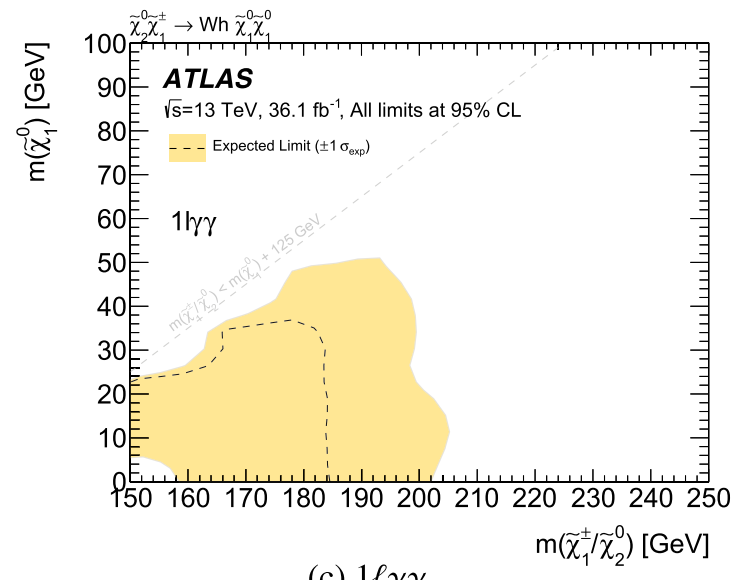

(c) $1 \ell \gamma \gamma$

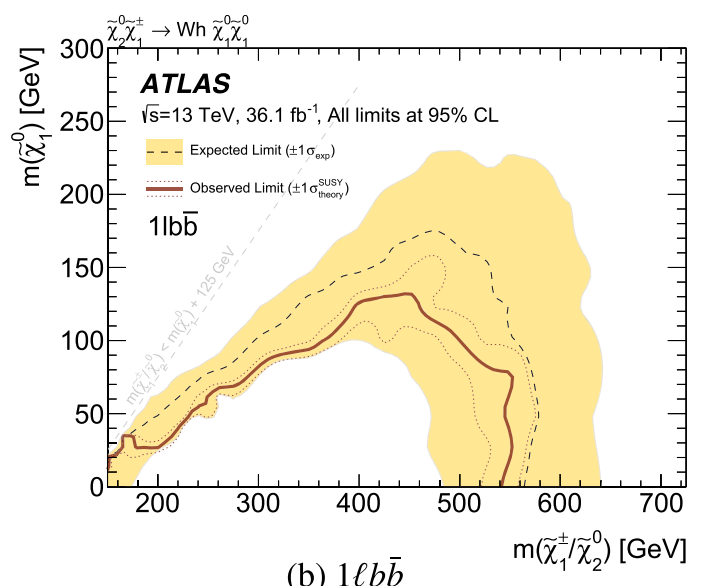

(b) $1 \ell b \bar{b}$

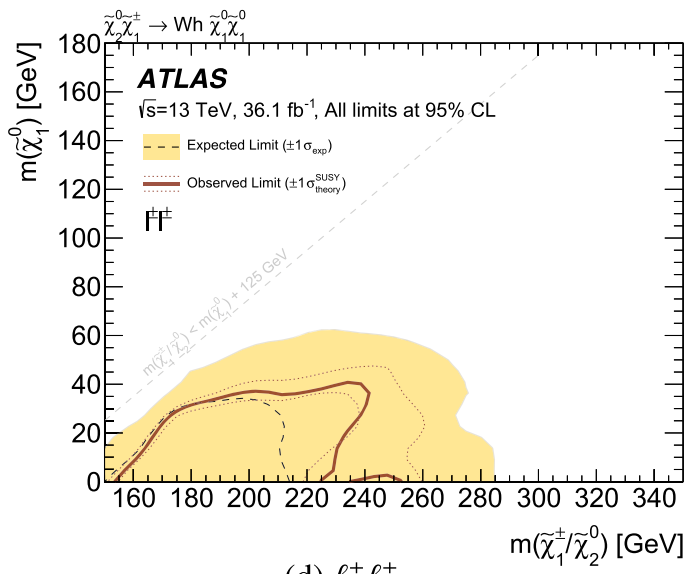

(d) $\ell^{ \pm} \ell^{ \pm}$

FIG. 12. The expected and observed exclusion for the $0 \ell b \bar{b}, 1 \ell b \bar{b}, 1 \ell \gamma \gamma$, and $\ell^{ \pm} \ell^{ \pm}$channels. Experimental and theoretical systematic uncertainties, as described in Sec. VII, are applied to background and signal samples and illustrated by the yellow band and the red dotted contour lines, respectively. The red dotted lines indicate the \pm 1 standard-deviation variation on the observed exclusion limit due to theoretical uncertainties in the signal cross section. 


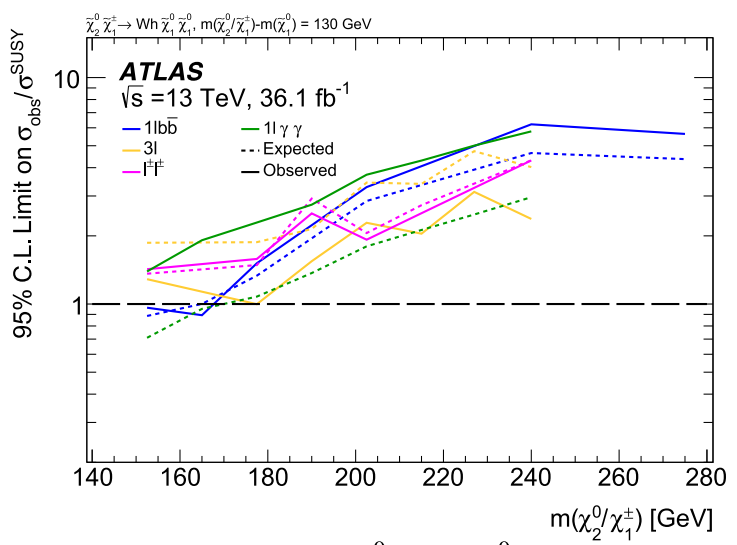

(a) 1-D exclusion for $m\left(\tilde{\chi}_{2}^{0}\right)-m\left(\tilde{\chi}_{1}^{0}\right)=130 \mathrm{GeV}$.

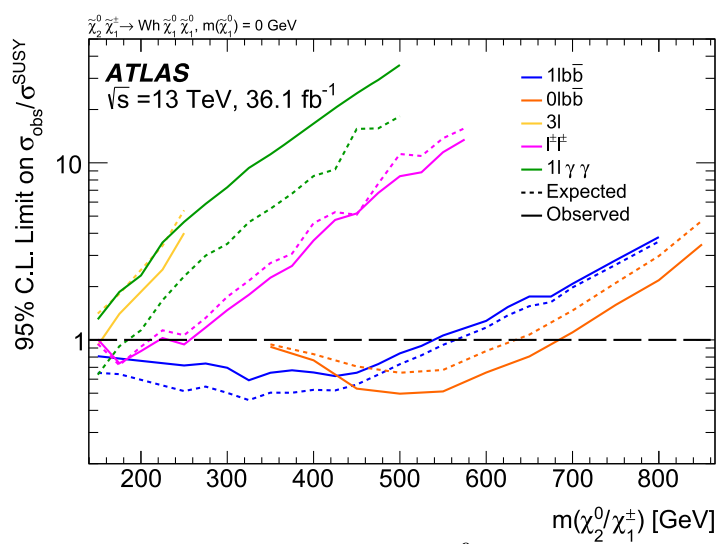

(b) 1-D exclusion for $m\left(\tilde{\chi}_{1}^{0}\right)=0 \mathrm{GeV}$.

FIG. 13. The expected and observed cross-section exclusion as a function of the $\tilde{\chi}_{2}^{0} / \tilde{\chi}_{1}^{0}$ masses for all channels (a) for signal models with $m\left(\tilde{\chi}_{2}^{0}\right)-m\left(\tilde{\chi}_{1}^{0}\right)=130 \mathrm{GeV}$ and (b) assuming $m\left(\tilde{\chi}_{1}^{0}\right)=0 \mathrm{GeV}$.

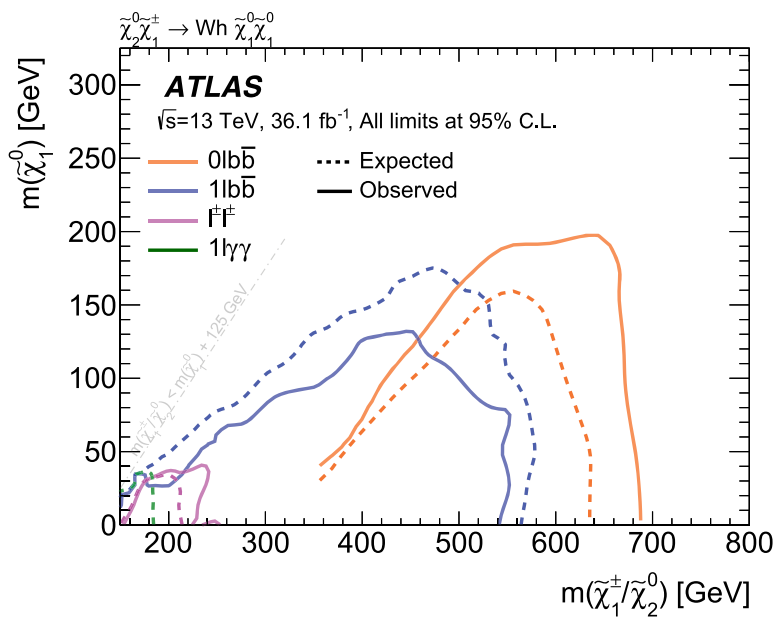

FIG. 14. Comparison of the expected and observed exclusions for each analysis channel studied. Only the expected exclusion is shown for the $1 \ell \gamma \gamma$ channel since the observed exclusion does not appear due to the excess observed. compared with those of other channels in Fig. 13(a) and Fig. 13(b) as a function of the $\tilde{\chi}_{1}^{ \pm} / \tilde{\chi}_{2}^{0}$ masses for $m\left(\tilde{\chi}_{2}^{0}\right)-m\left(\tilde{\chi}_{1}^{0}\right)=130 \mathrm{GeV}$, and for a fixed value of the $\tilde{\chi}_{1}^{0}$ mass, respectively.

A summary of the exclusion contours from the analyses presented here is shown in Fig. 14. Observed and expected contours as obtained from each channel are shown, with the exception of the $3 \ell$ analysis, which has no sensitivity. The overall expected sensitivity varies from $m\left(\tilde{\chi}_{1}^{ \pm} / \tilde{\chi}_{2}^{0}\right)=$ $150 \mathrm{GeV}$ to $m\left(\tilde{\chi}_{1}^{ \pm} / \tilde{\chi}_{2}^{0}\right)=635 \mathrm{GeV}$, including significant improvements compared to previous results towards large $m\left(\tilde{\chi}_{1}^{0}\right)$ masses near the kinematic limit of the processes considered. The gain in sensitivity is largely due to the increased center-of-mass energy and dataset size relative to Run 1, the improvements in the optimization of the signal and control region definitions, as well as the addition of the $0 \ell b \bar{b}$ analysis channel.

\section{CONCLUSION}

Results of a comprehensive search for the electroweak pair production of a chargino and a neutralino $p p \rightarrow \tilde{\chi}_{1}^{ \pm} \tilde{\chi}_{2}^{0}$ are presented, based on $36.1 \mathrm{fb}^{-1}$ of proton-proton collision data collected at $\sqrt{s}=13 \mathrm{TeV}$ by the ATLAS experiment at the Large Hadron Collider. Final states are considered where the chargino decays into the lightest neutralino and a $W$ boson, $\tilde{\chi}_{1}^{ \pm} \rightarrow \tilde{\chi}_{1}^{0} W^{ \pm}$, while the next-tolightest neutralino decays into the lightest neutralino and a SM-like $125 \mathrm{GeV}$ Higgs boson, $\tilde{\chi}_{2}^{0} \rightarrow \tilde{\chi}_{1}^{0} h$. The search includes $0 \ell b \bar{b}, 1 \ell b \bar{b}, 1 \ell \gamma \gamma$ and multilepton final states with large missing transverse momentum in order to maximize sensitivity to signals of new physics processes involving $W h$ and SUSY DM candidates. The searches based on final states containing $b$-jets $(0 \ell b \bar{b}$ and $1 \ell b \bar{b})$ provide unprecedented sensitivity to high-mass electroweak production for this benchmark scenario. The multilepton and $1 \ell \gamma \gamma$ searches provide sensitivity in the region of low masses, which is more difficult to access. Crucially, exploiting the various branching ratios of the Higgs boson into bottom quarks, photons, and multileptons, and designing an overall strategy that benefits from the complementarity of the various search channels is essential for the wide sensitivity of this analysis. No evidence of new physics processes is observed and stringent limits are placed on the existence of electroweak production of SUSY particle pairs with significant improvements over previous searches for high $\tilde{\chi}_{1}^{ \pm} \tilde{\chi}_{2}^{0}$ masses. In the context of the considered SUSY model, masses of $\tilde{\chi}_{1}^{ \pm}$and $\tilde{\chi}_{2}^{0}$ smaller than $680 \mathrm{GeV}$ are excluded at $95 \%$ confidence level for a massless neutralino.

\section{ACKNOWLEDGMENTS}

We thank CERN for the very successful operation of the LHC, as well as the support staff from our institutions 
without whom ATLAS could not be operated efficiently. We acknowledge the support of ANPCyT, Argentina; YerPhI, Armenia; ARC, Australia; BMWFW and FWF, Austria; ANAS, Azerbaijan; SSTC, Belarus; CNPq and FAPESP, Brazil; NSERC, NRC and CFI, Canada; CERN; CONICYT, Chile; CAS, MOST and NSFC, China; COLCIENCIAS, Colombia; MSMT CR, MPO CR and VSC CR, Czech Republic; DNRF and DNSRC, Denmark; IN2P3-CNRS, CEA-DRF/IRFU, France; SRNSFG, Georgia; BMBF, HGF, and MPG, Germany; GSRT, Greece; RGC, Hong Kong SAR, China; ISF and Benoziyo Center, Israel; INFN, Italy; MEXT and JSPS, Japan; CNRST, Morocco; NWO, Netherlands; RCN, Norway; MNiSW and NCN, Poland; FCT, Portugal; MNE/IFA, Romania; MES of Russia and NRC KI, Russian Federation; JINR; MESTD, Serbia; MSSR, Slovakia; ARRS and MIZŠ, Slovenia; DST/NRF, South Africa; MINECO, Spain; SRC and Wallenberg Foundation, Sweden; SERI, SNSF and Cantons of Bern and Geneva, Switzerland; MOST, Taiwan; TAEK, Turkey; STFC,
United Kingdom; DOE and NSF, United States of America. In addition, individual groups and members have received support from BCKDF, CANARIE, CRC and Compute Canada, Canada; COST, ERC, ERDF, Horizon 2020, and Marie Skłodowska-Curie Actions, European Union; Investissements d' Avenir Labex and Idex, ANR, France; DFG and AvH Foundation, Germany; Herakleitos, Thales and Aristeia programmes co-financed by EU-ESF and the Greek NSRF, Greece; BSF-NSF and GIF, Israel; CERCA Programme Generalitat de Catalunya, Spain; The Royal Society and Leverhulme Trust, United Kingdom. The crucial computing support from all WLCG partners is acknowledged gratefully, in particular from CERN, the ATLAS Tier-1 facilities at TRIUMF (Canada), NDGF (Denmark, Norway, Sweden), CC-IN2P3 (France), KIT/ GridKA (Germany), INFN-CNAF (Italy), NL-T1 (Netherlands), PIC (Spain), ASGC (Taiwan), RAL (UK) and BNL (USA), the Tier-2 facilities worldwide and large non-WLCG resource providers. Major contributors of computing resources are listed in Ref. [80].
[1] ATLAS Collaboration, Observation of a new particle in the search for the standard model Higgs boson with the ATLAS detector at the LHC, Phys. Lett. B 716, 1 (2012).

[2] CMS Collaboration, Observation of a new boson at a mass of $125 \mathrm{GeV}$ with the CMS experiment at the LHC, Phys. Lett. B 716, 30 (2012).

[3] ATLAS and CMS Collaborations, Combined Measurement of the Higgs Boson Mass in $p p$ Collisions at $\sqrt{s}=7$ and $8 \mathrm{TeV}$ with the ATLAS and CMS Experiments, Phys. Rev. Lett. 114, 191803 (2015).

[4] ATLAS and CMS Collaborations, Measurements of the Higgs boson production and decay rates and constraints on its couplings from a combined ATLAS and CMS analysis of the LHC $p p$ collision data at $\sqrt{s}=7$ and $8 \mathrm{TeV}$, J. High Energy Phys. 08 (2016) 045.

[5] N. Sakai, Naturalness in supersymmetric GUTS, Z. Phys. C 11, 153 (1981).

[6] S. Dimopoulos, S. Raby, and F. Wilczek, Supersymmetry and the scale of unification, Phys. Rev. D 24, 1681 (1981).

[7] L. E. Ibanez and G. G. Ross, Low-energy predictions in supersymmetric grand unified theories, Phys. Lett. B 105, 439 (1981).

[8] S. Dimopoulos and H. Georgi, Softly broken supersymmetry and SU(5), Nucl. Phys. B193, 150 (1981).

[9] Y. A. Golfand and E. P. Likhtman, Extension of the algebra of poincare group generators and violation of $P$ invariance, Pis'ma Zh. Eksp. Teor. Fiz. 13, 452 (1971) [JETP Lett. 13, 323 (1971)].

[10] D. V. Volkov and V. P. Akulov, Is the neutrino a goldstone particle?, Phys. Lett. 46B, 109 (1973).

[11] J. Wess and B. Zumino, Supergauge transformations in fourdimensions, Nucl. Phys. B70, 39 (1974).
[12] J. Wess and B. Zumino, Supergauge invariant extension of quantum electrodynamics, Nucl. Phys. B78, 1 (1974).

[13] S. Ferrara and B. Zumino, Supergauge invariant Yang-Mills theories, Nucl. Phys. B79, 413 (1974).

[14] A. Salam and J. A. Strathdee, Super-symmetry and nonAbelian gauges, Phys. Lett. B 51, 353 (1974).

[15] H. Goldberg, Constraint on the Photino Mass from Cosmology, Phys. Rev. Lett. 50, 1419 (1983); Erratum 103, 099905 (2009).

[16] J. R. Ellis, J. S. Hagelin, D. V. Nanopoulos, K. A. Olive, and M. Srednicki, Supersymmetric relics from the big bang, Nucl. Phys. B238, 453 (1984).

[17] G. R. Farrar and P. Fayet, Phenomenology of the production, decay, and detection of new hadronic states associated with supersymmetry, Phys. Lett. B 76, 575 (1978).

[18] R. Barbieri and G.F. Giudice, Upper bounds on supersymmetric particle masses, Nucl. Phys. B306, 63 (1988).

[19] B. de Carlos and J.A. Casas, One loop analysis of the electroweak breaking in supersymmetric models and the fine tuning problem, Phys. Lett. B 309, 320 (1993).

[20] P. Fayet, Supersymmetry and Weak, Electromagnetic and Strong Interactions, Phys. Lett. B 64, 159 (1976).

[21] P. Fayet, Spontaneously broken supersymmetric theories of weak, electromagnetic and strong interactions, Phys. Lett. B 69, 489 (1977).

[22] S. P. Das, M. Guchait, and D. P. Roy, Testing SUSY models for the muon g-2 anomaly via chargino-neutralino pair production at the LHC, Phys. Rev. D 90, 055011 (2014).

[23] M. A. Ajaib, B. Dutta, T. Ghosh, I. Gogoladze, and Q. Shafi, Neutralinos and sleptons at the LHC in light of muon $(g-2)_{\mu}$, Phys. Rev. D 92, 075033 (2015). 
[24] M. Chakraborti, U. Chattopadhyay, A. Choudhury, A. Datta, and S. Poddar, Reduced LHC constraints for Higgsino-like heavier electroweakinos, J. High Energy Phys. 11 (2015) 050 .

[25] M. Endo, K. Hamaguchi, S. Iwamoto, and K. Yanagi, Probing minimal SUSY scenarios in the light of muon $g-2$ and dark matter, J. High Energy Phys. 06 (2017) 031.

[26] J. Alwall, P. Schuster, and N. Toro, Simplified models for a first characterization of new physics at the LHC, Phys. Rev. D 79, 075020 (2009).

[27] D. Alves, Simplified models for LHC new physics searches, J. Phys. G 39, 105005 (2012).

[28] ATLAS Collaboration, Search for the electroweak production of supersymmetric particles in $\sqrt{s}=8 \mathrm{TeV} p p$ collisions with the ATLAS detector, Phys. Rev. D 93, 052002 (2016).

[29] CMS Collaboration, Combined search for electroweak production of charginos and neutralinos in proton-proton collisions at $\sqrt{s}=13 \mathrm{TeV}$, J. High Energy Phys. 03 (2018) 160.

[30] ATLAS Collaboration, The ATLAS Experiment at the CERN large hadron collider, J. Instrum. 3, S08003 (2008).

[31] ATLAS Collaboration, ATLAS insertable B-layer technical design report, Report No. ATLAS-TDR-19, 2010, https:// cds.cern.ch/record/1291633; ATLAS insertable B-layer technical design report, Report No. ATLAS-TDR-19ADD-1, 2012, https://cds.cern.ch/record/1451888.

[32] B. Abbott et al., Production and integration of the ATLAS insertable B-layer, J. Instrum. 13, T05008 (2018).

[33] ATLAS Collaboration, Performance of the ATLAS trigger system in 2015, Eur. Phys. J. C 77, 317 (2017).

[34] ATLAS Collaboration, Luminosity determination in $p p$ collisions at $\sqrt{s}=8 \mathrm{TeV}$ using the ATLAS detector at the LHC, Eur. Phys. J. C 76, 653 (2016).

[35] G. Avoni et al., The new LUCID-2 detector for luminosity measurement and monitoring in ATLAS, J. Instrum. 13, P07017 (2018).

[36] ATLAS Collaboration, The ATLAS simulation infrastructure, Eur. Phys. J. C 70, 823 (2010).

[37] S. Agostinelli et al., GEANT4 - a simulation toolkit, Nucl. Instrum. Methods Phys. Res., Sect. A 506, 250 (2003).

[38] J. Alwall, R. Frederix, S. Frixione, V. Hirschi, F. Maltoni, O. Mattelaer, H.-S. Shao, T. Stelzer, P. Torrielli, and M. Zaro, The automated computation of tree-level and next-toleading order differential cross sections, and their matching to parton shower simulations, J. High Energy Phys. 07 (2014) 079.

[39] T. Sjöstrand, S. Mrenna, and P.Z. Skands, A brief introduction to PYTHIA 8.1, Comput. Phys. Commun. 178, 852 (2008).

[40] ATLAS Collaboration, ATLAS Pythia 8 tunes to $7 \mathrm{TeV}$ data, Report No. ATL-PHYS-PUB-2014-021, 2014, https://cds .cern.ch/record/1966419.

[41] L. Lönnblad and S. Prestel, Merging multi-leg NLO matrix elements with parton showers, J. High Energy Phys. 03 (2013) 166.

[42] R. D. Ball et al., Parton distributions with LHC data, Nucl. Phys. B867, 244 (2013).
[43] W. Beenakker, M. Kramer, T. Plehn, M. Spira, and P. M. Zerwas, Stop production at hadron colliders, Nucl. Phys. B515, 3 (1998).

[44] W. Beenakker, S. Brensing, M. Krämer, A. Kulesza, E. Laenen, and I. Niessen, Supersymmetric top and bottom squark production at hadron colliders, J. High Energy Phys. 08 (2010) 098.

[45] W. Beenakker, S. Brensing, M. Krämer, A. Kulesza, E. Laenen, L. Motyka, and I. Niessen, Squark and Gluino Hadroproduction, Int. J. Mod. Phys. A 26, 2637 (2011).

[46] C. Borschensky, M. Krämer, A. Kulesza, M. Mangano, S. Padhi, T. Plehn, and X. Portell, Squark and gluino production cross sections in pp collisions at $\sqrt{s}=13,14,33$ and 100 TeV, Eur. Phys. J. C 74, 3174 (2014).

[47] T. Gleisberg, S. Höche, F. Krauss, M. Schönherr, S. Schumann, F. Siegert, and J. Winter, Event generation with SHERPA 1.1, J. High Energy Phys. 02 (2009) 007.

[48] D. J. Lange, The EvtGen particle decay simulation package, Nucl. Instrum. Methods Phys. Res., Sect. A 462, 152 (2001).

[49] S. Alioli, P. Nason, C. Oleari, and E. Re, A general framework for implementing NLO calculations in shower Monte Carlo programs: The POWHEG BOX, J. High Energy Phys. 06 (2010) 043.

[50] S. Frixione, P. Nason, and C. Oleari, Matching NLO QCD computations with parton shower simulations: The POWHEG method, J. High Energy Phys. 11 (2007) 070.

[51] P. Z. Skands, Tuning Monte Carlo generators: The Perugia tunes, Phys. Rev. D 82, 074018 (2010).

[52] H.-L. Lai, M. Guzzi, J. Huston, Z. Li, P. M. Nadolsky, J. Pumplin, and C.-P. Yuan, New parton distributions for collider physics, Phys. Rev. D 82, 074024 (2010).

[53] T. Sjöstrand, S. Mrenna, and P.Z. Skands, PYTHIA 6.4 physics and manual, J. High Energy Phys. 05 (2006) 026.

[54] S. Gieseke, C. Rohr, and A. Siodmok, Colour reconnections in Herwig++, Eur. Phys. J. C 72, 2225 (2012).

[55] ATLAS Collaboration, Vertex reconstruction performance of the ATLAS detector at $\sqrt{s}=13 \mathrm{TeV}$, Report No. ATLPHYS-PUB-2015-026, 2015, https://cds.cern.ch/record/ 2037717.

[56] ATLAS Collaboration, Selection of jets produced in $13 \mathrm{TeV}$ proton-proton collisions with the ATLAS detector, Report No. ATLAS-CONF-2015-029, 2015, https://cds.cern.ch/ record/2037702.

[57] ATLAS Collaboration, Electron and photon energy calibration with the ATLAS detector using 2015-2016 LHC proton-proton collision data, J. Instrum. 14, P03017 (2019).

[58] ATLAS Collaboration, Electron efficiency measurements with the ATLAS detector using the 2015 LHC protonproton collision data, Report No. ATLAS-CONF-2016-024, 2016, https://cds.cern.ch/record/2157687.

[59] ATLAS Collaboration, Measurement of the photon identification efficiencies with the ATLAS detector using LHC run-1 data, Eur. Phys. J. C 76, 666 (2016).

[60] ATLAS Collaboration, Measurement of the photon identification efficiencies with the ATLAS detector using LHC run 2 data collected in 2015 and 2016, Eur. Phys. J. C 79, 205 (2019). 
[61] ATLAS Collaboration, Muon reconstruction performance of the ATLAS detector in proton-proton collision data at $\sqrt{s}=13$ TeV, Eur. Phys. J. C 76, 292 (2016).

[62] ATLAS Collaboration, Topological cell clustering in the ATLAS calorimeters and its performance in LHC run 1, Eur. Phys. J. C 77, 490 (2017).

[63] M. Cacciari, G. P. Salam, and G. Soyez, The anti- $k_{t}$ jet clustering algorithm, J. High Energy Phys. 04 (2008) 063.

[64] ATLAS Collaboration, Jet energy scale measurements and their systematic uncertainties in proton-proton collisions at $\sqrt{s}=13 \mathrm{TeV}$ with the ATLAS detector, Phys. Rev. D 96, 072002 (2017).

[65] ATLAS Collaboration, Performance of pile-up mitigation techniques for jets in $p p$ collisions at $\sqrt{s}=$ $8 \mathrm{TeV}$ using the ATLAS detector, Eur. Phys. J. C 76, 581 (2016).

[66] ATLAS Collaboration, Performance of b-jet identification in the ATLAS experiment, J. Instrum. 11, P04008 (2016).

[67] ATLAS Collaboration, Optimisation of the ATLAS b-tagging performance for the 2016 LHC Run, Report No. ATLPHYS-PUB-2016-012， 2016, https://cds.cern.ch/record/ 2160731.

[68] ATLAS Collaboration, Measurements of $b$-jet tagging efficiency with the ATLAS detector using $t \bar{t}$ events at $\sqrt{s}=13$ TeV, J. High Energy Phys. 08 (2018) 089.

[69] ATLAS Collaboration, Performance of missing transverse momentum reconstruction with the ATLAS detector using proton-proton collisions at $\sqrt{s}=13 \mathrm{TeV}$, Eur. Phys. J. C 78, 903 (2018).

[70] D. R. Tovey, On measuring the masses of pair-produced semi-invisibly decaying particles at hadron colliders, J. High Energy Phys. 04 (2008) 034.
[71] G. Polesello and D. R. Tovey, Supersymmetric particle mass measurement with the boost-corrected contransverse mass, J. High Energy Phys. 03 (2010) 030.

[72] C. G. Lester and D. J. Summers, Measuring masses of semiinvisibly decaying particles pair produced at hadron colliders, Phys. Lett. B 463, 99 (1999).

[73] A. Barr, C. Lester, and P. Stephens, A variable for measuring masses at hadron colliders when missing energy is expected; $m_{T 2}$ : The truth behind the glamour, J. Phys. G 29, 2343 (2003).

[74] M. Baak, G. J. Besjes, D. Côté, A. Koutsman, J. Lorenz, and D. Short, HistFitter software framework for statistical data analysis, Eur. Phys. J. C 75, 153 (2015).

[75] D. de Florian et al., Handbook of LHC Higgs cross sections: 4. Deciphering the nature of the Higgs sector, Report No. CERN-2017-002-M, 2016, https://e-publishing.cern .ch/index.php/CYRM/issue/view/32.

[76] ATLAS Collaboration, Measurement of the top quark-pair production cross section with ATLAS in $p p$ collisions at $\sqrt{s}=7$ TeV, Eur. Phys. J. C 71, 1577 (2011).

[77] ATLAS Collaboration, Estimation of non-prompt and fake lepton backgrounds in final states with top quarks produced in proton-proton collisions at $\sqrt{s}=8 \mathrm{TeV}$ with the ATLAS Detector, Report No. ATLAS-CONF-2014-058, 2014, https://cds.cern.ch/record/1951336.

[78] T. Junk, Confidence level computation for combining searches with small statistics, Nucl. Instrum. Methods Phys. Res., Sect. A 434, 435 (1999).

[79] A. L. Read, Presentation of search results: The CLS technique, J. Phys. G 28, 2693 (2002).

[80] ATLAS Collaboration, ATLAS computing acknowledgements, Report No. ATL-GEN-PUB-2016-002, https://cds .cern.ch/record/2202407.

M. Aaboud ${ }^{35 \mathrm{~d}}$ G. Aad,${ }^{100}$ B. Abbott, ${ }^{127}$ O. Abdinov, ${ }^{13, \mathrm{a}}$ B. Abeloos, ${ }^{131}$ D. K. Abhayasinghe, ${ }^{92}$ S. H. Abidi, ${ }^{166}$ O. S. AbouZeid, ${ }^{40}$ N. L. Abraham, ${ }^{155}$ H. Abramowicz, ${ }^{160}$ H. Abreu, ${ }^{159}$ Y. Abulaiti, ${ }^{6}$ B. S. Acharya,${ }^{65 a, 65 b, b}$ S. Adachi, ${ }^{162}$ L. Adam, ${ }^{98}$ C. Adam Bourdarios, ${ }^{131}$ L. Adamczyk, ${ }^{82 \mathrm{a}}$ J. Adelman, ${ }^{120}$ M. Adersberger, ${ }^{113}$ A. Adiguzel, ${ }^{12 \mathrm{c}}$ T. Adye, ${ }^{143}$ A. A. Affolder, ${ }^{145}$ Y. Afik, ${ }^{159}$ C. Agheorghiesei, ${ }^{27 c}$ J. A. Aguilar-Saavedra, ${ }^{139 f, 139 a}$ F. Ahmadov, ${ }^{78, c}$ G. Aielli, ${ }^{72 a, 72 b}$ S. Akatsuka, ${ }^{84}$ T. P. A. Åkesson, ${ }^{95}$ E. Akilli, ${ }^{53}$ A. V. Akimov, ${ }^{109}$ G. L. Alberghi, ${ }^{23 b, 23 a}$ J. Albert, ${ }^{175}$ P. Albicocco, ${ }^{50}$ M. J. Alconada Verzini, ${ }^{87}$ S. Alderweireldt, ${ }^{118}$ M. Aleksa, ${ }^{36}$ I. N. Aleksandrov ${ }^{78}$ C. Alexa, ${ }^{27 b}$ D. Alexandre, ${ }^{19}$ T. Alexopoulos, ${ }^{10}$ M. Alhroob, ${ }^{127}$ B. Ali,${ }^{141}$ G. Alimonti, ${ }^{67 a}$ J. Alison, ${ }^{37}$ S. P. Alkire, ${ }^{147}$ C. Allaire, ${ }^{131}$ B. M. M. Allbrooke,${ }^{155}$

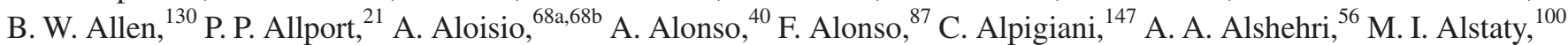
B. Alvarez Gonzalez ${ }^{36}$ D. Álvarez Piqueras, ${ }^{173}$ M. G. Alviggi ${ }^{68 a, 68 b}$ B. T. Amadio, ${ }^{18}$ Y. Amaral Coutinho, ${ }^{79 b}$ A. Ambler, ${ }^{102}$ L. Ambroz,${ }^{134}$ C. Amelung, ${ }^{26}$ D. Amidei, ${ }^{104}$ S. P. Amor Dos Santos, ${ }^{139 a, 139 c}$ S. Amoroso, ${ }^{45}$ C. S. Amrouche, ${ }^{53}$ F. An, ${ }^{77}$ C. Anastopoulos, ${ }^{148}$ L. S. Ancu, ${ }^{53}$ N. Andari, ${ }^{144}$ T. Andeen, ${ }^{11}$ C. F. Anders, ${ }^{60 b}$ J. K. Anders, ${ }^{20}$ K. J. Anderson, ${ }^{37}$ A. Andreazza, ${ }^{67 a, 67 b}$ V. Andrei, ${ }^{60 \mathrm{a}}$ C. R. Anelli, ${ }^{175}$ S. Angelidakis,${ }^{38}$ I. Angelozzi, ${ }^{119}$ A. Angerami, ${ }^{39}$ A. V. Anisenkov, ${ }^{121 b, 121 a}$ A. Annovi ${ }^{70 a}$ C. Antel, ${ }^{60 a}$ M. T. Anthony, ${ }^{148}$ M. Antonelli, ${ }^{50}$ D. J. A. Antrim, ${ }^{170}$ F. Anulli, ${ }^{71 a}$ M. Aoki,${ }^{80}$ J. A. Aparisi Pozo, ${ }^{173}$ L. Aperio Bella, ${ }^{36}$ G. Arabidze, ${ }^{105}$ J. P. Araque, ${ }^{139 a}$ V. Araujo Ferraz ${ }^{79 b}$ R. Araujo Pereira, ${ }^{79 b}$ A. T. H. Arce, ${ }^{48}$ R. E. Ardell, ${ }^{92}$ F. A. Arduh, ${ }^{87}$ J-F. Arguin, ${ }^{108}$ S. Argyropoulos, ${ }^{76}$ J.-H. Arling, ${ }^{45}$ A. J. Armbruster, ${ }^{36}$ L. J. Armitage, ${ }^{91}$ A. Armstrong, ${ }^{170}$ O. Arnaez, ${ }^{166}$ H. Arnold, ${ }^{119}$ M. Arratia ${ }^{32}$ O. Arslan, ${ }^{24}$ A. Artamonov, ${ }^{110, a}$ G. Artoni, ${ }^{134}$ S. Artz, ${ }^{98}$ S. Asai ${ }^{162}$ N. Asbah, ${ }^{58}$ E. M. Asimakopoulou, ${ }^{171}$ L. Asquith, ${ }^{155}$ K. Assamagan, ${ }^{29}$ R. Astalos, ${ }^{28 a}$ R. J. Atkin, ${ }^{33 a}$ M. Atkinson, ${ }^{172}$ N. B. Atlay, ${ }^{150}$ K. Augsten, ${ }^{141}$ G. Avolio, ${ }^{36}$ R. Avramidou, ${ }^{59 a}$ M. K. Ayoub, ${ }^{15 a}$ A. M. Azoulay, ${ }^{167 b}$ G. Azuelos, ${ }^{108, \mathrm{~d}}$ A. E. Baas, ${ }^{60 \mathrm{a}}$ M. J. Baca, ${ }^{21}$ H. Bachacou, ${ }^{144}$ K. Bachas, ${ }^{66 a, 66 \mathrm{~b}}$ M. Backes, ${ }^{134}$ P. Bagnaia, ${ }^{71 a, 71 \mathrm{~b}}$ 
M. Bahmani, ${ }^{83}$ H. Bahrasemani, ${ }^{151}$ A. J. Bailey, ${ }^{173}$ J. T. Baines, ${ }^{143}$ M. Bajic, ${ }^{40}$ C. Bakalis, ${ }^{10}$ O. K. Baker, ${ }^{182}$ P. J. Bakker, ${ }^{119}$ D. Bakshi Gupta, ${ }^{8}$ S. Balaji, ${ }^{156}$ E. M. Baldin, ${ }^{121 b, 121 a}$ P. Balek, ${ }^{179}$ F. Balli,${ }^{144}$ W. K. Balunas, ${ }^{136}$ J. Balz, ${ }^{98}$ E. Banas, ${ }^{83}$

A. Bandyopadhyay, ${ }^{24}$ S. Banerjee, ${ }^{180, e}$ A. A. E. Bannoura, ${ }^{181}$ L. Barak,${ }^{160}$ W. M. Barbe,${ }^{38}$ E. L. Barberio, ${ }^{103}$

D. Barberis, ${ }^{54,54 \mathrm{a}}$ M. Barbero, ${ }^{100}$ T. Barillari, ${ }^{114}$ M-S. Barisits, ${ }^{36}$ J. Barkeloo, ${ }^{130}$ T. Barklow, ${ }^{152}$ R. Barnea,${ }^{159}$ S. L. Barnes,${ }^{59 c}$

B. M. Barnett, ${ }^{143}$ R. M. Barnett, ${ }^{18}$ Z. Barnovska-Blenessy, ${ }^{59}$ A. Baroncelli, ${ }^{73 a}$ G. Barone, ${ }^{29}$ A. J. Barr, ${ }^{134}$

L. Barranco Navarro, ${ }^{173}$ F. Barreiro, ${ }^{97}$ J. Barreiro Guimarães da Costa, ${ }^{15 a}$ R. Bartoldus, ${ }^{152}$ A. E. Barton,${ }^{88}$ P. Bartos, ${ }^{28 a}$

A. Basalaev, ${ }^{137}$ A. Bassalat, ${ }^{131, f}$ R. L. Bates ${ }^{56}$ S. J. Batista, ${ }^{166}$ S. Batlamous, ${ }^{35 e}$ J. R. Batley ${ }^{32}$ M. Battaglia, ${ }^{145}$

M. Bauce, ${ }^{71 a, 71 b}$ F. Bauer, ${ }^{144}$ K. T. Bauer,${ }^{170}$ H. S. Bawa,${ }^{31, g}$ J. B. Beacham, ${ }^{125}$ T. Beau, ${ }^{135}$ P. H. Beauchemin, ${ }^{169}$ P. Bechtle, ${ }^{24}$ H. C. Beck, ${ }^{52}$ H. P. Beck, ${ }^{20, \mathrm{~h}}$ K. Becker, ${ }^{51}$ M. Becker, ${ }^{98}$ C. Becot, ${ }^{45}$ A. Beddall, ${ }^{12 \mathrm{~d}}$ A. J. Beddall, ${ }^{12 \mathrm{a}}$ V. A. Bednyakov, ${ }^{78}$ M. Bedognetti, ${ }^{119}$ C. P. Bee, ${ }^{154}$ T. A. Beermann, ${ }^{75}$ M. Begalli, ${ }^{79 b}$ M. Begel,${ }^{29}$ A. Behera, ${ }^{154}$ J. K. Behr, ${ }^{45}$ A. S. Bell, ${ }^{93}$ G. Bella, ${ }^{160}$ L. Bellagamba, ${ }^{23 b}$ A. Bellerive, ${ }^{34}$ M. Bellomo, ${ }^{159}$ P. Bellos, ${ }^{9}$ K. Belotskiy, ${ }^{111}$ N. L. Belyaev, ${ }^{111}$ O. Benary, ${ }^{160, a}$ D. Benchekroun, ${ }^{35 a}$ M. Bender, ${ }^{113}$ N. Benekos, ${ }^{10}$ Y. Benhammou, ${ }^{160}$ E. Benhar Noccioli, ${ }^{182}$ J. Benitez, ${ }^{76}$ D. P. Benjamin, ${ }^{6}$ M. Benoit, ${ }^{53}$ J. R. Bensinger, ${ }^{26}$ S. Bentvelsen, ${ }^{119}$ L. Beresford, ${ }^{134}$ M. Beretta,${ }^{50}$ D. Berge,${ }^{45}$ E. Bergeaas Kuutmann, ${ }^{171}$ N. Berger, ${ }^{5}$ B. Bergmann, ${ }^{141}$ L. J. Bergsten, ${ }^{26}$ J. Beringer, ${ }^{18}$ S. Berlendis, ${ }^{7}$ N. R. Bernard, ${ }^{101}$ G. Bernardi, ${ }^{135}$ C. Bernius, ${ }^{152}$ F. U. Bernlochner ${ }^{24}$ T. Berry, ${ }^{92}$ P. Berta, ${ }^{98}$ C. Bertella, ${ }^{15 a}$ G. Bertoli, ${ }^{44 a, 44 b}$ I. A. Bertram, ${ }^{88}$ G. J. Besjes,${ }^{40}$

O. Bessidskaia Bylund, ${ }^{181}$ M. Bessner, ${ }^{45}$ N. Besson, ${ }^{144}$ A. Bethani, ${ }^{99}$ S. Bethke, ${ }^{114}$ A. Betti, ${ }^{24}$ A. J. Bevan, ${ }^{91}$ J. Beyer, ${ }^{114}$

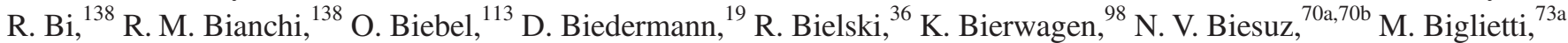
T. R. V. Billoud, ${ }^{108}$ M. Bindi, ${ }^{52}$ A. Bingul, ${ }^{12 \mathrm{~d}}$ C. Bini, ${ }^{71 \mathrm{a}, 71 \mathrm{~b}}$ S. Biondi, ${ }^{23 \mathrm{~b}, 23 \mathrm{a}}$ M. Birman, ${ }^{179}$ T. Bisanz, ${ }^{52}$ J. P. Biswal, ${ }^{160}$ C. Bittrich, ${ }^{47}$ D. M. Bjergaard, ${ }^{48}$ J. E. Black, ${ }^{152}$ K. M. Black,${ }^{25}$ T. Blazek, ${ }^{28 a}$ I. Bloch, ${ }^{45}$ C. Blocker, ${ }^{26}$ A. Blue ${ }^{56}$ U. Blumenschein, ${ }^{91}$ S. Blunier, ${ }^{146 a}$ G. J. Bobbink, ${ }^{119}$ V. S. Bobrovnikov, ${ }^{121 b, 121 a}$ S. S. Bocchetta, ${ }^{95}$ A. Bocci, ${ }^{48}$ D. Boerner, ${ }^{181}$ D. Bogavac, ${ }^{113}$ A. G. Bogdanchikov, ${ }^{121 b, 121 a}$ C. Bohm, ${ }^{44 a}$ V. Boisvert, ${ }^{92}$ P. Bokan, ${ }^{171}$ T. Bold ${ }^{82 a}$ A. S. Boldyrev, ${ }^{112}$

A. E. Bolz, ${ }^{60 b}$ M. Bomben ${ }^{135}$ M. Bona,${ }^{91}$ J. S. Bonilla, ${ }^{130}$ M. Boonekamp, ${ }^{144}$ H. M. Borecka-Bielska, ${ }^{89}$ A. Borisov, ${ }^{122}$ G. Borissov, ${ }^{88}$ J. Bortfeldt, ${ }^{36}$ D. Bortoletto, ${ }^{134}$ V. Bortolotto, ${ }^{72 a, 72 b}$ D. Boscherini, ${ }^{23 b}$ M. Bosman, ${ }^{14}$ J. D. Bossio Sola,${ }^{30}$ K. Bouaouda, ${ }^{35 a}$ J. Boudreau, ${ }^{138}$ E. V. Bouhova-Thacker, ${ }^{88}$ D. Boumediene, ${ }^{38}$ S. K. Boutle, ${ }^{56}$ A. Boveia, ${ }^{125}$ J. Boyd ${ }^{36}$ D. Boye, ${ }^{33 b}$ I. R. Boyko, ${ }^{78}$ A. J. Bozson, ${ }^{92}$ J. Bracinik, ${ }^{21}$ N. Brahimi,,${ }^{100}$ A. Brandt, ${ }^{8}$ G. Brandt, ${ }^{181}$ O. Brandt,${ }^{60 a}$ F. Braren, ${ }^{45}$ U. Bratzler, ${ }^{163}$ B. Brau, ${ }^{101}$ J. E. Brau, ${ }^{130}$ W. D. Breaden Madden, ${ }^{56}$ K. Brendlinger, ${ }^{45}$ L. Brenner ${ }^{45}$ R. Brenner, ${ }^{171}$

S. Bressler, ${ }^{179}$ B. Brickwedde, ${ }^{98}$ D. L. Briglin, ${ }^{21}$ D. Britton, ${ }^{56}$ D. Britzger, ${ }^{114}$ I. Brock,${ }^{24}$ R. Brock, ${ }^{105}$ G. Brooijmans,${ }^{39}$ T. Brooks,${ }^{92}$ W. K. Brooks,${ }^{146 \mathrm{~b}}$ E. Brost,${ }^{120}$ J. H Broughton, ${ }^{21}$ P. A. Bruckman de Renstrom, ${ }^{83}$ D. Bruncko, ${ }^{28 \mathrm{~b}}$ A. Bruni, ${ }^{23 \mathrm{~b}}$ G. Bruni, ${ }^{23 b}$ L. S. Bruni, ${ }^{119}$ S. Bruno, ${ }^{72 a, 72 b}$ B. H. Brunt,${ }^{32}$ M. Bruschi, ${ }^{23 b}$ N. Bruscino, ${ }^{138}$ P. Bryant,${ }^{37}$ L. Bryngemark, ${ }^{95}$ T. Buanes, ${ }^{17}$ Q. Buat, ${ }^{36}$ P. Buchholz, ${ }^{150}$ A. G. Buckley, ${ }^{56}$ I. A. Budagov, ${ }^{78}$ M. K. Bugge, ${ }^{133}$ F. Bührer,${ }^{51}$ O. Bulekov, ${ }^{111}$ D. Bullock, ${ }^{8}$ T. J. Burch ${ }^{120}$ S. Burdin, ${ }^{89}$ C. D. Burgard, ${ }^{119}$ A. M. Burger, ${ }^{5}$ B. Burghgrave, ${ }^{120}$ K. Burka, ${ }^{83}$ S. Burke, ${ }^{143}$

I. Burmeister, ${ }^{46}$ J. T. P. Burr, ${ }^{134}$ V. Büscher, ${ }^{98}$ E. Buschmann, ${ }^{52}$ P. J. Bussey,${ }^{56}$ J. M. Butler, ${ }^{25}$ C. M. Buttar, ${ }^{56}$

J. M. Butterworth, ${ }^{93}$ P. Butti, ${ }^{36}$ W. Buttinger, ${ }^{36}$ A. Buzatu, ${ }^{157}$ A. R. Buzykaev, ${ }^{121 b, 121 a}$ G. Cabras, ${ }^{23 b, 23 a}$ S. Cabrera Urbán, ${ }^{173}$ D. Caforio, ${ }^{141}$ H. Cai,${ }^{172}$ V. M. M. Cairo, ${ }^{2}$ O. Cakir, ${ }^{4 a}$ N. Calace,${ }^{53}$ P. Calafiura, ${ }^{18}$ A. Calandri, ${ }^{100}$ G. Calderini, ${ }^{135}$ P. Calfayan, ${ }^{64}$ G. Callea, ${ }^{56}$ L. P. Caloba,${ }^{79 b}$ S. Calvente Lopez,${ }^{97}$ D. Calvet, ${ }^{38}$ S. Calvet,${ }^{38}$ T. P. Calvet, ${ }^{154}$ M. Calvetti, ${ }^{70 a, 70 b}$ R. Camacho Toro, ${ }^{135}$ S. Camarda, ${ }^{36}$ D. Camarero Munoz, ${ }^{97}$ P. Camarri, ${ }^{72 a, 72 b}$ D. Cameron, ${ }^{133}$ R. Caminal Armadans, ${ }^{101}$ C. Camincher, ${ }^{36}$ S. Campana, ${ }^{36}$ M. Campanelli, ${ }^{93}$ A. Camplani, ${ }^{40}$ A. Campoverde, ${ }^{150}$ V. Canale, ${ }^{68 a, 68 b}$ M. Cano Bret, ${ }^{59 \mathrm{c}}$ J. Cantero, ${ }^{128}$ T. Cao, ${ }^{160}$ Y. Cao, ${ }^{172}$ M. D. M. Capeans Garrido, ${ }^{36}$ I. Caprini, ${ }^{27 b}$ M. Caprini, ${ }^{27 b}$ M. Capua, ${ }^{41 b, 41 a}$ R. M. Carbone ${ }^{39}$ R. Cardarelli, ${ }^{72 a}$ F. C. Cardillo, ${ }^{148}$ I. Carli, ${ }^{142}$ T. Carli,${ }^{36}$ G. Carlino, ${ }^{68 a}$ B. T. Carlson, ${ }^{138}$ L. Carminati, ${ }^{67 a, 67 b}$ R. M. D. Carney, ${ }^{44 a, 44 b}$ S. Caron, ${ }^{118}$ E. Carquin, ${ }^{146 b}$ S. Carrá, ${ }^{67,67 b}$ J. W. S. Carter, ${ }^{166}$ D. Casadei, ${ }^{33 b}$ M. P. Casado, ${ }^{14, i}$ A. F. Casha, ${ }^{166}$ D. W. Casper, ${ }^{170}$ R. Castelijn, ${ }^{119}$ F. L. Castillo, ${ }^{173}$ V. Castillo Gimenez, ${ }^{173}$ N. F. Castro, ${ }^{139 a, 139 e}$ A. Catinaccio, ${ }^{36}$ J. R. Catmore, ${ }^{133}$ A. Cattai,${ }^{36}$ J. Caudron,${ }^{24}$ V. Cavaliere,${ }^{29}$ E. Cavallaro, ${ }^{14}$ D. Cavalli, ${ }^{67 a}$ M. Cavalli-Sforza, ${ }^{14}$ V. Cavasinni, ${ }^{70 a, 70 b}$ E. Celebi, ${ }^{12 b}$ F. Ceradini, ${ }^{73 a, 73 b}$ L. Cerda Alberich, ${ }^{173}$ A. S. Cerqueira ${ }^{79 a}$ A. Cerri, ${ }^{155}$ L. Cerrito, ${ }^{72 a, 72 b}$ F. Cerutti, ${ }^{18}$ A. Cervelli, ${ }^{23 b, 23 a}$ S. A. Cetin, ${ }^{12 b}$ A. Chafaq, ${ }^{35 a}$ D. Chakraborty, ${ }^{120}$ S. K. Chan, ${ }^{58}$ W. S. Chan, ${ }^{119}$ Y. L. Chan, ${ }^{62 \mathrm{a}}$ J. D. Chapman, ${ }^{32}$ B. Chargeishvili, ${ }^{158 \mathrm{~b}}$ D. G. Charlton, ${ }^{21}$ C. C. Chau, ${ }^{34}$ C. A. Chavez Barajas, ${ }^{155}$ S. Che, ${ }^{125}$ A. Chegwidden, ${ }^{105}$ S. Chekanov, ${ }^{6}$ S. V. Chekulaev, ${ }^{167 a}$ G. A. Chelkov, ${ }^{78, j}$ M. A. Chelstowska, ${ }^{36}$ C. Chen,${ }^{59 a}$ C. H. Chen, ${ }^{77}$ H. Chen, ${ }^{29}$ J. Chen, ${ }^{59 a}$ J. Chen, ${ }^{39}$ S. Chen, ${ }^{136}$ S. J. Chen, ${ }^{15 \mathrm{c}}$ X. Chen, ${ }^{15 b, k}$ Y. Chen, ${ }^{81}$ Y-H. Chen, ${ }^{45}$ H. C. Cheng, ${ }^{104}$

H. J. Cheng, ${ }^{15 a, 15 d}$ A. Cheplakov, ${ }^{78}$ E. Cheremushkina, ${ }^{122}$ R. Cherkaoui El Moursli, ${ }^{35 e}$ E. Cheu, ${ }^{7}$ K. Cheung, ${ }^{63}$

T. J. A. Chevalérias, ${ }^{144}$ L. Chevalier, ${ }^{144}$ V. Chiarella, ${ }^{50}$ G. Chiarelli, ${ }^{70 a}$ G. Chiodini, ${ }^{66 a}$ A. S. Chisholm, ${ }^{36,21}$ A. Chitan, ${ }^{27 b}$ 
I. Chiu, ${ }^{162}$ Y. H. Chiu, ${ }^{175}$ M. V. Chizhov, ${ }^{78}$ K. Choi, ${ }^{64}$ A. R. Chomont, ${ }^{131}$ S. Chouridou, ${ }^{161}$ Y. S. Chow, ${ }^{119}$ V. Christodoulou, ${ }^{93}$ M. C. Chu, ${ }^{62 a}$ J. Chudoba, ${ }^{140}$ A. J. Chuinard, ${ }^{102}$ J. J. Chwastowski, ${ }^{83}$ L. Chytka, ${ }^{129}$ D. Cinca, ${ }^{46}$ V. Cindro, ${ }^{90}$ I. A. Cioară, ${ }^{24}$ A. Ciocio, ${ }^{18}$ F. Cirotto, ${ }^{68 a, 68 b}$ Z. H. Citron, ${ }^{179}$ M. Citterio, ${ }^{67 a}$ A. Clark, ${ }^{53}$ M. R. Clark, ${ }^{39}$

P. J. Clark, ${ }^{49}$ C. Clement, ${ }^{44 a, 44 b}$ Y. Coadou, ${ }^{100}$ M. Cobal, ${ }^{65 a, 65 c}$ A. Coccaro,${ }^{54 b}$ J. Cochran, ${ }^{77}$ H. Cohen, ${ }^{160}$ A. E. C. Coimbra, ${ }^{179}$ L. Colasurdo,${ }^{118}$ B. Cole,${ }^{39}$ A. P. Colijn, ${ }^{119}$ J. Collot,${ }^{57}$ P. Conde Muiño, ${ }^{139 a}$ E. Coniavitis, ${ }^{51}$ S. H. Connell, ${ }^{33 \mathrm{~b}}$ I. A. Connelly, ${ }^{99}$ S. Constantinescu, ${ }^{27 \mathrm{~b}}$ F. Conventi, ${ }^{68,1}$ A. M. Cooper-Sarkar, ${ }^{134}$ F. Cormier, ${ }^{174}$ K. J. R. Cormier, ${ }^{166}$ L. D. Corpe,${ }^{93}$ M. Corradi, ${ }^{71 a, 71 b}$ E. E. Corrigan, ${ }^{95}$ F. Corriveau, ${ }^{102, \mathrm{~m}}$ A. Cortes-Gonzalez, ${ }^{36}$ M. J. Costa, ${ }^{173}$ F. Costanza, ${ }^{5}$ D. Costanzo, ${ }^{148}$ G. Cottin, ${ }^{32}$ G. Cowan, ${ }^{92}$ B. E. Cox,${ }^{99}$ J. Crane, ${ }^{99}$ K. Cranmer, ${ }^{123}$ S. J. Crawley, ${ }^{56}$ R. A. Creager, ${ }^{136}$ G. Cree,${ }^{34}$ S. Crépé-Renaudin, ${ }^{57}$ F. Crescioli, ${ }^{135}$ M. Cristinziani, ${ }^{24}$ V. Croft, ${ }^{123}$ G. Crosetti, ${ }^{41 b, 41 a}$ A. Cueto, ${ }^{97}$ T. Cuhadar Donszelmann, ${ }^{148}$ A. R. Cukierman, ${ }^{152}$ S. Czekierda, ${ }^{83}$ P. Czodrowski, ${ }^{36}$ M. J. Da Cunha Sargedas De Sousa, ${ }^{59 b}$ C. Da Via,${ }^{99}$ W. Dabrowski, ${ }^{82 a}$ T. Dado, ${ }^{28 a, n}$ S. Dahbi, ${ }^{35 e}$ T. Dai,${ }^{104}$ F. Dallaire, ${ }^{108}$ C. Dallapiccola, ${ }^{101}$ M. Dam, ${ }^{40}$ G. D'amen, ${ }^{23 b, 23 a}$ J. Damp, ${ }^{98}$ J. R. Dandoy, ${ }^{136}$ M. F. Daneri, ${ }^{30}$ N. P. Dang, ${ }^{180}$ N. D Dann, ${ }^{99}$ M. Danninger, ${ }^{174}$ V. Dao,${ }^{36}$ G. Darbo, ${ }^{54 b}$ S. Darmora, ${ }^{8}$ O. Dartsi, ${ }^{5}$ A. Dattagupta, ${ }^{130}$ T. Daubney, ${ }^{45}$ S. D'Auria, ${ }^{67 a, 67 b}$

W. Davey, ${ }^{24}$ C. David, ${ }^{45}$ T. Davidek, ${ }^{142}$ D. R. Davis, ${ }^{48}$ E. Dawe, ${ }^{103}$ I. Dawson,${ }^{148}$ K. De,${ }^{8}$ R. De Asmundis,${ }^{68 a}$ A. De Benedetti, ${ }^{127}$ M. De Beurs, ${ }^{119}$ S. De Castro, ${ }^{23 b, 23 a}$ S. De Cecco, ${ }^{71 a, 71 b}$ N. De Groot, ${ }^{118}$ P. de Jong, ${ }^{119}$ H. De la Torre,${ }^{105}$ F. De Lorenzi, ${ }^{77}$ A. De Maria $,{ }^{70 a}, 70 \mathrm{~b}$ D. De Pedis, ${ }^{71 a}$ A. De Salvo, ${ }^{71 \mathrm{a}}$ U. De Sanctis,${ }^{7 \mathrm{a}, 72 \mathrm{~b}}$ M. De Santis, ${ }^{72 a, 72 \mathrm{~b}}$ A. De Santo, ${ }^{155}$

K. De Vasconcelos Corga ${ }^{100}$ J. B. De Vivie De Regie, ${ }^{131}$ C. Debenedetti, ${ }^{145}$ D. V. Dedovich ${ }^{78}$ N. Dehghanian, ${ }^{3}$ A. M. Deiana, ${ }^{104}$ M. Del Gaudio, ${ }^{41 b, 41 a}$ J. Del Peso, ${ }^{97}$ Y. Delabat Diaz,${ }^{45}$ D. Delgove, ${ }^{131}$ F. Deliot, ${ }^{144}$ C. M. Delitzsch, ${ }^{7}$ M. Della Pietra, ${ }^{68 a, 68 b}$ D. Della Volpe,${ }^{53}$ A. Dell'Acqua, ${ }^{36}$ L. Dell'Asta, ${ }^{25}$ M. Delmastro, ${ }^{5}$ C. Delporte,${ }^{131}$ P. A. Delsart, ${ }^{57}$ D. A. DeMarco, ${ }^{166}$ S. Demers, ${ }^{182}$ M. Demichev, ${ }^{78}$ S. P. Denisov, ${ }^{122}$ D. Denysiuk, ${ }^{119}$ L. D'Eramo, ${ }^{135}$ D. Derendarz, ${ }^{83}$ J. E. Derkaoui, ${ }^{35 d}$ F. Derue, ${ }^{135}$ P. Dervan, ${ }^{89}$ K. Desch,${ }^{24}$ C. Deterre, ${ }^{45}$ K. Dette, ${ }^{166}$ M. R. Devesa,${ }^{30}$ P. O. Deviveiros, ${ }^{36}$

A. Dewhurst, ${ }^{143}$ S. Dhaliwal, ${ }^{26}$ F. A. Di Bello, ${ }^{53}$ A. Di Ciaccio, ${ }^{72 a, 72 b}$ L. Di Ciaccio, ${ }^{5}$ W. K. Di Clemente, ${ }^{136}$

C. Di Donato, ${ }^{68 a, 68 b}$ A. Di Girolamo, ${ }^{36}$ G. Di Gregorio, ${ }^{70 a, 70 b}$ B. Di Micco, ${ }^{73 a, 73 b}$ R. Di Nardo, ${ }^{101}$ K. F. Di Petrillo, ${ }^{58}$

R. Di Sipio ${ }^{166}$ D. Di Valentino, ${ }^{34}$ C. Diaconu, ${ }^{100}$ M. Diamond, ${ }^{166}$ F. A. Dias,${ }^{40}$ T. Dias Do Vale, ${ }^{139 a}$ M. A. Diaz, ${ }^{146 a}$ J. Dickinson, ${ }^{18}$ E. B. Diehl, ${ }^{104}$ J. Dietrich, ${ }^{19}$ S. Díez Cornell, ${ }^{45}$ A. Dimitrievska, ${ }^{18}$ J. Dingfelder, ${ }^{24}$ F. Dittus,${ }^{36}$ F. Djama, ${ }^{100}$ T. Djobava, ${ }^{158 \mathrm{~b}}$ J. I. Djuvsland, ${ }^{60 \mathrm{a}}$ M. A. B. Do Vale ${ }^{79 \mathrm{c}}$ M. Dobre, ${ }^{27 \mathrm{~b}}$ D. Dodsworth, ${ }^{26}$ C. Doglioni, ${ }^{95}$ J. Dolejsi, ${ }^{142}$ Z. Dolezal, ${ }^{142}$ M. Donadelli, ${ }^{79 d}$ J. Donini, ${ }^{38}$ A. D'onofrio, ${ }^{91}$ M. D'Onofrio, ${ }^{89}$ J. Dopke, ${ }^{143}$ A. Doria, ${ }^{68 a}$ M. T. Dova,${ }^{87}$ A. T. Doyle,${ }^{56}$ E. Drechsler, ${ }^{52}$ E. Dreyer, ${ }^{151}$ T. Dreyer, ${ }^{52}$ Y. Du, ${ }^{59}$ F. Dubinin, ${ }^{109}$ M. Dubovsky, ${ }^{28 a}$ A. Dubreuil, ${ }^{53}$ E. Duchovni, ${ }^{179}$ G. Duckeck, ${ }^{113}$ A. Ducourthial, ${ }^{135}$ O. A. Ducu, ${ }^{108,0}$ D. Duda, ${ }^{114}$ A. Dudarev, ${ }^{36}$ A. C. Dudder,${ }^{98}$ E. M. Duffield, ${ }^{18}$ L. Duflot, ${ }^{131}$ M. Dührssen, ${ }^{36}$ C. Dülsen, ${ }^{181}$ M. Dumancic, ${ }^{179}$ A. E. Dumitriu, ${ }^{27 b, p}$ A. K. Duncan,${ }^{56}$ M. Dunford, ${ }^{60 a}$ A. Duperrin, ${ }^{100}$ H. Duran Yildiz, ${ }^{4 \mathrm{a}}$ M. Düren,${ }^{55}$ A. Durglishvili, ${ }^{158 \mathrm{~b}}$ D. Duschinger ${ }^{47}$ B. Dutta, ${ }^{45}$ D. Duvnjak, ${ }^{1}$ G. I. Dyckes, ${ }^{136}$ M. Dyndal, ${ }^{45}$ S. Dysch, ${ }^{99}$ B. S. Dziedzic, ${ }^{83}$ C. Eckardt, ${ }^{45}$ K. M. Ecker, ${ }^{114}$ R. C. Edgar, ${ }^{104}$ T. Eifert, ${ }^{36}$ G. Eigen, ${ }^{17}$ K. Einsweiler, ${ }^{18}$ T. Ekelof, ${ }^{171}$ M. El Kacimi, ${ }^{35 c}$ R. El Kosseifi, ${ }^{100}$ V. Ellajosyula, ${ }^{100}$ M. Ellert, ${ }^{171}$ F. Ellinghaus, ${ }^{181}$ A. A. Elliot, ${ }^{91}$ N. Ellis, ${ }^{36}$ J. Elmsheuser, ${ }^{29}$ M. Elsing, ${ }^{36}$ D. Emeliyanov, ${ }^{143}$ A. Emerman, ${ }^{39}$ Y. Enari, ${ }^{162}$ J. S. Ennis, ${ }^{177}$ M. B. Epland, ${ }^{48}$ J. Erdmann, ${ }^{46}$ A. Ereditato, ${ }^{20}$ S. Errede, ${ }^{172}$ M. Escalier,${ }^{131}$ C. Escobar, ${ }^{173}$ O. Estrada Pastor, ${ }^{173}$ A. I. Etienvre, ${ }^{144}$ E. Etzion, ${ }^{160}$ H. Evans, ${ }^{64}$ A. Ezhilov, ${ }^{137}$ M. Ezzi ${ }^{35 e}$ F. Fabbri,${ }^{56}$ L. Fabbri, ${ }^{23 b, 23 a}$ V. Fabiani, ${ }^{118}$ G. Facini, ${ }^{93}$ R. M. Faisca Rodrigues Pereira, ${ }^{139 a}$ R. M. Fakhrutdinov, ${ }^{122}$ S. Falciano, ${ }^{71 a}$ P. J. Falke, ${ }^{5}$ S. Falke, ${ }^{5}$ J. Faltova, ${ }^{142}$ Y. Fang, ${ }^{15 a}$ M. Fanti, ${ }^{67,67 b}$ A. Farbin, ${ }^{8}$ A. Farilla, ${ }^{73 a}$ E. M. Farina,${ }^{69 a, 69 b}$ T. Farooque, ${ }^{105}$ S. Farrell, ${ }^{18}$ S. M. Farrington, ${ }^{177}$ P. Farthouat,${ }^{36}$

F. Fassi, ${ }^{35 \mathrm{e}}$ P. Fassnacht, ${ }^{36}$ D. Fassouliotis, ${ }^{9}$ M. Faucci Giannelli, ${ }^{49}$ A. Favareto, ${ }^{54 b, 54 \mathrm{a}}$ W. J. Fawcett, ${ }^{32}$ L. Fayard, ${ }^{131}$

O. L. Fedin, ${ }^{137, q}$ W. Fedorko, ${ }^{174}$ M. Feickert, ${ }^{42}$ S. Feigl,${ }^{133}$ L. Feligioni, ${ }^{100}$ C. Feng, ${ }^{59 b}$ E. J. Feng, ${ }^{36}$ M. Feng, ${ }^{48}$

M. J. Fenton ${ }^{56}$ A. B. Fenyuk, ${ }^{122}$ L. Feremenga, ${ }^{8}$ J. Ferrando, ${ }^{45}$ A. Ferrari, ${ }^{171}$ P. Ferrari, ${ }^{119}$ R. Ferrari, ${ }^{69 a}$

D. E. Ferreira de Lima, ${ }^{60 \mathrm{~b}}$ A. Ferrer, ${ }^{173}$ D. Ferrere, ${ }^{53}$ C. Ferretti, ${ }^{104}$ F. Fiedler, ${ }^{98}$ A. Filipčič,${ }^{90}$ F. Filthaut, ${ }^{118}$ K. D. Finelli, ${ }^{25}$

M. C. N. Fiolhais ${ }^{139 a, 139 c, r}$ L. Fiorini, ${ }^{173}$ C. Fischer, ${ }^{14}$ W. C. Fisher, ${ }^{105}$ N. Flaschel,${ }^{45}$ I. Fleck, ${ }^{150}$ P. Fleischmann, ${ }^{104}$ R. R. M. Fletcher, ${ }^{136}$ T. Flick, ${ }^{181}$ B. M. Flierl, ${ }^{113}$ L. F. Flores, ${ }^{136}$ L. R. Flores Castillo, ${ }^{62 \mathrm{a}}$ F. M. Follega, ${ }^{74 \mathrm{a}, 74 \mathrm{~b}}$ N. Fomin, ${ }^{17}$ G. T. Forcolin, ${ }^{74 a, 74 b}$ A. Formica, ${ }^{144}$ F. A. Förster, ${ }^{14}$ A. C. Forti, ${ }^{99}$ A. G. Foster, ${ }^{21}$ D. Fournier, ${ }^{131}$ H. Fox ${ }^{88}$ S. Fracchia, ${ }^{148}$ P. Francavilla, ${ }^{70 a, 70 b}$ M. Franchini, ${ }^{23 b, 23 a}$ S. Franchino, ${ }^{60 a}$ D. Francis,${ }^{36}$ L. Franconi, ${ }^{145}$ M. Franklin, ${ }^{58}$ M. Frate, ${ }^{170}$ M. Fraternali, ${ }^{69 a, 69 b}$ A. N. Fray, ${ }^{91}$ D. Freeborn,${ }^{93}$ B. Freund, ${ }^{108}$ W. S. Freund, ${ }^{79 b}$ E. M. Freundlich, ${ }^{46}$ D. C. Frizzell, ${ }^{127}$ D. Froidevaux, ${ }^{36}$ J. A. Frost, ${ }^{134}$ C. Fukunaga, ${ }^{163}$ E. Fullana Torregrosa, ${ }^{173}$ T. Fusayasu, ${ }^{115}$ J. Fuster, ${ }^{173}$ O. Gabizon, ${ }^{159}$ A. Gabrielli, ${ }^{23 b, 23 a}$ A. Gabrielli, ${ }^{18}$ G. P. Gach, ${ }^{82 a}$ S. Gadatsch,${ }^{53}$ P. Gadow, ${ }^{114}$ G. Gagliardi, ${ }^{54,54 a}$ L. G. Gagnon, ${ }^{108}$ 
C. Galea, ${ }^{27 b}$ B. Galhardo, ${ }^{139 a, 139 c}$ G. E. Gallardo, ${ }^{134}$ E. J. Gallas, ${ }^{134}$ B. J. Gallop, ${ }^{143}$ P. Gallus, ${ }^{141}$ G. Galster, ${ }^{40}$ R. Gamboa Goni, ${ }^{91}$ K. K. Gan, ${ }^{125}$ S. Ganguly, ${ }^{179}$ J. Gao, ${ }^{59 a}$ Y. Gao, ${ }^{89}$ Y. S. Gao, ${ }^{31, g}$ C. García, ${ }^{173}$ J. E. García Navarro, ${ }^{173}$ J. A. García Pascual, ${ }^{15 a}$ M. Garcia-Sciveres, ${ }^{18}$ R. W. Gardner, ${ }^{37}$ N. Garelli, ${ }^{152}$ S. Gargiulo, ${ }^{51}$ V. Garonne, ${ }^{133}$ K. Gasnikova, ${ }^{45}$ A. Gaudiello, ${ }^{54 b, 54 a}$ G. Gaudio, ${ }^{69 a}$ I. L. Gavrilenko, ${ }^{109}$ A. Gavrilyuk, ${ }^{110}$ C. Gay, ${ }^{174}$ G. Gaycken, ${ }^{24}$ E. N. Gazis, ${ }^{10}$ C. N. P. Gee, ${ }^{143}$ J. Geisen,${ }^{52}$ M. Geisen, ${ }^{98}$ M. P. Geisler, ${ }^{60 \mathrm{a}}$ K. Gellerstedt, ${ }^{44 a, 44 \mathrm{~b}}$ C. Gemme, ${ }^{5 \mathrm{~b}}$ M. H. Genest, ${ }^{57}$ C. Geng, ${ }^{104}$ S. Gentile, ${ }^{71 \mathrm{a}, 71 \mathrm{~b}}$ S. George, ${ }^{92}$ D. Gerbaudo, ${ }^{14}$ G. Gessner, ${ }^{46}$ S. Ghasemi, ${ }^{150}$ M. Ghasemi Bostanabad, ${ }^{175}$ M. Ghneimat ${ }^{24}$ B. Giacobbe, ${ }^{23 b}$ S. Giagu, ${ }^{71 a, 71 b}$ N. Giangiacomi, ${ }^{23 b, 23 a}$ P. Giannetti, ${ }^{70 a}$ A. Giannini, ${ }^{68,68 b}$ S. M. Gibson, ${ }^{92}$ M. Gignac, ${ }^{145}$ D. Gillberg, ${ }^{34}$ G. Gilles, ${ }^{181}$ D. M. Gingrich, ${ }^{3, d}$ M. P. Giordani, ${ }^{65,65 \mathrm{c}}$ F. M. Giorgi, ${ }^{23 \mathrm{~b}}$ P. F. Giraud, ${ }^{144}$ P. Giromini, ${ }^{58}$ G. Giugliarelli, ${ }^{65 a, 65 c}$ D. Giugni, ${ }^{67 a}$ F. Giuli, ${ }^{134}$ M. Giulini, ${ }^{60 b}$ S. Gkaitatzis, ${ }^{161}$ I. Gkialas, ${ }^{9, s}$ E. L. Gkougkousis, ${ }^{14}$ P. Gkountoumis, ${ }^{10}$ L. K. Gladilin, ${ }^{112}$ C. Glasman, ${ }^{97}$ J. Glatzer, ${ }^{14}$ P. C. F. Glaysher, ${ }^{45}$ A. Glazov, ${ }^{45}$ M. Goblirsch-Kolb,${ }^{26}$ J. Godlewski, ${ }^{83}$ S. Goldfarb, ${ }^{103}$ T. Golling ${ }^{53}$ D. Golubkov, ${ }^{122}$ A. Gomes, ${ }^{139 a, 139 b}$ R. Goncalves Gama, ${ }^{52}$ R. Gonçalo, ${ }^{139 a}$ G. Gonella, ${ }^{51}$ L. Gonella, ${ }^{21}$ A. Gongadze, ${ }^{78}$ F. Gonnella, ${ }^{21}$ J. L. Gonski, ${ }^{58}$ S. González de la Hoz, ${ }^{173}$ S. Gonzalez-Sevilla, ${ }^{53}$ L. Goossens, ${ }^{36}$ P. A. Gorbounov, ${ }^{110}$ H. A. Gordon, ${ }^{29}$ B. Gorini, ${ }^{36}$ E. Gorini,${ }^{66 a, 66 b}$ A. Gorišek, ${ }^{90}$ A. T. Goshaw, ${ }^{48}$ C. Gössling, ${ }^{46}$ M. I. Gostkin, ${ }^{78}$ C. A. Gottardo, ${ }^{24}$ C. R. Goudet, ${ }^{131}$ D. Goujdami, ${ }^{35 c}$ A. G. Goussiou, ${ }^{147}$ N. Govender, ${ }^{33 b, t}$ C. Goy, ${ }^{5}$ E. Gozani, ${ }^{159}$ I. Grabowska-Bold, ${ }^{82 a}$ P. O. J. Gradin, ${ }^{171}$ E. C. Graham, ${ }^{89}$ J. Gramling, ${ }^{170}$ E. Gramstad, ${ }^{133}$ S. Grancagnolo, ${ }^{19}$ V. Gratchev, ${ }^{137}$ P. M. Gravila, ${ }^{27 f}$ F. G. Gravili, ${ }^{66 a, 66 b}$ C. Gray, ${ }^{56}$ H. M. Gray, ${ }^{18}$ Z. D. Greenwood, ${ }^{94}$ C. Grefe, ${ }^{24}$ K. Gregersen, ${ }^{95}$ I. M. Gregor, ${ }^{45}$ P. Grenier, ${ }^{152}$ K. Grevtsov, ${ }^{45}$ N. A. Grieser, ${ }^{127}$ J. Griffiths, ${ }^{8}$ A. A. Grillo, ${ }^{145}$ K. Grimm, ${ }^{31, u}$ S. Grinstein, ${ }^{14, v}$ Ph. Gris, ${ }^{38}$ J.-F. Grivaz, ${ }^{131}$ S. Groh, ${ }^{98}$ E. Gross, ${ }^{179}$ J. Grosse-Knetter, ${ }^{52}$ G. C. Grossi, ${ }^{94}$ Z. J. Grout ${ }^{93}$ C. Grud, ${ }^{104}$ A. Grummer, ${ }^{17}$ L. Guan, ${ }^{104}$ W. Guan, ${ }^{180}$ J. Guenther, ${ }^{36}$ A. Guerguichon, ${ }^{131}$ F. Guescini, ${ }^{167 a}$ D. Guest, ${ }^{170}$ R. Gugel, ${ }^{51}$ B. Gui, ${ }^{125}$ T. Guillemin,,${ }^{5}$ S. Guindon, ${ }^{36}$ U. Gul, ${ }^{56}$ C. Gumpert, ${ }^{36}$ J. Guo, ${ }^{59 c}$ W. Guo, ${ }^{104}$ Y. Guo, ${ }^{59 a, w}$ Z. Guo, ${ }^{100}$ R. Gupta, ${ }^{45}$ S. Gurbuz, ${ }^{12 c}$ G. Gustavino, ${ }^{127}$ B. J. Gutelman, ${ }^{159}$ P. Gutierrez, ${ }^{127}$ C. Gutschow, ${ }^{93}$ C. Guyot, ${ }^{144}$ M. P. Guzik, ${ }^{82 a}$ C. Gwenlan, ${ }^{134}$ C. B. Gwilliam, ${ }^{89}$ A. Haas, ${ }^{123}$ C. Haber, ${ }^{18}$ H. K. Hadavand, ${ }^{8}$ N. Haddad, ${ }^{35 e}$ A. Hadef, ${ }^{59 a}$

S. Hageböck, ${ }^{24}$ M. Hagihara, ${ }^{168}$ H. Hakobyan, ${ }^{183, a}$ M. Haleem, ${ }^{176}$ J. Haley, ${ }^{128}$ G. Halladjian, ${ }^{105}$ G. D. Hallewell, ${ }^{100}$

K. Hamacher, ${ }^{181}$ P. Hamal, ${ }^{129}$ K. Hamano, ${ }^{175}$ A. Hamilton, ${ }^{33 a}$ G. N. Hamity, ${ }^{148}$ K. Han,${ }^{59 a, x}$ L. Han, ${ }^{59 a}$ S. Han, ${ }^{15 a, 15 d}$ K. Hanagaki, ${ }^{80, y}$ M. Hance, ${ }^{145}$ D. M. Handl, ${ }^{113}$ B. Haney, ${ }^{136}$ R. Hankache, ${ }^{135}$ P. Hanke, ${ }^{60 a}$ E. Hansen, ${ }^{95}$ J. B. Hansen, ${ }^{40}$ J. D. Hansen, ${ }^{40}$ M. C. Hansen, ${ }^{24}$ P. H. Hansen, ${ }^{40}$ E. C. Hanson, ${ }^{99}$ K. Hara, ${ }^{168}$ A. S. Hard ${ }^{180}$ T. Harenberg, ${ }^{181}$ S. Harkusha, ${ }^{106}$ P. F. Harrison, ${ }^{177}$ N. M. Hartmann, ${ }^{113}$ Y. Hasegawa, ${ }^{149}$ A. Hasib, ${ }^{49}$ S. Hassani, ${ }^{144}$ S. Haug, ${ }^{20}$ R. Hauser, ${ }^{105}$ L. Hauswald,${ }^{47}$

L. B. Havener, ${ }^{39}$ M. Havranek, ${ }^{141}$ C. M. Hawkes, ${ }^{21}$ R. J. Hawkings,${ }^{36}$ D. Hayden, ${ }^{105}$ C. Hayes,${ }^{154}$ C. P. Hays,${ }^{134}$ J. M. Hays, ${ }^{91}$ H. S. Hayward, ${ }^{89}$ S. J. Haywood, ${ }^{143}$ M. P. Heath, ${ }^{49}$ V. Hedberg, ${ }^{95}$ L. Heelan, ${ }^{8}$ S. Heer, ${ }^{24}$ K. K. Heidegger,${ }^{51}$ J. Heilman, ${ }^{34}$ S. Heim, ${ }^{45}$ T. Heim, ${ }^{18}$ B. Heinemann,${ }^{45, z}$ J. J. Heinrich, ${ }^{113}$ L. Heinrich,${ }^{123}$ C. Heinz, ${ }^{55}$ J. Hejbal, ${ }^{140}$ L. Helary, ${ }^{36}$

A. Held, ${ }^{174}$ S. Hellesund, ${ }^{133}$ C. M. Helling, ${ }^{145}$ S. Hellman, ${ }^{44 a, 44 b}$ C. Helsens,${ }^{36}$ R. C. W. Henderson, ${ }^{88}$ Y. Heng, ${ }^{180}$

S. Henkelmann, ${ }^{174}$ A. M. Henriques Correia, ${ }^{36}$ G. H. Herbert, ${ }^{19}$ H. Herde,${ }^{26}$ V. Herget,${ }^{176}$ Y. Hernández Jiménez, ${ }^{33 c}$

H. Herr, ${ }^{98}$ M. G. Herrmann, ${ }^{113}$ T. Herrmann, ${ }^{47}$ G. Herten, ${ }^{51}$ R. Hertenberger, ${ }^{113}$ L. Hervas, ${ }^{36}$ T. C. Herwig, ${ }^{136}$

G. G. Hesketh, ${ }^{93}$ N. P. Hessey, ${ }^{167 a}$ A. Higashida, ${ }^{162}$ S. Higashino, ${ }^{80}$ E. Higón-Rodriguez, ${ }^{173}$ K. Hildebrand, ${ }^{37}$ E. Hill, ${ }^{175}$ J. C. Hill, ${ }^{32}$ K. K. Hill, ${ }^{29}$ K. H. Hiller, ${ }^{45}$ S. J. Hillier, ${ }^{21}$ M. Hils, ${ }^{47}$ I. Hinchliffe,${ }^{18}$ M. Hirose, ${ }^{132}$ D. Hirschbuehl, ${ }^{181}$ B. Hiti, ${ }^{90}$ O. Hladik, ${ }^{140}$ D. R. Hlaluku, ${ }^{33 \mathrm{c}}$ X. Hoad, ${ }^{49}$ J. Hobbs, ${ }^{154}$ N. Hod, ${ }^{167 a}$ M. C. Hodgkinson, ${ }^{148}$ A. Hoecker, ${ }^{36}$

M. R. Hoeferkamp, ${ }^{117}$ F. Hoenig, ${ }^{113}$ D. Hohn, ${ }^{51}$ D. Hohov, ${ }^{131}$ T. R. Holmes,${ }^{37}$ M. Holzbock, ${ }^{113}$ M. Homann, ${ }^{46}$ L. B. A. H. Hommels, ${ }^{32}$ S. Honda, ${ }^{168}$ T. Honda, ${ }^{80}$ T. M. Hong, ${ }^{138}$ A. Hönle, ${ }^{114}$ B. H. Hooberman, ${ }^{172}$ W. H. Hopkins,${ }^{130}$ Y. Horii, ${ }^{116}$ P. Horn, ${ }^{47}$ A. J. Horton, ${ }^{151}$ L. A. Horyn, ${ }^{37}$ J-Y. Hostachy, ${ }^{57}$ A. Hostiuc, ${ }^{147}$ S. Hou, ${ }^{157}$ A. Hoummada, ${ }^{35 a}$ J. Howarth, ${ }^{99}$ J. Hoya,${ }^{87}$ M. Hrabovsky, ${ }^{129}$ J. Hrdinka ${ }^{36}$ I. Hristova, ${ }^{19}$ J. Hrivnac, ${ }^{131}$ A. Hrynevich, ${ }^{107}$ T. Hryn'ova, ${ }^{5}$ P. J. Hsu, ${ }^{63}$ S.-C. Hsu, ${ }^{147}$ Q. Hu, ${ }^{29}$ S. Hu, ${ }^{59 c}$ Y. Huang, ${ }^{15 a}$ Z. Hubacek, ${ }^{141}$ F. Hubaut, ${ }^{100}$ M. Huebner, ${ }^{24}$ F. Huegging, ${ }^{24}$ T. B. Huffman, ${ }^{134}$ M. Huhtinen, ${ }^{36}$ R. F. H. Hunter, ${ }^{34}$ P. Huo, ${ }^{154}$ A. M. Hupe,${ }^{34}$ N. Huseynov,${ }^{7, c}$ J. Huston, ${ }^{105}$ J. Huth, ${ }^{58}$ R. Hyneman, ${ }^{104}$ G. Iacobucci, ${ }^{53}$ G. Iakovidis, ${ }^{29}$ I. Ibragimov, ${ }^{150}$ L. Iconomidou-Fayard ${ }^{131}$ Z. Idrissi,${ }^{35 e}$ P. I. Iengo, ${ }^{36}$ R. Ignazzi, ${ }^{40}$ O. Igonkina, ${ }^{119, \text { aa }}$ R. Iguchi, ${ }^{162}$ T. Iizawa ${ }^{53}$ Y. Ikegami ${ }^{80}$ M. Ikeno, ${ }^{80}$ D. Iliadis,${ }^{161}$ N. Ilic,${ }^{118}$ F. Iltzsche, ${ }^{47}$ G. Introzzi,${ }^{69 \mathrm{a}, 69 \mathrm{~b}} \mathrm{M}$. Iodice, ${ }^{73 \mathrm{a}} \mathrm{K}$. Iordanidou, ${ }^{39} \mathrm{~V}$. Ippolito, ${ }^{71 \mathrm{a}, 71 \mathrm{~b}} \mathrm{M}$. F. Isacson, ${ }^{171} \mathrm{~N}$. Ishijima, ${ }^{132} \mathrm{M}$. Ishino, ${ }^{162}$ M. Ishitsuka, ${ }^{164} \mathrm{~W}$. Islam, ${ }^{128} \mathrm{C}$. Issever, ${ }^{134} \mathrm{~S}$. Istin, ${ }^{159} \mathrm{~F}$. Ito, ${ }^{168} \mathrm{~J}$. M. Iturbe Ponce, ${ }^{62 \mathrm{a}} \mathrm{R}$. Iuppa, ${ }^{74 \mathrm{a}, 74 \mathrm{~b}} \mathrm{~A}$. Ivina, ${ }^{179}$ H. Iwasaki, ${ }^{80}$ J. M. Izen, ${ }^{43}$ V. Izzo, ${ }^{68 a}$ P. Jacka, ${ }^{140}$ P. Jackson, ${ }^{1}$ R. M. Jacobs, ${ }^{24}$ V. Jain, ${ }^{2}$ G. Jäkel, ${ }^{181}$ K. B. Jakobi, ${ }^{98}$ K. Jakobs ${ }^{51}$ S. Jakobsen, ${ }^{75}$ T. Jakoubek, ${ }^{140}$ D. O. Jamin, ${ }^{128}$ R. Jansky,${ }^{53}$ J. Janssen, ${ }^{24}$ M. Janus,${ }^{52}$ P. A. Janus, ${ }^{82 a}$ G. Jarlskog, ${ }^{95}$ N. Javadov, ${ }^{78, c}$ T. Javůrek, ${ }^{36}$ M. Javurkova, ${ }^{51}$ F. Jeanneau, ${ }^{144}$ L. Jeanty, ${ }^{18}$ J. Jejelava, ${ }^{158 a, b b}$ A. Jelinskas, ${ }^{177}$ 
P. Jenni, ${ }^{51, c c}$ J. Jeong, ${ }^{45}$ N. Jeong, ${ }^{45}$ S. Jézéquel, ${ }^{5}$ H. Ji, ${ }^{180}$ J. Jia, ${ }^{154}$ H. Jiang, ${ }^{77}$ Y. Jiang, ${ }^{59 a}$ Z. Jiang, ${ }^{152}$ S. Jiggins, ${ }^{51}$ F. A. Jimenez Morales, ${ }^{38}$ J. Jimenez Pena, ${ }^{173}$ S. Jin, ${ }^{15 c}$ A. Jinaru, ${ }^{27 b}$ O. Jinnouchi, ${ }^{164}$ H. Jivan, ${ }^{33 \mathrm{c}}$ P. Johansson, ${ }^{148}$ K. A. Johns, ${ }^{7}$ C. A. Johnson, ${ }^{64}$ W. J. Johnson, ${ }^{147}$ K. Jon-And, ${ }^{44 a, 44 b}$ R. W. L. Jones, ${ }^{88}$ S. D. Jones, ${ }^{155}$ S. Jones, ${ }^{7}$ T. J. Jones,${ }^{89}$ J. Jongmanns, ${ }^{60 a}$ P. M. Jorge, ${ }^{139 a, 139 b}$ J. Jovicevic, ${ }^{167 a}$ X. Ju, ${ }^{18}$ J. J. Junggeburth,${ }^{14}$ A. Juste Rozas, ${ }^{14, v}$ A. Kaczmarska, ${ }^{83}$ M. Kado, ${ }^{131}$ H. Kagan, ${ }^{125}$ M. Kagan, ${ }^{152}$ T. Kaji, ${ }^{178}$ E. Kajomovitz, ${ }^{159}$ C. W. Kalderon, ${ }^{95}$ A. Kaluza, ${ }^{98}$ S. Kama, ${ }^{42}$ A. Kamenshchikov, ${ }^{122}$ L. Kanjir, ${ }^{90}$ Y. Kano, ${ }^{162}$ V. A. Kantserov, ${ }^{111}$ J. Kanzaki, ${ }^{80}$ L. S. Kaplan, ${ }^{180}$ D. Kar, ${ }^{33 c}$ M. J. Kareem, ${ }^{167 b}$ E. Karentzos, ${ }^{10}$ S. N. Karpov, ${ }^{78}$ Z. M. Karpova, ${ }^{78}$ V. Kartvelishvili, ${ }^{88}$ A. N. Karyukhin, ${ }^{122}$ L. Kashif, ${ }^{180}$ R. D. Kass, ${ }^{125}$ A. Kastanas, ${ }^{44 a, 44 b}$ Y. Kataoka, ${ }^{162}$ C. Kato, ${ }^{59 d, 59 c}$ J. Katzy, ${ }^{45}$ K. Kawade, ${ }^{81}$ K. Kawagoe, ${ }^{86}$ T. Kawamoto, ${ }^{162}$ G. Kawamura, ${ }^{52}$ E. F. Kay, ${ }^{89}$ V. F. Kazanin, ${ }^{121 b, 121 a}$ R. Keeler, ${ }^{175}$ R. Kehoe, ${ }^{42}$ J. S. Keller, ${ }^{34}$ E. Kellermann, ${ }^{95}$ J. J. Kempster ${ }^{21}$ J. Kendrick, ${ }^{21}$ O. Kepka, ${ }^{140}$ S. Kersten, ${ }^{181}$ B. P. Kerševan, ${ }^{90}$ S. Ketabchi Haghighat, ${ }^{166}$ R. A. Keyes, ${ }^{102}$ M. Khader, ${ }^{172}$

F. Khalil-Zada, ${ }^{13}$ A. Khanov, ${ }^{128}$ A. G. Kharlamov, ${ }^{121 b, 121 a}$ T. Kharlamova, ${ }^{121 b, 121 a}$ E. E. Khoda, ${ }^{174}$ A. Khodinov, ${ }^{165}$

T. J. Khoo, ${ }^{53}$ E. Khramov, ${ }^{78}$ J. Khubua, ${ }^{158 b}$ S. Kido,${ }^{81}$ M. Kiehn, ${ }^{53}$ C. R. Kilby,${ }^{92}$ Y. K. Kim, ${ }^{37}$ N. Kimura,${ }^{65 a 65 c}$ O. M. Kind, ${ }^{19}$ B. T. King, ${ }^{89, a}$ D. Kirchmeier, ${ }^{47}$ J. Kirk, ${ }^{143}$ A. E. Kiryunin, ${ }^{114}$ T. Kishimoto, ${ }^{162}$ D. Kisielewska, ${ }^{82 a}$ V. Kitali, ${ }^{45}$ O. Kivernyk, ${ }^{5}$ E. Kladiva, ${ }^{28 b, a}$ T. Klapdor-Kleingrothaus, ${ }^{51}$ M. H. Klein, ${ }^{104}$ M. Klein, ${ }^{89}$ U. Klein, ${ }^{89}$ K. Kleinknecht, ${ }^{98}$ P. Klimek, ${ }^{120}$ A. Klimentov, ${ }^{29}$ T. Klingl, ${ }^{24}$ T. Klioutchnikova, ${ }^{36}$ F. F. Klitzner, ${ }^{113}$ P. Kluit, ${ }^{119}$ S. Kluth, ${ }^{114}$ E. Kneringer, ${ }^{75}$ E. B. F. G. Knoops, ${ }^{100}$ A. Knue, ${ }^{51}$ A. Kobayashi, ${ }^{162}$ D. Kobayashi, ${ }^{86}$ T. Kobayashi, ${ }^{162}$ M. Kobel, ${ }^{47}$ M. Kocian, ${ }^{152}$ P. Kodys, ${ }^{142}$ D. M. Koeck, ${ }^{113}$ P. T. Koenig, ${ }^{24}$ T. Koffas, ${ }^{34}$ E. Koffeman, ${ }^{119}$ N. M. Köhler, ${ }^{114}$ T. Koi, ${ }^{152}$ M. Kolb,${ }^{60 b}$ I. Koletsou, ${ }^{5}$ T. Kondo, ${ }^{80}$ N. Kondrashova, ${ }^{59 c}$ K. Köneke, ${ }^{51}$ A. C. König, ${ }^{118}$ T. Kono, ${ }^{124}$ R. Konoplich, ${ }^{123, d d}$ V. Konstantinides,${ }^{93}$ N. Konstantinidis,${ }^{93}$ B. Konya, ${ }^{95}$ R. Kopeliansky, ${ }^{64}$ S. Koperny, ${ }^{82 a}$ K. Korcyl, ${ }^{83}$ K. Kordas, ${ }^{161}$ G. Koren, ${ }^{160}$ A. Korn, ${ }^{93}$ I. Korolkov, ${ }^{14}$ E. V. Korolkova, ${ }^{148}$ N. Korotkova, ${ }^{112}$ O. Kortner, ${ }^{114}$ S. Kortner, ${ }^{114}$ T. Kosek, ${ }^{142}$ V. V. Kostyukhin, ${ }^{24}$ A. Kotwal, ${ }^{48}$ A. Koulouris, ${ }^{10}$ A. Kourkoumeli-Charalampidi, ${ }^{69 a, 69 b}$ C. Kourkoumelis, ${ }^{9}$ E. Kourlitis, ${ }^{148}$ V. Kouskoura, ${ }^{29}$ A. B. Kowalewska, ${ }^{83}$ R. Kowalewski, ${ }^{175}$ T. Z. Kowalski, ${ }^{82 a}$ C. Kozakai, ${ }^{162}$ W. Kozanecki, ${ }^{144}$ A. S. Kozhin, ${ }^{122}$ V. A. Kramarenko, ${ }^{112}$ G. Kramberger, ${ }^{90}$ D. Krasnopevtsev, ${ }^{59 a}$ M. W. Krasny, ${ }^{135}$ A. Krasznahorkay, ${ }^{36}$ D. Krauss, ${ }^{114}$ J. A. Kremer, ${ }^{82 a}$ J. Kretzschmar, ${ }^{89}$ P. Krieger, ${ }^{166}$ K. Krizka, ${ }^{18}$ K. Kroeninger, ${ }^{46}$ H. Kroha, ${ }^{114}$ J. Kroll, ${ }^{140}$ J. Kroll, ${ }^{136}$ J. Krstic ${ }^{16}$ U. Kruchonak, ${ }^{78}$ H. Krüger ${ }^{24}$ N. Krumnack, ${ }^{77}$ M. C. Kruse, ${ }^{48}$ T. Kubota, ${ }^{103}$ S. Kuday, ${ }^{4 b}$ J. T. Kuechler, ${ }^{181}$ S. Kuehn, ${ }^{36}$ A. Kugel, ${ }^{60 a}$ T. Kuhl, ${ }^{45}$ V. Kukhtin, ${ }^{78}$ R. Kukla, ${ }^{100}$ Y. Kulchitsky, ${ }^{106}$ S. Kuleshov, ${ }^{146 b}$ Y. P. Kulinich, ${ }^{172}$ M. Kuna,${ }^{57}$ T. Kunigo,${ }^{84}$ A. Kupco, ${ }^{140}$ T. Kupfer, ${ }^{46}$ O. Kuprash, ${ }^{160}$ H. Kurashige,${ }^{81}$ L. L. Kurchaninov, ${ }^{167 a}$ Y. A. Kurochkin, ${ }^{106}$ A. Kurova, ${ }^{111}$ M. G. Kurth, ${ }^{15 a, 15 d}$ E. S. Kuwertz, ${ }^{36}$ M. Kuze, ${ }^{164}$ J. Kvita, ${ }^{129}$ T. Kwan, ${ }^{102}$ A. La Rosa, ${ }^{114}$ J. L. La Rosa Navarro, ${ }^{79 d}$ L. La Rotonda, ${ }^{41 b, 41 a}$ F. La Ruffa, ${ }^{41 b, 41 a}$ C. Lacasta, ${ }^{173}$ F. Lacava, ${ }^{71 a, 71 b}$ J. Lacey, ${ }^{45}$ D. P. J. Lack, ${ }^{99}$ H. Lacker, ${ }^{19}$ D. Lacour, ${ }^{135}$ E. Ladygin, ${ }^{78}$ R. Lafaye ${ }^{5}$ B. Laforge, ${ }^{135}$ T. Lagouri, ${ }^{33 c}$ S. Lai, ${ }^{52}$ S. Lammers,${ }^{64}$ W. Lampl, ${ }^{7}$ E. Lançon, ${ }^{29}$ U. Landgraf, ${ }^{51}$ M. P. J. Landon, ${ }^{91}$ M. C. Lanfermann, ${ }^{53}$ V. S. Lang, ${ }^{45}$ J. C. Lange, ${ }^{52}$ R. J. Langenberg, ${ }^{36}$ A. J. Lankford, ${ }^{170}$ F. Lanni, ${ }^{29}$ K. Lantzsch,${ }^{24}$ A. Lanza, ${ }^{69 a}$ A. Lapertosa, ${ }^{54 b, 54 a}$ S. Laplace, ${ }^{135}$ J. F. Laporte, ${ }^{144}$ T. Lari, ${ }^{67 a}$ F. Lasagni Manghi, ${ }^{23 b, 23 a}$ M. Lassnig, ${ }^{36}$ T. S. Lau, ${ }^{62 a}$ A. Laudrain, ${ }^{131}$ M. Lavorgna, ${ }^{6 a, 68 b}$ M. Lazzaroni, ${ }^{67 a, 67 b}$ B. Le, ${ }^{103}$ O. Le Dortz, ${ }^{135}$ E. Le Guirriec, ${ }^{100}$ E. P. Le Quilleuc, ${ }^{144}$ M. LeBlanc, ${ }^{7}$ T. LeCompte, ${ }^{6}$ F. Ledroit-Guillon, ${ }^{57}$ C. A. Lee, ${ }^{29}$ G. R. Lee, ${ }^{146 a}$ L. Lee, ${ }^{58}$ S. C. Lee ${ }^{157}$ B. Lefebvre, ${ }^{102}$ M. Lefebvre, ${ }^{175}$ F. Legger, ${ }^{113}$ C. Leggett, ${ }^{18}$ K. Lehmann, ${ }^{151}$ N. Lehmann, ${ }^{181}$ G. Lehmann Miotto, ${ }^{36}$ W. A. Leight, ${ }^{45}$ A. Leisos, ${ }^{161, e e}$ M. A. L. Leite, ${ }^{79 d}$ R. Leitner, ${ }^{142}$ D. Lellouch, ${ }^{179, a}$ K. J. C. Leney, ${ }^{93}$ T. Lenz,${ }^{24}$ B. Lenzi ${ }^{36}$ R. Leone, ${ }^{7}$ S. Leone, ${ }^{70 a}$ C. Leonidopoulos ${ }^{49}$ G. Lerner, ${ }^{155}$ C. Leroy, ${ }^{108}$ R. Les, ${ }^{166}$ A. A. J. Lesage, ${ }^{144}$ C. G. Lester, ${ }^{32}$ M. Levchenko, ${ }^{137}$ J. Levêque, ${ }^{5}$ D. Levin, ${ }^{104}$ L. J. Levinson, ${ }^{179}$ D. Lewis,${ }^{91}$ B. Li $1{ }^{15 b}$ B. Li, ${ }^{104}$ C-Q. Li,${ }^{59 a, f f}$ H. Li, ${ }^{59 a}$ H. Li,${ }^{59 b}$ L. Li,${ }^{59 c}$ M. Li, ${ }^{15 a}$ Q. Li, ${ }^{15 a, 15 d}$ Q. Y. Li,${ }^{59 a}$ S. Li,${ }^{59 d, 59 c}$ X. Li,${ }^{59 c}$ Y. Li, ${ }^{150}$ Z. Liang, ${ }^{15 a}$ B. Liberti, ${ }^{72 \mathrm{a}}$ A. Liblong, ${ }^{166}$ K. Lie, ${ }^{62 \mathrm{c}}$ S. Liem, ${ }^{119}$ A. Limosani, ${ }^{156}$ C. Y. Lin ${ }^{32}$ K. Lin, ${ }^{105}$ T. H. Lin, ${ }^{98}$ R. A. Linck, ${ }^{64}$ J. H. Lindon, ${ }^{21}$ B. E. Lindquist, ${ }^{154}$ A. L. Lionti, ${ }^{53}$ E. Lipeles, ${ }^{136}$ A. Lipniacka, ${ }^{17}$ M. Lisovyi, ${ }^{60 b}$ T. M. Liss,${ }^{172, g g}$ A. Lister, ${ }^{174}$ A. M. Litke, ${ }^{145}$ J. D. Little, ${ }^{8}$ B. Liu, ${ }^{77}$ B. L Liu, ${ }^{6}$ H. B. Liu, ${ }^{29}$ H. Liu, ${ }^{104}$ J. B. Liu,${ }^{59 a}$ J. K. K. Liu, ${ }^{134}$ K. Liu, ${ }^{135}$ M. Liu, ${ }^{59 a}$ P. Liu, ${ }^{18}$ Y. Liu, ${ }^{15 a, 15 d}$ Y. L. Liu, ${ }^{59 a}$ Y. W. Liu, ${ }^{59 a}$ M. Livan, ${ }^{69 a, 69 b}$ A. Lleres, ${ }^{57}$ J. Llorente Merino, ${ }^{15 a}$ S. L. Lloyd,${ }^{91}$ C. Y. Lo, ${ }^{62 b}$ F. Lo Sterzo, ${ }^{42}$ E. M. Lobodzinska, ${ }^{45}$ P. Loch, ${ }^{7}$ T. Lohse, ${ }^{19}$ K. Lohwasser, ${ }^{148}$ M. Lokajicek, ${ }^{140}$ J. D. Long, ${ }^{172}$ R. E. Long, ${ }^{88}$ L. Longo ${ }^{66 a, 66 b}$ K. A. Looper, ${ }^{125}$ J. A. Lopez,${ }^{146 b}$ I. Lopez Paz, ${ }^{99}$ A. Lopez Solis, ${ }^{148}$ J. Lorenz, ${ }^{113}$ N. Lorenzo Martinez, ${ }^{5}$ M. Losada, ${ }^{22}$ P. J. Lösel, ${ }^{113}$ A. Lösle, ${ }^{51}$ X. Lou, ${ }^{45}$ X. Lou, ${ }^{15 a}$ A. Lounis, ${ }^{131}$ J. Love, ${ }^{6}$ P. A. Love, ${ }^{88}$ J. J. Lozano Bahilo, ${ }^{173}$ H. Lu, ${ }^{62 a}$ M. Lu ${ }^{59 a}$ Y. J. Lu, ${ }^{63}$ H. J. Lubatti, ${ }^{147}$ C. Luci, ${ }^{71 a, 71 b}$ A. Lucotte,${ }^{57}$ C. Luedtke, ${ }^{51}$ F. Luehring, ${ }^{64}$ I. Luise, ${ }^{135}$ L. Luminari, ${ }^{71 a}$ B. Lund-Jensen, ${ }^{153}$ M. S. Lutz, ${ }^{101}$ P. M. Luzi, ${ }^{135}$ D. Lynn, ${ }^{29}$ R. Lysak, ${ }^{140}$ E. Lytken, ${ }^{95}$ F. Lyu, ${ }^{15 a}$ V. Lyubushkin, ${ }^{78}$ T. Lyubushkina, ${ }^{78}$ H. Ma, ${ }^{29}$ L. L. Ma,${ }^{59 b}$ Y. Ma,${ }^{59 b}$ G. Maccarrone, ${ }^{50}$ A. Macchiolo, ${ }^{114}$ 
C. M. Macdonald, ${ }^{148}$ J. Machado Miguens, ${ }^{136,139 b}$ D. Madaffari, ${ }^{173}$ R. Madar, ${ }^{38}$ W. F. Mader, ${ }^{47}$ A. Madsen, ${ }^{45}$ N. Madysa, ${ }^{47}$ J. Maeda, ${ }^{81}$ K. Maekawa, ${ }^{162}$ S. Maeland, ${ }^{17}$ T. Maeno, ${ }^{29}$ M. Maerker, ${ }^{47}$ A. S. Maevskiy, ${ }^{112}$ V. Magerl, ${ }^{51}$ D. J. Mahon, ${ }^{39}$ C. Maidantchik, ${ }^{79 b}$ T. Maier, ${ }^{113}$ A. Maio, ${ }^{139 a, 139 b, 139 d}$ O. Majersky, ${ }^{28 a}$ S. Majewski, ${ }^{130}$ Y. Makida, ${ }^{80}$ N. Makovec, ${ }^{131}$ B. Malaescu, ${ }^{135}$ Pa. Malecki, ${ }^{83}$ V. P. Maleev, ${ }^{137}$ F. Malek, ${ }^{57}$ U. Mallik, ${ }^{76}$ D. Malon, ${ }^{6}$ C. Malone, ${ }^{32}$ S. Maltezos, ${ }^{10}$ S. Malyukov, ${ }^{36}$ J. Mamuzic, ${ }^{173}$ G. Mancini, ${ }^{50}$ I. Mandić, ${ }^{90}$ J. Maneira, ${ }^{139 a}$ L. Manhaes de Andrade Filho, ${ }^{79 a}$

J. Manjarres Ramos, ${ }^{47}$ K. H. Mankinen, ${ }^{95}$ A. Mann, ${ }^{113}$ A. Manousos, ${ }^{75}$ B. Mansoulie, ${ }^{144}$ J. D. Mansour, ${ }^{15 a}$ S. Manzoni, ${ }^{67 a, 67 b}$ A. Marantis, ${ }^{161}$ G. Marceca ${ }^{30}$ L. March, ${ }^{53}$ L. Marchese, ${ }^{134}$ G. Marchiori, ${ }^{135}$ M. Marcisovsky, ${ }^{140}$ C. Marcon, ${ }^{95}$ C. A. Marin Tobon, ${ }^{36}$ M. Marjanovic, ${ }^{38}$ F. Marroquim, ${ }^{79 b}$ Z. Marshall, ${ }^{18}$ M. U. F Martensson, ${ }^{171}$ S. Marti-Garcia, ${ }^{173}$ C. B. Martin, ${ }^{125}$ T. A. Martin, ${ }^{177}$ V. J. Martin, ${ }^{49}$ B. Martin dit Latour, ${ }^{17}$ M. Martinez, ${ }^{14, v}$ V. I. Martinez Outschoorn, ${ }^{101}$ S. Martin-Haugh, ${ }^{143}$ V. S. Martoiu, ${ }^{27 b}$ A. C. Martyniuk, ${ }^{93}$ A. Marzin, ${ }^{36}$ L. Masetti, ${ }^{98}$ T. Mashimo, ${ }^{162}$ R. Mashinistov, ${ }^{109}$

J. Masik, ${ }^{99}$ A. L. Maslennikov, ${ }^{121 b, 121 a}$ L. H. Mason, ${ }^{103}$ L. Massa, ${ }^{72 a, 72 b}$ P. Massarotti, ${ }^{68 a, 68 b}$ P. Mastrandrea, ${ }^{154}$

A. Mastroberardino, ${ }^{41 b, 41 a}$ T. Masubuchi, ${ }^{162}$ P. Mättig, ${ }^{181}$ J. Maurer, ${ }^{27 b}$ B. Maček, ${ }^{90}$ S. J. Maxfield, ${ }^{89}$ D. A. Maximov, ${ }^{121 b, 121 a}$ R. Mazini, ${ }^{157}$ I. Maznas, ${ }^{161}$ S. M. Mazza, ${ }^{145}$ G. Mc Goldrick, ${ }^{166}$ S. P. Mc Kee, ${ }^{104}$ T. G. McCarthy, ${ }^{114}$ L. I. McClymont, ${ }^{93}$

W. P. McCormack, ${ }^{18}$ E. F. McDonald, ${ }^{103}$ J. A. Mcfayden, ${ }^{36}$ M. A. McKay, ${ }^{42}$ K. D. McLean, ${ }^{175}$ S. J. McMahon, ${ }^{143}$ P. C. McNamara, ${ }^{103}$ C. J. McNicol, ${ }^{177}$ R. A. McPherson, ${ }^{175, m}$ J. E. Mdhluli, ${ }^{33 c}$ Z. A. Meadows, ${ }^{101}$ S. Meehan, ${ }^{147}$ T. Megy, ${ }^{51}$ S. Mehlhase, ${ }^{113}$ A. Mehta, ${ }^{89}$ T. Meideck, ${ }^{57}$ B. Meirose, ${ }^{43}$ D. Melini, ${ }^{173, \text { hh }}$ B. R. Mellado Garcia, ${ }^{33 c}$ J. D. Mellenthin, ${ }^{52}$ M. Melo, ${ }^{28 a}$ F. Meloni, ${ }^{45}$ A. Melzer, ${ }^{24}$ S. B. Menary, ${ }^{99}$ E. D. Mendes Gouveia, ${ }^{139 a}$ L. Meng, ${ }^{89}$ X. T. Meng, ${ }^{104}$ S. Menke, ${ }^{114}$ E. Meoni, ${ }^{41 b, 41 a}$ S. Mergelmeyer, ${ }^{19}$ S. A. M. Merkt, ${ }^{138}$ C. Merlassino, ${ }^{20}$ P. Mermod, ${ }^{53}$ L. Merola, ${ }^{68 a, 68 b}$ C. Meroni, ${ }^{67 a}$ F. S. Merritt, ${ }^{37}$ A. Messina, ${ }^{71 a, 71 b}$ J. Metcalfe, ${ }^{6}$ A. S. Mete, ${ }^{170}$ C. Meyer, ${ }^{136}$ J. Meyer, ${ }^{159}$ J-P. Meyer, ${ }^{144}$

H. Meyer Zu Theenhausen, ${ }^{60 a}$ F. Miano, ${ }^{155}$ R. P. Middleton, ${ }^{143}$ L. Mijović,${ }^{49}$ G. Mikenberg, ${ }^{179}$ M. Mikestikova, ${ }^{140}$ M. Mikuž, ${ }^{90}$ M. Milesi, ${ }^{103}$ A. Milic, ${ }^{166}$ D. A. Millar, ${ }^{91}$ D. W. Miller, ${ }^{37}$ A. Milov, ${ }^{179}$ D. A. Milstead, ${ }^{44 a, 44 b}$ A. A. Minaenko, ${ }^{122}$ M. Miñano Moya, ${ }^{173}$ I. A. Minashvili, ${ }^{158 b}$ A. I. Mincer, ${ }^{123}$ B. Mindur, ${ }^{82 \mathrm{a}}$ M. Mineev, ${ }^{78}$ Y. Minegishi, ${ }^{162}$ Y. Ming, ${ }^{180}$ L. M. Mir, ${ }^{14}$ A. Mirto, ${ }^{66,66 b}$ K. P. Mistry, ${ }^{136}$ T. Mitani, ${ }^{178}$ J. Mitrevski, ${ }^{113}$ V. A. Mitsou, ${ }^{173}$ M. Mittal, ${ }^{59 c}$ A. Miucci, ${ }^{20}$ P. S. Miyagawa, ${ }^{148}$ A. Mizukami, ${ }^{80}$ J. U. Mjörnmark, ${ }^{95}$ T. Mkrtchyan, ${ }^{183}$ M. Mlynarikova, ${ }^{142}$ T. Moa, ${ }^{44 a, 44 b}$ K. Mochizuki, ${ }^{108}$ P. Mogg, ${ }^{51}$ S. Mohapatra, ${ }^{39}$ S. Molander, ${ }^{44 a, 44 b}$ R. Moles-Valls, ${ }^{24}$ M. C. Mondragon, ${ }^{105}$ K. Mönig, ${ }^{45}$ J. Monk, ${ }^{40}$

E. Monnier, ${ }^{100}$ A. Montalbano, ${ }^{151}$ J. Montejo Berlingen, ${ }^{36}$ F. Monticelli, ${ }^{87}$ S. Monzani, ${ }^{67 a}$ N. Morange, ${ }^{131}$ D. Moreno, ${ }^{22}$ M. Moreno Llácer, ${ }^{36}$ P. Morettini, ${ }^{54 b}$ M. Morgenstern, ${ }^{119}$ S. Morgenstern, ${ }^{47}$ D. Mori, ${ }^{151}$ M. Morii, ${ }^{58}$ M. Morinaga, ${ }^{178}$

V. Morisbak, ${ }^{133}$ A. K. Morley, ${ }^{36}$ G. Mornacchi, ${ }^{36}$ A. P. Morris, ${ }^{93}$ J. D. Morris, ${ }^{91}$ L. Morvaj, ${ }^{154}$ P. Moschovakos, ${ }^{10}$ M. Mosidze, ${ }^{158 b}$ H. J. Moss, ${ }^{148}$ J. Moss, ${ }^{31, i i}$ K. Motohashi, ${ }^{164}$ R. Mount, ${ }^{152}$ E. Mountricha, ${ }^{36}$ E. J. W. Moyse, ${ }^{101}$ S. Muanza, ${ }^{100}$ F. Mueller, ${ }^{114}$ J. Mueller, ${ }^{138}$ R. S. P. Mueller, ${ }^{113}$ D. Muenstermann, ${ }^{88}$ G. A. Mullier, ${ }^{95}$ F. J. Munoz Sanchez, ${ }^{99}$ P. Murin, ${ }^{28 b}$ W. J. Murray, ${ }^{177,143}$ A. Murrone, ${ }^{67 a, 67 b}$ M. Muškinja, ${ }^{90}$ C. Mwewa, ${ }^{33 a}$ A. G. Myagkov, ${ }^{122, j j}$ J. Myers, $^{130}$ M. Myska, ${ }^{141}$ B. P. Nachman, ${ }^{18}$ O. Nackenhorst, ${ }^{46}$ K. Nagai, ${ }^{134}$ K. Nagano, ${ }^{80}$ Y. Nagasaka, ${ }^{61}$ M. Nagel, ${ }^{51}$ E. Nagy, ${ }^{100}$ A. M. Nairz, ${ }^{36}$ Y. Nakahama, ${ }^{116}$ K. Nakamura, ${ }^{80}$ T. Nakamura, ${ }^{162}$ I. Nakano, ${ }^{126}$ H. Nanjo, ${ }^{132}$ F. Napolitano, ${ }^{60}$ R. F. Naranjo Garcia, ${ }^{45}$ R. Narayan, ${ }^{11}$ D. I. Narrias Villar, ${ }^{60}$ I. Naryshkin, ${ }^{137}$ T. Naumann, ${ }^{45}$ G. Navarro, ${ }^{22}$ R. Nayyar, H. A. Neal, ${ }^{104, a}$ P. Y. Nechaeva, ${ }^{109}$ T. J. Neep, ${ }^{144}$ A. Negri, ${ }^{69 a, 69 b}$ M. Negrini, ${ }^{23 b}$ S. Nektarijevic, ${ }^{118}$ C. Nellist, ${ }^{52}$ M. E. Nelson, ${ }^{134}$ S. Nemecek, ${ }^{140}$ P. Nemethy, ${ }^{123}$ M. Nessi, ${ }^{36, k k}$ M. S. Neubauer, ${ }^{172}$ M. Neumann, ${ }^{181}$ P. R. Newman, ${ }^{21}$ T. Y. Ng, ${ }^{62}$ Y. S. Ng, ${ }^{19}$ Y. W. Y. Ng, ${ }^{170}$ H. D. N. Nguyen, ${ }^{100}$ T. Nguyen Manh, ${ }^{108}$ E. Nibigira, ${ }^{38}$ R. B. Nickerson, ${ }^{134}$ R. Nicolaidou, ${ }^{144}$ D. S. Nielsen, ${ }^{40}$ J. Nielsen, ${ }^{145}$ N. Nikiforou, ${ }^{11}$ V. Nikolaenko, ${ }^{122, j j}$ I. Nikolic-Audit, ${ }^{135}$ K. Nikolopoulos, ${ }^{21}$ P. Nilsson, ${ }^{29}$ Y. Ninomiya, ${ }^{80}$ A. Nisati, ${ }^{71 a}$ N. Nishu, ${ }^{59 c}$ R. Nisius, ${ }^{114}$ I. Nitsche, ${ }^{46}$ T. Nitta, ${ }^{178}$ T. Nobe, ${ }^{162}$ Y. Noguchi, ${ }^{84}$ M. Nomachi, ${ }^{132}$ I. Nomidis, ${ }^{135}$ M. A. Nomura, ${ }^{29}$ T. Nooney, ${ }^{91}$ M. Nordberg, ${ }^{36}$ N. Norjoharuddeen, ${ }^{134}$ T. Novak, ${ }^{90}$ O. Novgorodova, ${ }^{47}$ R. Novotny, ${ }^{141}$ L. Nozka, ${ }^{129}$ K. Ntekas, ${ }^{170}$ E. Nurse, ${ }^{93}$ F. Nuti, ${ }^{103}$ F. G. Oakham, ${ }^{34, d}$ H. Oberlack, ${ }^{114}$ J. Ocariz, ${ }^{135}$ A. Ochi, ${ }^{81}$ I. Ochoa, ${ }^{39}$ J. P. Ochoa-Ricoux, ${ }^{146 a}$ K. O'Connor, ${ }^{26}$ S. Oda, ${ }^{86}$ S. Odaka, ${ }^{80}$ S. Oerdek, ${ }^{52}$ A. Oh, ${ }^{99}$ S. H. Oh, ${ }^{48}$ C. C. Ohm, ${ }^{153}$ H. Oide ${ }^{54 b, 54 a}$ M. L. Ojeda, ${ }^{166}$ H. Okawa, ${ }^{168}$ Y. Okazaki, ${ }^{84}$ Y. Okumura, ${ }^{162}$ T. Okuyama, ${ }^{80}$ A. Olariu, ${ }^{27 b}$ L. F. Oleiro Seabra, ${ }^{139}$ S. A. Olivares Pino, ${ }^{146 a}$ D. Oliveira Damazio, ${ }^{29}$ J. L. Oliver, ${ }^{1}$ M. J. R. Olsson, ${ }^{37}$ A. Olszewski, ${ }^{83}$ J. Olszowska, ${ }^{83}$ D. C. O’Neil, ${ }^{151}$ A. Onofre, ${ }^{139 a, 139 e}$ K. Onogi, ${ }^{116}$ P. U. E. Onyisi, ${ }^{11}$ H. Oppen, ${ }^{133}$ M. J. Oreglia, ${ }^{37}$ G. E. Orellana, ${ }^{87}$ Y. Oren, ${ }^{160}$ D. Orestano, ${ }^{73 a, 73 b}$ N. Orlando, ${ }^{62 b}$ A. A. O'Rourke, ${ }^{45}$ R. S. Orr, ${ }^{166}$ B. Osculati, ${ }^{54 \mathrm{~b}, 54 \mathrm{a}, \mathrm{a}}$ V. O'Shea, ${ }^{56}$ R. Ospanov, ${ }^{59 \mathrm{a}}$ G. Otero y Garzon, ${ }^{30}$ H. Otono, ${ }^{86}$ M. Ouchrif, ${ }^{35 \mathrm{~d}}$ F. Ould-Saada, ${ }^{133}$ A. Ouraou, ${ }^{144}$ Q. Ouyang, ${ }^{15 a}$ M. Owen, ${ }^{56}$ R. E. Owen, ${ }^{21}$ V. E. Ozcan, ${ }^{12 \mathrm{c}}$ N. Ozturk, ${ }^{8}$ J. Pacalt, ${ }^{129}$ H. A. Pacey, ${ }^{32}$ K. Pachal, ${ }^{151}$ A. Pacheco Pages, ${ }^{14}$ L. Pacheco Rodriguez, ${ }^{144}$ C. Padilla Aranda, ${ }^{14}$ S. Pagan Griso, ${ }^{18}$ M. Paganini, ${ }^{182}$ G. Palacino, ${ }^{64}$ 
S. Palazzo, ${ }^{49}$ S. Palestini, ${ }^{36}$ M. Palka, ${ }^{82 b}$ D. Pallin, ${ }^{38}$ I. Panagoulias, ${ }^{10}$ C. E. Pandini, ${ }^{36}$ J. G. Panduro Vazquez, ${ }^{92}$ P. Pani, ${ }^{36}$ G. Panizzo, ${ }^{65 a, 65 c}$ L. Paolozzi, ${ }^{53}$ T. D. Papadopoulou, ${ }^{10}$ K. Papageorgiou, ${ }^{9, s}$ A. Paramonov, ${ }^{6}$ D. Paredes Hernandez, ${ }^{6 \mathrm{~b}}$ S. R. Paredes Saenz, ${ }^{134}$ B. Parida, ${ }^{165}$ T. H. Park, ${ }^{34}$ A. J. Parker ${ }^{88}$ K. A. Parker, ${ }^{45}$ M. A. Parker, ${ }^{32}$ F. Parodi, ${ }^{54 b, 54 a}$ J. A. Parsons ${ }^{39}$ U. Parzefall, ${ }^{51}$ V. R. Pascuzzi, ${ }^{166}$ J. M. P. Pasner, ${ }^{145}$ E. Pasqualucci, ${ }^{71 a}$ S. Passaggio, ${ }^{54 b}$ F. Pastore, ${ }^{92}$ P. Pasuwan, ${ }^{44 a, 44 b}$ S. Pataraia, ${ }^{98}$ J. R. Pater, ${ }^{99}$ A. Pathak, ${ }^{180}$ T. Pauly, ${ }^{36}$ B. Pearson, ${ }^{114}$ M. Pedersen, ${ }^{133}$ L. Pedraza Diaz, ${ }^{118}$ R. Pedro, ${ }^{139 a, 139 b}$ S. V. Peleganchuk, ${ }^{121 b, 121 a}$ O. Penc, ${ }^{140}$ C. Peng, ${ }^{15 a}$ H. Peng, ${ }^{59 a}$ B. S. Peralva, ${ }^{79 a}$ M. M. Perego, ${ }^{131}$

A. P. Pereira Peixoto, ${ }^{139 \mathrm{a}}$ D. V. Perepelitsa, ${ }^{29}$ F. Peri, ${ }^{19}$ L. Perini, ${ }^{67 a, 67 b}$ H. Pernegger, ${ }^{36}$ S. Perrella, ${ }^{68 a, 68 b}$

V. D. Peshekhonov, ${ }^{78, a}$ K. Peters, ${ }^{45}$ R. F. Y. Peters, ${ }^{99}$ B. A. Petersen, ${ }^{36}$ T. C. Petersen, ${ }^{40}$ E. Petit,${ }^{57}$ A. Petridis, ${ }^{1}$ C. Petridou, ${ }^{161}$ P. Petroff, ${ }^{131}$ M. Petrov, ${ }^{134}$ F. Petrucci, ${ }^{73 a}, 73 \mathrm{~b}$ M. Pettee, ${ }^{182}$ N. E. Pettersson, ${ }^{101}$ A. Peyaud, ${ }^{144}$ R. Pezoa, ${ }^{146 b}$ T. Pham, ${ }^{103}$ F. H. Phillips, ${ }^{105}$ P. W. Phillips, ${ }^{143}$ M. W. Phipps,${ }^{172}$ G. Piacquadio,${ }^{154}$ E. Pianori, ${ }^{18}$ A. Picazio, ${ }^{101}$ M. A. Pickering, ${ }^{134}$ R. H. Pickles, ${ }^{99}$ R. Piegaia, ${ }^{30}$ J. E. Pilcher, ${ }^{37}$ A. D. Pilkington, ${ }^{99}$ M. Pinamonti, ${ }^{72 a, 72 b}$ J. L. Pinfold, ${ }^{3}$ M. Pitt, ${ }^{179}$ L. Pizzimento, ${ }^{72 a, 72 b}$ M.-A. Pleier, ${ }^{29}$ V. Pleskot, ${ }^{142}$ E. Plotnikova, ${ }^{78}$ D. Pluth, ${ }^{77}$ P. Podberezko, ${ }^{121 b, 121 a}$ R. Poettgen,${ }^{95}$ R. Poggi ${ }^{53}$ L. Poggioli, ${ }^{131}$ I. Pogrebnyak, ${ }^{105}$ D. Pohl,${ }^{24}$ I. Pokharel,${ }^{52}$ G. Polesello, ${ }^{69 a}$ A. Poley, ${ }^{18}$ A. Policicchio, ${ }^{71 a, 71 b}$ R. Polifka, ${ }^{36}$ A. Polini, ${ }^{23 b}$ C. S. Pollard ${ }^{45}$ V. Polychronakos, ${ }^{29}$ D. Ponomarenko, ${ }^{111}$ L. Pontecorvo, ${ }^{36}$ G. A. Popeneciu, ${ }^{27 \mathrm{~d}}$ D. M. Portillo Quintero, ${ }^{135}$ S. Pospisil, ${ }^{141}$ K. Potamianos, ${ }^{45}$ I. N. Potrap,${ }^{78}$ C. J. Potter, ${ }^{32}$ H. Potti, ${ }^{11}$ T. Poulsen, ${ }^{95}$ J. Poveda, ${ }^{36}$

T. D. Powell, ${ }^{148}$ M. E. Pozo Astigarraga,${ }^{36}$ P. Pralavorio, ${ }^{100}$ S. Prell, ${ }^{77}$ D. Price, ${ }^{99}$ M. Primavera ${ }^{66 a}$ S. Prince,${ }^{102}$ N. Proklova,${ }^{111}$ K. Prokofiev, ${ }^{62 \mathrm{c}}$ F. Prokoshin, ${ }^{146 \mathrm{~b}}$ S. Protopopescu, ${ }^{29}$ J. Proudfoot, ${ }^{6}$ M. Przybycien,${ }^{82 \mathrm{a}}$ A. Puri, ${ }^{172}$ P. Puzo, ${ }^{131}$

J. Qian, ${ }^{104}$ Y. Qin, ${ }^{99}$ A. Quadt, ${ }^{52}$ M. Queitsch-Maitland, ${ }^{45}$ A. Qureshi, ${ }^{1}$ P. Rados, ${ }^{103}$ F. Ragusa, ${ }^{67 a, 67 b}$ G. Rahal, ${ }^{96}$ J. A. Raine, ${ }^{53}$ S. Rajagopalan, ${ }^{29}$ A. Ramirez Morales, ${ }^{91}$ T. Rashid, ${ }^{131}$ S. Raspopov, ${ }^{5}$ M. G. Ratti, ${ }^{67 a, 67 b}$ D. M. Rauch, ${ }^{45}$ F. Rauscher, ${ }^{113}$ S. Rave, ${ }^{98}$ B. Ravina, ${ }^{148}$ I. Ravinovich, ${ }^{179}$ J. H. Rawling, ${ }^{99}$ M. Raymond, ${ }^{36}$ A. L. Read, ${ }^{133}$ N. P. Readioff, ${ }^{57}$ M. Reale,${ }^{66 a, 66 b}$ D. M. Rebuzzi, ${ }^{69 a, 69 b}$ A. Redelbach, ${ }^{176}$ G. Redlinger,${ }^{29}$ R. Reece, ${ }^{145}$ R. G. Reed ${ }^{33 \mathrm{c}}$ K. Reeves, ${ }^{43}$ L. Rehnisch, ${ }^{19}$ J. Reichert, ${ }^{136}$ D. Reikher, ${ }^{160}$ A. Reiss,${ }^{98}$ C. Rembser ${ }^{36}$ H. Ren, ${ }^{15 a}$ M. Rescigno, ${ }^{71 a}$ S. Resconi, ${ }^{67 a}$ E. D. Resseguie, ${ }^{136}$ S. Rettie, ${ }^{174}$ E. Reynolds, ${ }^{21}$ O. L. Rezanova, ${ }^{121 b, 121 \mathrm{a}}$ P. Reznicek, ${ }^{142}$ E. Ricci, ${ }^{74 a, 74 b}$ R. Richter, ${ }^{114}$ S. Richter, ${ }^{45}$ E. Richter-Was, ${ }^{82 b}$ O. Ricken, ${ }^{24}$ M. Ridel, ${ }^{135}$ P. Rieck, ${ }^{114}$ C. J. Riegel,${ }^{181}$ O. Rifki, ${ }^{45}$ M. Rijssenbeek,${ }^{154}$ A. Rimoldi, ${ }^{69 a, 69 b}$ M. Rimoldi, ${ }^{20}$ L. Rinaldi, ${ }^{23 b}$ G. Ripellino, ${ }^{153}$ B. Ristić, ${ }^{88}$ E. Ritsch, ${ }^{36}$ I. Riu, ${ }^{14}$ J. C. Rivera Vergara, ${ }^{146 a}$ F. Rizatdinova, ${ }^{128}$ E. Rizvi, ${ }^{91}$ C. Rizzi, ${ }^{14}$ R. T. Roberts, ${ }^{99}$ S. H. Robertson, ${ }^{102, \mathrm{~m}}$ D. Robinson, ${ }^{32}$ J. E. M. Robinson, ${ }^{45}$ A. Robson, ${ }^{56}$ E. Rocco, ${ }^{98}$ C. Roda,${ }^{70 a, 70 b}$ Y. Rodina,${ }^{100}$ S. Rodriguez Bosca, ${ }^{173}$ A. Rodriguez Perez, ${ }^{14}$

D. Rodriguez Rodriguez, ${ }^{173}$ A. M. Rodríguez Vera, ${ }^{167 b}$ S. Roe,${ }^{36}$ C. S. Rogan,${ }^{58}$ O. Røhne, ${ }^{133}$ R. Röhrig, ${ }^{114}$ C. P. A. Roland, ${ }^{64}$ J. Roloff, ${ }^{58}$ A. Romaniouk ${ }^{111}$ M. Romano, ${ }^{23 b, 23 a}$ N. Rompotis, ${ }^{89}$ M. Ronzani, ${ }^{123}$ L. Roos, ${ }^{135}$ S. Rosati, ${ }^{71 a}$ K. Rosbach, ${ }^{51}$ N-A. Rosien, ${ }^{52}$ B. J. Rosser, ${ }^{136}$ E. Rossi, ${ }^{45}$ E. Rossi, ${ }^{73 a, 73 b}$ E. Rossi, ${ }^{68 a, 68 b}$ L. P. Rossi, ${ }^{54 b}$ L. Rossini, ${ }^{67 a, 67 b}$ J. H. N. Rosten, ${ }^{32}$ R. Rosten, ${ }^{14}$ M. Rotaru, ${ }^{27 b}$ J. Rothberg, ${ }_{177}$ D. Rousseau, ${ }^{131}$ D. Roy, ${ }^{33 \mathrm{c}}$ A. Rozanov, ${ }^{100}$ Y. Rozen, ${ }^{159}$

X. Ruan, ${ }^{33 \mathrm{c}}$ F. Rubbo, ${ }^{152}$ F. Rühr, ${ }^{51}$ A. Ruiz-Martinez, ${ }^{173}$ Z. Rurikova, ${ }^{51}$ N. A. Rusakovich, ${ }^{78}$ H. L. Russell, ${ }^{102}$

J. P. Rutherfoord, ${ }^{7}$ E. M. Rüttinger, ${ }^{45,11}$ Y. F. Ryabov, ${ }^{137}$ M. Rybar, ${ }^{39}$ G. Rybkin, ${ }^{131}$ S. Ryu, ${ }^{6}$ A. Ryzhov, ${ }^{12}$ G. F. Rzehorz, ${ }^{52}$

P. Sabatini, ${ }^{52}$ G. Sabato, ${ }^{119}$ S. Sacerdoti, ${ }^{131}$ H. F-W. Sadrozinski, ${ }^{145}$ R. Sadykov, ${ }^{78}$ F. Safai Tehrani, ${ }^{71 a}$ P. Saha, ${ }^{120}$ M. Sahinsoy, ${ }^{60 a}$ A. Sahu, ${ }^{181}$ M. Saimpert, ${ }^{45}$ M. Saito, ${ }^{162}$ T. Saito, ${ }^{162}$ H. Sakamoto, ${ }^{162}$ A. Sakharov, ${ }^{123, d d}$ D. Salamani, ${ }^{53}$ G. Salamanna, ${ }^{73 a, 73 b}$ J. E. Salazar Loyola, ${ }^{146 b}$ P. H. Sales De Bruin, ${ }^{171}$ D. Salihagic, ${ }^{114, a}$ A. Salnikov, ${ }^{152}$ J. Salt, ${ }^{173}$ D. Salvatore, ${ }^{41 \mathrm{~b}, 41 \mathrm{a}}$ F. Salvatore, ${ }^{155}$ A. Salvucci, ${ }^{62 \mathrm{a}, 62 \mathrm{~b}, 62 \mathrm{c}}$ A. Salzburger, ${ }^{36}$ J. Samarati, ${ }^{36}$ D. Sammel, ${ }^{51}$ D. Sampsonidis, ${ }^{161}$ D. Sampsonidou, ${ }^{161}$ J. Sánchez, ${ }^{173}$ A. Sanchez Pineda, ${ }^{65 a, 65 c}$ H. Sandaker, ${ }^{133}$ C. O. Sander, ${ }^{45}$ M. Sandhoff, ${ }^{181}$ C. Sandoval, ${ }^{22}$ D. P. C. Sankey, ${ }^{143}$ M. Sannino, ${ }^{54 b, 54 a}$ Y. Sano, ${ }^{116}$ A. Sansoni,${ }^{50}$ C. Santoni, ${ }^{38}$ H. Santos, ${ }^{139 a}$ I. Santoyo Castillo, ${ }^{155}$ A. Santra, ${ }^{173}$ A. Sapronov, ${ }^{78}$ J. G. Saraiva, ${ }^{139 a, 139 d}$ O. Sasaki, ${ }^{80}$ K. Sato, ${ }^{168}$ E. Sauvan, ${ }^{5}$ P. Savard, ${ }^{166, d}$ N. Savic, ${ }^{114}$ R. Sawada, ${ }^{162}$ C. Sawyer, ${ }^{143}$ L. Sawyer, ${ }^{94, \mathrm{~mm}}$ C. Sbarra, ${ }^{23 \mathrm{~b}}$ A. Sbrizzi, ${ }^{23 \mathrm{a}}$ T. Scanlon, ${ }^{93}$ J. Schaarschmidt, ${ }^{147}$ P. Schacht, ${ }^{114}$ B. M. Schachtner, ${ }^{113}$ D. Schaefer, ${ }^{37}$ L. Schaefer, ${ }^{136}$ J. Schaeffer,${ }^{98}$ S. Schaepe,${ }^{36}$ U. Schäfer, ${ }^{98}$ A. C. Schaffer, ${ }^{131}$ D. Schaile, ${ }^{113}$ R. D. Schamberger, ${ }^{154}$ N. Scharmberg, ${ }^{99}$ V. A. Schegelsky, ${ }^{137}$ D. Scheirich, ${ }^{142}$ F. Schenck, ${ }^{19}$ M. Schernau, ${ }^{170}$ C. Schiavi, ${ }^{54 b, 54 a}$ S. Schier, ${ }^{145}$ L. K. Schildgen, ${ }^{24}$ Z. M. Schillaci, ${ }^{26}$ E. J. Schioppa, ${ }^{36}$ M. Schioppa ${ }^{41 b, 41 a}$ K. E. Schleicher, ${ }^{51}$ S. Schlenker, ${ }^{36}$ K. R. Schmidt-Sommerfeld, ${ }^{114}$ K. Schmieden, ${ }^{36}$ C. Schmitt, ${ }^{98}$ S. Schmitt,${ }^{45}$ S. Schmitz, ${ }^{98}$

J. C. Schmoeckel, ${ }^{45}$ U. Schnoor, ${ }^{51}$ L. Schoeffel, ${ }^{144}$ A. Schoening, ${ }^{60 b}$ E. Schopf, ${ }^{134}$ M. Schott, ${ }^{98}$ J. F. P. Schouwenberg, ${ }^{118}$ J. Schovancova, ${ }^{36}$ S. Schramm, ${ }^{53}$ A. Schulte, ${ }^{98}$ H-C. Schultz-Coulon, ${ }^{60 a}$ M. Schumacher, ${ }^{51}$ B. A. Schumm, ${ }^{145}$ Ph. Schune, ${ }^{144}$ A. Schwartzman, ${ }^{152}$ T. A. Schwarz, ${ }^{104}$ Ph. Schwemling,${ }^{144}$ R. Schwienhorst, ${ }^{105}$ A. Sciandra,${ }^{24}$ G. Sciolla, ${ }^{26}$ M. Scornajenghi, ${ }^{41 \mathrm{~b}, 41 \mathrm{a}}$ F. Scuri, ${ }^{70 a}$ F. Scutti, ${ }^{103}$ L. M. Scyboz, ${ }^{114}$ C. D. Sebastiani, ${ }^{71 \mathrm{a}, 71 \mathrm{~b}}$ P. Seema, ${ }^{19}$ S. C. Seidel, ${ }^{117}$ 
A. Seiden,${ }^{145}$ T. Seiss, ${ }^{37}$ J. M. Seixas,${ }^{79 b}$ G. Sekhniaidze, ${ }^{68 a}$ K. Sekhon, ${ }^{104}$ S. J. Sekula, ${ }^{42}$ N. Semprini-Cesari, ${ }^{23 b, 23 a}$ S. Sen,${ }^{48}$ S. Senkin, ${ }^{38}$ C. Serfon, ${ }^{133}$ L. Serin,${ }^{131}$ L. Serkin, ${ }^{65 a, 65 b}$ M. Sessa,${ }^{59 a}$ H. Severini, ${ }^{127}$ F. Sforza, ${ }^{169}$ A. Sfyrla, ${ }^{53}$ E. Shabalina, ${ }^{52}$ J. D. Shahinian, ${ }^{145}$ N. W. Shaikh, ${ }^{44 a, 44 b}$ D. Shaked Renous, ${ }^{179}$ L. Y. Shan, ${ }^{15 a}$ R. Shang, ${ }^{172}$ J. T. Shank, ${ }^{25}$ M. Shapiro, ${ }^{18}$ A. Sharma, ${ }^{134}$ A. S. Sharma, ${ }^{1}$ P. B. Shatalov, ${ }^{110}$ K. Shaw, ${ }^{155}$ S. M. Shaw, ${ }^{99}$ A. Shcherbakova, ${ }^{137}$ Y. Shen, ${ }^{127}$ N. Sherafati ${ }^{34}$ A. D. Sherman ${ }^{25}$ P. Sherwood, ${ }^{93}$ L. Shi, ${ }^{157, \text { nn }}$ S. Shimizu, ${ }^{80}$ C. O. Shimmin, ${ }^{182}$ Y. Shimogama, ${ }^{178}$ M. Shimojima, ${ }^{115}$ I. P. J. Shipsey, ${ }^{134}$ S. Shirabe, ${ }^{86}$ M. Shiyakova, ${ }^{78,00}$ J. Shlomi, ${ }^{179}$ A. Shmeleva, ${ }^{109}$ D. Shoaleh Saadi, ${ }^{108}$ M. J. Shochet, ${ }^{37}$ S. Shojaii, ${ }^{103}$ D. R. Shope, ${ }^{127}$ S. Shrestha, ${ }^{125}$ E. Shulga, ${ }^{111}$ P. Sicho, ${ }^{140}$ A. M. Sickles, ${ }^{172}$ P. E. Sidebo, ${ }^{153}$ E. Sideras Haddad, ${ }^{33 c}$ O. Sidiropoulou, ${ }^{36}$ A. Sidoti, ${ }^{23 b, 23 a}$ F. Siegert, ${ }^{47}$ Dj. Sijacki, ${ }^{16}$ J. Silva, ${ }^{139 a}$ M. Silva Jr., ${ }^{180}$ M. V. Silva Oliveira, ${ }^{79 a}$ S. B. Silverstein, ${ }^{44 a}$ S. Simion, ${ }^{131}$ E. Simioni, ${ }^{98}$ M. Simon,,${ }^{98}$ R. Simoniello, ${ }^{98}$ P. Sinervo, ${ }^{166}$ N. B. Sinev, ${ }^{130}$ M. Sioli, ${ }^{23 b, 23 a}$ I. Siral,${ }^{104}$ S. Yu. Sivoklokov, ${ }^{112}$ J. Sjölin, ${ }^{44 a, 44 b}$ P. Skubic, ${ }^{127}$ M. Slater,${ }^{21}$ T. Slavicek, ${ }^{141}$ M. Slawinska, ${ }^{83}$ K. Sliwa, ${ }^{169}$ R. Slovak, ${ }^{142}$ V. Smakhtin, ${ }^{179}$ B. H. Smart, ${ }^{5}$ J. Smiesko, ${ }^{28 a}$ N. Smirnov, ${ }^{111}$ S. Yu. Smirnov, ${ }^{111}$ Y. Smirnov, ${ }^{111}$ L. N. Smirnova, ${ }^{112, p p}$ O. Smirnova, ${ }^{95}$ J. W. Smith, ${ }^{52}$ M. Smizanska ${ }^{88}$ K. Smolek, ${ }^{141}$ A. Smykiewicz, ${ }^{83}$ A. A. Snesarev, ${ }^{109}$ I. M. Snyder, ${ }^{130}$ S. Snyder, ${ }^{29}$ R. Sobie, ${ }^{175, m}$ A. M. Soffa, ${ }^{170}$ A. Soffer, ${ }^{160}$ A. Søgaard, ${ }^{49}$ D. A. Soh, ${ }^{157}$ G. Sokhrannyi, ${ }^{90}$ C. A. Solans Sanchez, ${ }^{36}$ M. Solar, ${ }^{141}$ E. Yu. Soldatov, ${ }^{111}$ U. Soldevila, ${ }^{173}$ A. A. Solodkov, ${ }^{122}$ A. Soloshenko, ${ }^{78}$ O. V. Solovyanov, ${ }^{122}$ V. Solovyev, ${ }^{137}$ P. Sommer, ${ }^{148}$ H. Son, ${ }^{169}$ W. Song, ${ }^{143}$ W. Y. Song, ${ }^{167 b}$ A. Sopczak, ${ }^{141}$ F. Sopkova, ${ }^{28 b}$ C. L. Sotiropoulou, ${ }^{70 a, 70 b}$ S. Sottocornola, ${ }^{69 a, 69 b}$ R. Soualah, ${ }^{65 a, 65 c, 4 q}$ A. M. Soukharev, ${ }^{121 b, 121 a}$ D. South, ${ }^{45}$ B. C. Sowden, ${ }^{92}$ S. Spagnolo, ${ }^{66 a, 66 b}$ M. Spalla, ${ }^{114}$ M. Spangenberg, ${ }^{177}$ F. Spanò,${ }^{92}$ D. Sperlich, ${ }^{19}$ T. M. Spieker, ${ }^{60 a}$ R. Spighi, ${ }^{23 b}$ G. Spigo, ${ }^{36}$ L. A. Spiller, ${ }^{103}$ D. P. Spiteri, ${ }^{56}$ M. Spousta, ${ }^{142}$ A. Stabile,${ }^{67 a, 67 b}$ R. Stamen, ${ }^{60 a}$ S. Stamm, ${ }^{19}$ E. Stanecka, ${ }^{83}$ R. W. Stanek, ${ }^{6}$ C. Stanescu, ${ }^{73 a}$ B. Stanislaus, ${ }^{134}$ M. M. Stanitzki, ${ }^{45}$ B. Stapf, ${ }^{119}$ S. Stapnes, ${ }^{133}$ E. A. Starchenko, ${ }^{122}$ G. H. Stark, ${ }^{37}$ J. Stark, ${ }^{57}$ S. H Stark, ${ }^{40}$ P. Staroba, ${ }^{140}$ P. Starovoitov, ${ }^{60}$ S. Stärz, ${ }^{36}$ R. Staszewski, ${ }^{83}$ M. Stegler, ${ }^{45}$ P. Steinberg, ${ }^{29}$ B. Stelzer, ${ }^{151}$ H. J. Stelzer, ${ }^{36}$ O. Stelzer-Chilton, ${ }^{167 a}$ H. Stenzel,,${ }^{55}$ T. J. Stevenson, ${ }^{155}$ G. A. Stewart, ${ }^{36}$ M. C. Stockton, ${ }^{36}$ G. Stoicea, ${ }^{27 b}$ P. Stolte, ${ }^{52}$ S. Stonjek, ${ }^{114}$ A. Straessner, ${ }^{47}$ J. Strandberg, ${ }^{153}$ S. Strandberg, ${ }^{44 a, 44 b}$ M. Strauss, ${ }^{127}$ P. Strizenec, ${ }^{28 b}$ R. Ströhmer, ${ }^{176}$ D. M. Strom, ${ }^{130}$ R. Stroynowski, ${ }^{42}$ A. Strubig, ${ }^{49}$ S. A. Stucci, ${ }^{29}$ B. Stugu, ${ }^{17}$ J. Stupak, ${ }^{127}$ N. A. Styles, ${ }^{45}$ D. Su, ${ }^{152}$ J. Su, ${ }^{138}$ S. Suchek, ${ }^{60 a}$ Y. Sugaya ${ }^{132}$ M. Suk, ${ }^{141}$ V. V. Sulin, ${ }^{109}$ M. J. Sullivan ${ }^{89}$ D. M. S. Sultan, ${ }^{53}$ S. Sultansoy, ${ }^{4 c}$ T. Sumida,${ }^{84}$ S. Sun, ${ }^{104}$ X. Sun, ${ }^{3}$ K. Suruliz, ${ }^{155}$ C. J. E. Suster, ${ }^{156}$ M. R. Sutton, ${ }^{155}$ S. Suzuki, ${ }^{80}$ M. Svatos, ${ }^{140}$ M. Swiatlowski, ${ }^{37}$ S. P. Swift, ${ }^{2}$ A. Sydorenko, ${ }^{98}$ I. Sykora, ${ }^{28 a}$ T. Sykora, ${ }^{142}$ D. Ta, ${ }^{98}$ K. Tackmann, ${ }^{45, \text { rr }}$ J. Taenzer, ${ }^{160}$ A. Taffard, ${ }^{170}$ R. Tafirout, ${ }^{167 a}$ E. Tahirovic, ${ }^{91}$ N. Taiblum, ${ }^{160}$ H. Takai ${ }^{29}$ R. Takashima ${ }^{85}$ E. H. Takasugi,${ }^{114}$ K. Takeda ${ }^{81}$ T. Takeshita, ${ }^{149}$ Y. Takubo, ${ }^{80}$ M. Talby,${ }^{100}$ A. A. Talyshev, ${ }^{121 b, 121 \mathrm{a}}$ J. Tanaka ${ }^{162}$ M. Tanaka,${ }^{164}$ R. Tanaka ${ }^{131}$ B. B. Tannenwald,${ }^{125}$ S. Tapia Araya, ${ }^{146 b}$ S. Tapprogge, ${ }^{98}$ A. Tarek Abouelfadl Mohamed, ${ }^{135}$ S. Tarem, ${ }^{159}$ G. Tarna, ${ }^{27 b, p}$ G. F. Tartarelli, ${ }^{67 a}$ P. Tas, ${ }^{142}$ M. Tasevsky, ${ }^{140}$ T. Tashiro, ${ }^{84}$ E. Tassi, ${ }^{41 \mathrm{~b}, 41 \mathrm{a}}$ A. Tavares Delgado, ${ }^{139 a, 139 \mathrm{~b}}$ Y. Tayalati, ${ }^{35 \mathrm{e}}$ A. C. Taylor, ${ }^{117}$ A. J. Taylor, ${ }^{49}$ G. N. Taylor, ${ }^{103}$ P. T. E. Taylor, ${ }^{103}$ W. Taylor, ${ }^{167 b}$ A. S. Tee, ${ }^{88}$ P. Teixeira-Dias, ${ }^{92}$ H. Ten Kate, ${ }^{36}$ J. J. Teoh,${ }^{119}$ S. Terada ${ }^{80}$ K. Terashi,${ }^{162}$ J. Terron, ${ }^{97}$ S. Terzo, ${ }^{14}$ M. Testa,${ }^{50}$ R. J. Teuscher, ${ }^{166, \mathrm{~m}}$ S. J. Thais, ${ }^{182}$ T. Theveneaux-Pelzer, ${ }^{45}$ F. Thiele, ${ }^{40}$ D. W. Thomas, ${ }^{92}$ J. P. Thomas, ${ }^{21}$

A. S. Thompson, ${ }^{56}$ P. D. Thompson, ${ }^{21}$ L. A. Thomsen, ${ }^{182}$ E. Thomson, ${ }^{136}$ Y. Tian, ${ }^{39}$ R. E. Ticse Torres, ${ }^{52}$

V. O. Tikhomirov, ${ }^{109, s s}$ Yu. A. Tikhonov, ${ }^{121 b, 121 a}$ S. Timoshenko, ${ }^{111}$ P. Tipton, ${ }^{182}$ S. Tisserant,${ }^{100}$ K. Todome,${ }^{164}$ S. Todorova-Nova, ${ }^{5}$ S. Todt,${ }^{47}$ J. Tojo, ${ }^{86}$ S. Tokár, ${ }^{28 a}$ K. Tokushuku, ${ }^{80}$ E. Tolley, ${ }^{125}$ K. G. Tomiwa, ${ }^{33 c}$ M. Tomoto, ${ }^{116}$ L. Tompkins, ${ }^{152} \mathrm{~K}$. Toms,${ }^{117}$ B. Tong,${ }^{58}$ P. Tornambe,${ }^{51}$ E. Torrence, ${ }^{130}$ H. Torres,${ }^{47}$ E. Torró Pastor, ${ }^{147}$ C. Tosciri,,${ }^{134}$ J. Toth, ${ }^{100, t \mathrm{tt}}$ F. Touchard, ${ }^{100}$ D. R. Tovey, ${ }^{148}$ C. J. Treado, ${ }^{123}$ T. Trefzger, ${ }^{176}$ F. Tresoldi, ${ }^{155}$ A. Tricoli, ${ }^{29}$ I. M. Trigger, ${ }^{167 a}$ S. Trincaz-Duvoid, ${ }^{135}$ M. F. Tripiana, ${ }^{14}$ W. Trischuk, ${ }^{166}$ B. Trocmé,${ }^{57}$ A. Trofymov, ${ }^{131}$ C. Troncon, ${ }^{67 a}$ M. Trovatelli, ${ }^{175}$ F. Trovato, ${ }^{155}$ L. Truong ${ }^{33 b}$ M. Trzebinski, ${ }^{83}$ A. Trzupek ${ }^{83}$ F. Tsai,${ }^{45}$ J. C-L. Tseng, ${ }^{134}$ P. V. Tsiareshka, ${ }^{106}$ A. Tsirigotis, ${ }^{161}$ N. Tsirintanis, ${ }^{9}$ V. Tsiskaridze, ${ }^{154}$ E. G. Tskhadadze, ${ }^{158 a}$ I. I. Tsukerman, ${ }^{110}$ V. Tsulaia, ${ }^{18}$ S. Tsuno, ${ }^{80}$ D. Tsybychev, ${ }^{154}$ Y. Tu, ${ }^{62 \mathrm{~b}}$ A. Tudorache, ${ }^{27 \mathrm{~b}}$ V. Tudorache, ${ }^{27 \mathrm{~b}}$ T. T. Tulbure, ${ }^{27 \mathrm{a}}$ A. N. Tuna, ${ }^{58}$ S. Turchikhin, ${ }^{78}$ D. Turgeman, ${ }^{179}$ I. Turk Cakir, ${ }^{4 b, u u}$ R. J. Turner, ${ }^{21}$ R. T. Turra, ${ }^{67 a}$ P. M. Tuts, ${ }^{39}$ E. Tzovara, ${ }^{98}$ G. Ucchielli, ${ }^{46}$ I. Ueda, ${ }^{80}$ M. Ughetto, ${ }^{44 a, 44 b}$ F. Ukegawa, ${ }^{168}$ G. Unal, ${ }^{36}$ A. Undrus, ${ }^{29}$ G. Unel, ${ }^{170}$ F. C. Ungaro, ${ }^{103}$ Y. Unno,${ }^{80}$ K. Uno, ${ }^{162}$ J. Urban,${ }^{28 b}$ P. Urquijo, ${ }^{103}$ P. Urrejola,${ }^{98}$ G. Usai, ${ }^{8}$ J. Usui, ${ }^{80}$ L. Vacavant, ${ }^{100}$ V. Vacek,${ }^{141}$ B. Vachon,${ }^{102}$ K. O. H. Vadla, ${ }^{133}$ A. Vaidya, ${ }^{93}$ C. Valderanis, ${ }^{113}$ E. Valdes Santurio, ${ }^{44 a, 44 b}$ M. Valente ${ }^{53}$ S. Valentinetti, ${ }^{23 b, 23 a}$ A. Valero, ${ }^{173}$ L. Valéry, ${ }^{45}$ R. A. Vallance, ${ }^{21}$ A. Vallier, ${ }^{5}$ J. A. Valls Ferrer, ${ }^{173}$ T. R. Van Daalen, ${ }^{14}$ H. Van der Graaf, ${ }^{119}$ P. Van Gemmeren, ${ }^{6}$ I. Van Vulpen, ${ }^{119}$ M. Vanadia, ${ }^{72 a, 72 b}$ W. Vandelli, ${ }^{36}$ A. Vaniachine, ${ }^{165}$ P. Vankov,${ }^{119}$ R. Vari, ${ }^{71 a}$ E. W. Varnes, ${ }^{7}$ C. Varni,${ }^{54 b, 54 a}$ T. Varol, ${ }^{42}$ D. Varouchas, ${ }^{131}$ K. E. Varvell, ${ }^{156}$ G. A. Vasquez, ${ }^{146 b}$ J. G. Vasquez, ${ }^{182}$ F. Vazeille, ${ }^{38}$ D. Vazquez Furelos, ${ }^{14}$ T. Vazquez Schroeder, ${ }^{36}$ 
J. Veatch, ${ }^{52}$ V. Vecchio, ${ }^{73 a, 73 b}$ L. M. Veloce, ${ }^{166}$ F. Veloso, ${ }^{139 a, 139 c}$ S. Veneziano, ${ }^{71 a}$ A. Ventura, ${ }^{66 a, 66 b}$ N. Venturi, ${ }^{36}$ V. Vercesi, ${ }^{69 \mathrm{a}}$ M. Verducci, ${ }^{73 a, 73 \mathrm{~b}}$ C. M. Vergel Infante, ${ }^{77}$ C. Vergis, ${ }^{24}$ W. Verkerke, ${ }^{119}$ A. T. Vermeulen, ${ }^{119}$ J. C. Vermeulen, ${ }^{119}$ M. C. Vetterli, ${ }^{151, \mathrm{~d}}$ N. Viaux Maira, ${ }^{146 \mathrm{~b}}$ M. Vicente Barreto Pinto, ${ }^{53}$ I. Vichou, ${ }^{172, \mathrm{a}}$ T. Vickey, ${ }^{148}$ O. E. Vickey Boeriu, ${ }^{148}$ G. H. A. Viehhauser, ${ }^{134}$ S. Viel, ${ }^{18}$ L. Vigani, ${ }^{134}$ M. Villa, ${ }^{23 b, 23 a}$ M. Villaplana Perez, ${ }^{67,67 b}$ E. Vilucchi, ${ }^{50}$ M. G. Vincter, ${ }^{34}$ V. B. Vinogradov, ${ }^{78}$ A. Vishwakarma, ${ }^{45}$ C. Vittori, ${ }^{23 b, 23 a}$ I. Vivarelli, ${ }^{155}$ S. Vlachos, ${ }^{10}$ M. Vogel ${ }^{181}$ P. Vokac, ${ }^{141}$ G. Volpi,${ }^{14}$ S. E. von Buddenbrock, ${ }^{33 c}$ E. Von Toerne, ${ }^{24}$ V. Vorobel,,${ }^{142}$ K. Vorobev, ${ }^{111}$ M. Vos, ${ }^{173}$ J. H. Vossebeld ${ }^{89}$ N. Vranjes,${ }^{16}$ M. Vranjes Milosavljevic, ${ }^{16}$ V. Vrba, ${ }^{141}$ M. Vreeswijk ${ }^{119}$ T. Šfiligoj ${ }^{90}$ R. Vuillermet,${ }^{36}$ I. Vukotic, ${ }^{37}$ T. Ženiš, ${ }^{28 a}$ L. Živković, ${ }^{16}$ P. Wagner, ${ }^{24}$ W. Wagner, ${ }^{181}$ J. Wagner-Kuhr, ${ }^{113}$ H. Wahlberg, ${ }^{87}$ S. Wahrmund, ${ }^{47}$ K. Wakamiya, ${ }^{81}$ V. M. Walbrecht, ${ }^{114}$ J. Walder, ${ }^{88}$ R. Walker, ${ }^{13}$ S. D. Walker, ${ }^{92}$ W. Walkowiak, ${ }^{150}$ V. Wallangen, ${ }^{44 a, 44 b}$ A. M. Wang, ${ }^{58}$ C. Wang, ${ }^{59 b, p}$ F. Wang, ${ }^{180}$ H. Wang, ${ }^{18}$ H. Wang, ${ }^{3}$ J. Wang, ${ }^{156}$ J. Wang, ${ }^{60 b}$ P. Wang, ${ }^{42}$ Q. Wang, ${ }^{127}$ R.-J. Wang, ${ }^{135}$ R. Wang, ${ }^{59 a}$ R. Wang, ${ }^{6}$ S. M. Wang, ${ }^{157}$ W. T. Wang, ${ }^{59 a}$ W. Wang, ${ }^{15 c, v v}$ W. X. Wang, ${ }^{59 a, v v}$ Y. Wang, ${ }^{59 a, f f}$ Z. Wang, ${ }^{59 \mathrm{c}}$ C. Wanotayaroj, ${ }^{45}$ A. Warburton, ${ }^{102}$ C. P. Ward ${ }^{32}$ D. R. Wardrope, ${ }^{93}$ A. Washbrook, ${ }^{49}$ P. M. Watkins, ${ }^{21}$ A. T. Watson, ${ }^{21}$ M. F. Watson, ${ }^{21}$ G. Watts, ${ }^{147}$ S. Watts,${ }^{99}$ B. M. Waugh, ${ }^{93}$ A. F. Webb,${ }^{11}$ S. Webb, ${ }^{98}$ C. Weber, ${ }^{182}$ M. S. Weber, ${ }^{20}$ S. A. Weber, ${ }^{34}$ S. M. Weber, ${ }^{60 a}$ A. R. Weidberg, ${ }^{134}$ J. Weingarten, ${ }^{46}$ M. Weirich, ${ }^{98}$ C. Weiser, ${ }^{51}$ P. S. Wells,${ }^{36}$ T. Wenaus ${ }^{29}$ T. Wengler, ${ }^{36}$ S. Wenig, ${ }^{36}$ N. Wermes, ${ }^{24}$ M. D. Werner,${ }^{77}$ P. Werner, ${ }^{36}$ M. Wessels, ${ }^{60 a}$ T. D. Weston, ${ }^{20}$ K. Whalen, ${ }^{130}$ N. L. Whallon, ${ }^{147}$ A. M. Wharton, ${ }^{88}$ A. S. White, ${ }^{104}$ A. White,${ }^{8}$ M. J. White, ${ }^{1}$ R. White, ${ }^{146 b}$ D. Whiteson, ${ }^{170}$ B. W. Whitmore, ${ }^{88}$ F. J. Wickens, ${ }^{143}$ W. Wiedenmann, ${ }^{180}$ M. Wielers, ${ }^{143}$ C. Wiglesworth, ${ }^{40}$ L. A. M. Wiik-Fuchs, ${ }^{51}$ F. Wilk, ${ }^{99}$ H. G. Wilkens, ${ }^{36}$ L. J. Wilkins, ${ }^{92}$ H. H. Williams, ${ }^{136}$ S. Williams, ${ }^{32}$ C. Willis, ${ }^{105}$ S. Willocq, ${ }^{101}$ J. A. Wilson, ${ }^{21}$ I. Wingerter-Seez,${ }^{5}$ E. Winkels, ${ }^{155}$ F. Winklmeier, ${ }^{130}$ O. J. Winston, ${ }^{155}$ B. T. Winter, ${ }^{51}$ M. Wittgen,${ }^{152}$ M. Wobisch, ${ }^{94}$ A. Wolf, ${ }^{98}$ T. M. H. Wolf, ${ }^{119}$ R. Wolff, ${ }^{100}$ M. W. Wolter, ${ }^{83}$ H. Wolters, ${ }^{139 a, 139 c}$ V. W. S. Wong, ${ }^{174}$ N. L. Woods, ${ }^{145}$ S. D. Worm, ${ }^{21}$ B. K. Wosiek, ${ }^{83}$ K. W. Woźniak, ${ }^{83}$ K. Wraight, ${ }^{56}$ M. Wu, ${ }^{37}$ S. L. Wu ${ }^{180}$ X. Wu, ${ }^{53}$ Y. Wu, ${ }^{59 a}$ T. R. Wyatt, ${ }^{99}$ B. M. Wynne, ${ }^{49}$ S. Xella, ${ }^{40}$ Z. Xi ${ }^{104}$ L. Xia, ${ }^{177}$ D. Xu, ${ }^{15 a}$ H. Xu, ${ }^{59 a, p}$ L. Xu, ${ }^{29}$ T. Xu, ${ }^{144}$ W. Xu, ${ }^{104}$ B. Yabsley, ${ }^{156}$ S. Yacoob, ${ }^{33 a}$ K. Yajima, ${ }^{132}$ D. P. Yallup, ${ }^{93}$ D. Yamaguchi, ${ }^{164}$ Y. Yamaguchi, ${ }^{164}$ A. Yamamoto, ${ }^{80}$ T. Yamanaka, ${ }^{162}$ F. Yamane, ${ }^{81}$ M. Yamatani, ${ }^{162}$ T. Yamazaki, ${ }^{162}$ Y. Yamazaki, ${ }^{81}$ Z. Yan, ${ }^{25}$ H. J. Yang, ${ }^{59 c, 59 d}$ H. T. Yang, ${ }^{18}$ S. Yang,${ }^{76}$ Y. Yang, ${ }^{162}$ Z. Yang, ${ }^{17}$ W-M. Yao, ${ }^{18}$ Y. C. Yap, ${ }^{45}$ Y. Yasu, ${ }^{80}$ E. Yatsenko, ${ }^{59 c, 59 d}$ J. Ye, ${ }^{42}$ S. Ye, ${ }^{29}$ I. Yeletskikh, ${ }^{78}$ E. Yigitbasi, ${ }^{25}$ E. Yildirim, ${ }^{98}$ K. Yorita, ${ }^{178}$ K. Yoshihara, ${ }^{136}$ C. J. S. Young, ${ }^{36}$ C. Young, ${ }^{152}$ J. Yu, ${ }^{77}$ J. Yu, ${ }^{8}$ X. Yue, ${ }^{60 a}$ S. P. Y. Yuen, ${ }^{24}$ B. Zabinski ${ }^{83}$ G. Zacharis, ${ }^{10}$ E. Zaffaroni, ${ }^{53}$ R. Zaidan, ${ }^{14}$ A. M. Zaitsev, ${ }^{122, j j}$ T. Zakareishvili, ${ }^{158 b}$ N. Zakharchuk, ${ }^{34}$ J. Zalieckas, ${ }^{17}$ S. Zambito, ${ }^{58}$ D. Zanzi ${ }^{36}$ D. R. Zaripovas, ${ }^{56}$ S. V. Zeißner, ${ }^{46}$ C. Zeitnitz, ${ }^{181}$ G. Zemaityte, ${ }^{134}$ J. C. Zeng, ${ }^{172}$ Q. Zeng, ${ }^{152}$ O. Zenin, ${ }^{122}$ D. Zerwas, ${ }^{131}$ M. Zgubič, ${ }^{134}$ D. F. Zhang, ${ }^{59 b}$ D. Zhang, ${ }^{104}$ F. Zhang, ${ }^{180}$ G. Zhang, ${ }^{59 a}$ G. Zhang, ${ }^{15 b}$ H. Zhang, ${ }^{15 c}$ J. Zhang, ${ }^{6}$ L. Zhang, ${ }^{15 \mathrm{c}}$ L. Zhang, ${ }^{59 \mathrm{a}}$ M. Zhang, ${ }^{172}$ P. Zhang, ${ }^{15 \mathrm{c}}$ R. Zhang, ${ }^{59 \mathrm{a}}$ R. Zhang, ${ }^{24}$ X. Zhang, ${ }^{59 \mathrm{~b}}$ Y. Zhang, ${ }^{15,15 \mathrm{~d}}$ Z. Zhang, ${ }^{131}$ P. Zhao, ${ }^{48}$ Y. Zhao, ${ }^{596,131, x}$ Z. Zhao, ${ }^{59 a}$ A. Zhemchugov ${ }^{78}$ Z. Zheng, ${ }^{104}$ D. Zhong, ${ }^{172}$ B. Zhou, ${ }^{104}$ C. Zhou, ${ }^{180}$ L. Zhou, ${ }^{42}$ M. S. Zhou, ${ }^{15 a, 15 d}$ M. Zhou, ${ }^{154}$ N. Zhou, ${ }^{59 c}$ Y. Zhou, ${ }^{7}$ C. G. Zhu, ${ }^{59 b}$ H. L. Zhu, ${ }^{59 a}$ H. Zhu, ${ }^{15 a}$ J. Zhu, ${ }^{104}$ Y. Zhu, ${ }^{59 a}$ X. Zhuang, ${ }^{15 a}$ K. Zhukov, ${ }^{109}$ V. Zhulanov, ${ }^{121 b, 121 a}$ A. Zibell, ${ }^{176}$ D. Zieminska, ${ }^{64}$ N. I. Zimine, ${ }^{78}$ S. Zimmermann, ${ }^{51}$ Z. Zinonos, ${ }^{114}$ M. Ziolkowski, ${ }^{150}$ G. Zobernig, ${ }^{180}$ A. Zoccoli, ${ }^{23 b, 23 a}$ K. Zoch, ${ }^{52}$ T. G. Zorbas, ${ }^{148}$ R. Zou, ${ }^{37}$ M. Zur Nedden, ${ }^{19}$ and L. Zwalinski ${ }^{36}$

(ATLAS Collaboration)

${ }^{1}$ Department of Physics, University of Adelaide, Adelaide, Australia

${ }^{2}$ Physics Department, SUNY Albany, Albany, New York, USA

${ }^{3}$ Department of Physics, University of Alberta, Edmonton, Alberta, Canada

${ }^{4 a}$ Department of Physics, Ankara University, Ankara, Turkey

${ }^{4 \mathrm{~b}}$ Istanbul Aydin University, Istanbul, Turkey

${ }^{4 \mathrm{c}}$ Division of Physics, TOBB University of Economics and Technology, Ankara, Turkey

${ }^{5}$ LAPP, Université Grenoble Alpes, Université Savoie Mont Blanc, CNRS/IN2P3, Annecy, France

${ }^{6}$ High Energy Physics Division, Argonne National Laboratory, Argonne, Illinois, USA

${ }^{7}$ Department of Physics, University of Arizona, Tucson, Arizona, USA

${ }^{8}$ Department of Physics, University of Texas at Arlington, Arlington, Texas, USA

${ }^{9}$ Physics Department, National and Kapodistrian University of Athens, Athens, Greece

${ }^{10}$ Physics Department, National Technical University of Athens, Zografou, Greece

${ }^{11}$ Department of Physics, University of Texas at Austin, Austin, Texas, USA

${ }^{12 a}$ Bahcesehir University, Faculty of Engineering and Natural Sciences, Istanbul, Turkey 
${ }^{12 \mathrm{~b}}$ Istanbul Bilgi University, Faculty of Engineering and Natural Sciences, Istanbul, Turkey

${ }^{12 \mathrm{c}}$ Department of Physics, Bogazici University, Istanbul, Turkey

${ }^{12 \mathrm{~d} D e p a r t m e n t}$ of Physics Engineering, Gaziantep University, Gaziantep, Turkey

${ }^{13}$ Institute of Physics, Azerbaijan Academy of Sciences, Baku, Azerbaijan

${ }^{14}$ Institut de Física d'Altes Energies (IFAE), Barcelona Institute of Science and Technology,

Barcelona, Spain

${ }^{15 a}$ Institute of High Energy Physics, Chinese Academy of Sciences, Beijing, China

${ }^{15 \mathrm{~b}}$ Physics Department, Tsinghua University, Beijing, China

${ }^{15 \mathrm{c}}$ Department of Physics, Nanjing University, Nanjing, China

${ }^{15 \mathrm{~d}}$ University of Chinese Academy of Science (UCAS), Beijing, China

${ }^{16}$ Institute of Physics, University of Belgrade, Belgrade, Serbia

${ }^{17}$ Department for Physics and Technology, University of Bergen, Bergen, Norway

${ }^{18}$ Physics Division, Lawrence Berkeley National Laboratory and University of California, Berkeley, California, USA

${ }^{19}$ Institut für Physik, Humboldt Universität zu Berlin, Berlin, Germany

${ }^{20}$ Albert Einstein Center for Fundamental Physics and Laboratory for High Energy Physics, University of Bern, Bern, Switzerland

${ }^{21}$ School of Physics and Astronomy,

University of Birmingham, Birmingham, United Kingdom

${ }^{22}$ Facultad de Ciencias y Centro de Investigaciónes, Universidad Antonio Nariño, Bogota, Colombia

${ }^{23 a}$ INFN Bologna and Universita' di Bologna, Dipartimento di Fisica, Italy

${ }^{23 \mathrm{~b}}$ INFN Sezione di Bologna, Bologna, Italy

${ }^{24}$ Physikalisches Institut, Universität Bonn, Bonn, Germany

${ }^{25}$ Department of Physics, Boston University, Boston, Massachusetts, USA

${ }^{26}$ Department of Physics, Brandeis University, Waltham, Massachusetts, USA

${ }^{27}$ Transilvania University of Brasov, Brasov, Romania

${ }^{27 \mathrm{~b}}$ Horia Hulubei National Institute of Physics and Nuclear Engineering, Bucharest, Romania

${ }^{27 \mathrm{c}}$ Department of Physics, Alexandru Ioan Cuza University of Iasi, Iasi, Romania

${ }^{27 \mathrm{~d}}$ National Institute for Research and Development of Isotopic and Molecular Technologies, Physics

Department, Cluj-Napoca, Romania

${ }^{27 \mathrm{e}}$ University Politehnica Bucharest, Bucharest, Romania

${ }^{27 \mathrm{f}}$ West University in Timisoara, Timisoara, Romania

${ }^{28 a}$ Faculty of Mathematics, Physics and Informatics, Comenius University, Bratislava, Slovak Republic

${ }^{28 \mathrm{~b}}$ Department of Subnuclear Physics, Institute of Experimental Physics of the Slovak Academy of Sciences, Kosice, Slovak Republic

${ }^{29}$ Physics Department, Brookhaven National Laboratory, Upton, New York, USA

${ }^{30}$ Departamento de Física, Universidad de Buenos Aires, Buenos Aires, Argentina

${ }^{31}$ California State University, California, USA

${ }^{32}$ Cavendish Laboratory, University of Cambridge, Cambridge, United Kingdom

${ }^{33 a}$ Department of Physics, University of Cape Town, Cape Town, South Africa

${ }^{33 \mathrm{~b}}$ Department of Mechanical Engineering Science, University of Johannesburg, Johannesburg, South Africa

${ }^{33 c}$ School of Physics, University of the Witwatersrand, Johannesburg, South Africa

${ }^{34}$ Department of Physics, Carleton University, Ottawa, Ontario, Canada

${ }^{35 a}$ Faculté des Sciences Ain Chock, Réseau Universitaire de Physique des Hautes Energies - Université Hassan II, Casablanca, Morocco

${ }^{35 \mathrm{~b}}$ Faculté des Sciences, Université Ibn-Tofail, Kénitra, Morocco

${ }^{35 \mathrm{c}}$ Faculté des Sciences Semlalia, Université Cadi Ayyad, LPHEA-Marrakech, Morocco

${ }^{35 \mathrm{~d}}$ Faculté des Sciences, Université Mohamed Premier and LPTPM, Oujda, Morocco

${ }^{35 \mathrm{e}}$ Faculté des sciences, Université Mohammed V, Rabat, Morocco

${ }^{36}$ CERN, Geneva, Switzerland

${ }^{37}$ Enrico Fermi Institute, University of Chicago, Chicago, Illinois, USA

${ }^{38}$ LPC, Université Clermont Auvergne, CNRS/IN2P3, Clermont-Ferrand, France

${ }^{39}$ Nevis Laboratory, Columbia University, Irvington, New York, USA

${ }^{40}$ Niels Bohr Institute, University of Copenhagen, Copenhagen, Denmark

${ }^{41 a}$ Dipartimento di Fisica, Università della Calabria, Rende, Italy

${ }^{41 \mathrm{~b}}$ INFN Gruppo Collegato di Cosenza, Laboratori Nazionali di Frascati, Frascati, Italy

${ }^{42}$ Physics Department, Southern Methodist University, Dallas, Texas, USA

${ }^{43}$ Physics Department, University of Texas at Dallas, Richardson, Texas, USA

${ }^{44 a}$ Department of Physics, Stockholm University, Sweden 
${ }^{44 \mathrm{~b}}$ Oskar Klein Centre, Stockholm, Sweden

${ }^{45}$ Deutsches Elektronen-Synchrotron DESY, Hamburg and Zeuthen, Germany

${ }^{46}$ Lehrstuhl für Experimentelle Physik IV, Technische Universität Dortmund, Dortmund, Germany

${ }^{47}$ Institut für Kern- und Teilchenphysik, Technische Universität Dresden, Dresden, Germany

${ }^{48}$ Department of Physics, Duke University, Durham, North Carolina, USA

${ }^{49}$ SUPA - School of Physics and Astronomy, University of Edinburgh, Edinburgh, United Kingdom

${ }^{50}$ INFN e Laboratori Nazionali di Frascati, Frascati, Italy

${ }^{51}$ Physikalisches Institut, Albert-Ludwigs-Universität Freiburg, Freiburg, Germany

${ }^{52}$ II. Physikalisches Institut, Georg-August-Universität Göttingen, Göttingen, Germany

${ }^{53}$ Département de Physique Nucléaire et Corpusculaire, Université de Genève, Genève, Switzerland

${ }^{54 a}$ Dipartimento di Fisica, Università di Genova, Genova, Italy

${ }^{54 \mathrm{~b}}$ INFN Sezione di Genova, Genova, Italy

${ }^{55}$ II. Physikalisches Institut, Justus-Liebig-Universität Giessen, Giessen, Germany

${ }^{56}$ SUPA - School of Physics and Astronomy, University of Glasgow, Glasgow, United Kingdom

${ }^{57}$ LPSC, Université Grenoble Alpes, CNRS/IN2P3, Grenoble INP, Grenoble, France

${ }^{58}$ Laboratory for Particle Physics and Cosmology, Harvard University, Cambridge, Massachusetts, USA

${ }^{59 a}$ Department of Modern Physics and State Key Laboratory of Particle Detection and Electronics,

University of Science and Technology of China, Hefei, China

${ }^{59 \mathrm{~b}}$ Institute of Frontier and Interdisciplinary Science and Key Laboratory of Particle Physics and Particle Irradiation (MOE), Shandong University, Qingdao, China

${ }^{59 \mathrm{c}}$ School of Physics and Astronomy, Shanghai Jiao Tong University, KLPPAC-MoE, SKLPPC, Shanghai, China

${ }^{59 \mathrm{~d}}$ Tsung-Dao Lee Institute, Shanghai, China

${ }^{60 a}$ Kirchhoff-Institut für Physik, Ruprecht-Karls-Universität Heidelberg, Heidelberg, Germany

${ }^{60 \mathrm{~b}}$ Physikalisches Institut, Ruprecht-Karls-Universität Heidelberg, Heidelberg, Germany

${ }^{61}$ Faculty of Applied Information Science, Hiroshima Institute of Technology, Hiroshima, Japan

${ }^{62 \mathrm{a}}$ Department of Physics, Chinese University of Hong Kong, Shatin, N.T., Hong Kong, China

${ }^{62 \mathrm{~b}}$ Department of Physics, University of Hong Kong, Hong Kong, China

${ }^{62 \mathrm{c}}$ Department of Physics and Institute for Advanced Study, Hong Kong University of Science and Technology, Clear Water Bay, Kowloon, Hong Kong, China

${ }^{63}$ Department of Physics, National Tsing Hua University, Hsinchu, Taiwan

${ }^{64}$ Department of Physics, Indiana University, Bloomington, Indiana, USA

${ }^{65 a}$ INFN Gruppo Collegato di Udine, Sezione di Trieste, Udine, Italy

${ }^{65 \mathrm{~b}}$ ICTP, Trieste, Italy

${ }^{65 \mathrm{c}}$ Dipartimento Politecnico di Ingegneria e Architettura, Università di Udine, Udine, Italy

${ }^{66 a}$ INFN Sezione di Lecce, Lecce, Italy

${ }^{66 \mathrm{~b}}$ Dipartimento di Matematica e Fisica, Università del Salento, Lecce, Italy

${ }^{67 a}$ INFN Sezione di Milano, Milano, Italy

${ }^{67 b}$ Dipartimento di Fisica, Università di Milano, Milano, Italy

${ }^{68 a}$ INFN Sezione di Napoli, Napoli, Italy

${ }^{68 \mathrm{~b}}$ Dipartimento di Fisica, Università di Napoli, Napoli, Italy

${ }^{69 a}$ INFN Sezione di Pavia, Pavia, Italy

${ }^{69 \mathrm{~b}}$ Dipartimento di Fisica, Università di Pavia, Pavia, Italy

${ }^{70 a}$ INFN Sezione di Pisa, Pisa, Italy

${ }^{70 \mathrm{~b}}$ Dipartimento di Fisica E. Fermi, Università di Pisa, Pisa, Italy

${ }^{71 a}$ INFN Sezione di Roma, Roma, Italy

${ }^{71 b}$ Dipartimento di Fisica, Sapienza Università di Roma, Roma, Italy

${ }^{72 a}$ INFN Sezione di Roma Tor Vergata, Roma, Italy

${ }^{72 b}$ Dipartimento di Fisica, Università di Roma Tor Vergata, Roma, Italy

${ }^{73 a}$ INFN Sezione di Roma Tre, Roma, Italy

${ }^{73 b}$ Dipartimento di Matematica e Fisica, Università Roma Tre, Roma, Italy

${ }^{74 a}$ INFN-TIFPA, Trento, Italy

${ }^{74 b}$ Università degli Studi di Trento, Trento, Italy

${ }^{75}$ Institut für Astro- und Teilchenphysik, Leopold-Franzens-Universität, Innsbruck, Austria

${ }^{76}$ University of Iowa, Iowa City, Iowa, USA

${ }^{77}$ Department of Physics and Astronomy, Iowa State University, Ames, Iowa, USA

${ }^{78}$ Joint Institute for Nuclear Research, Dubna, Russia

${ }^{79 a}$ Departamento de Engenharia Elétrica, Universidade Federal de Juiz de Fora (UFJF), Juiz de Fora, Brazil

${ }^{79 b}$ Universidade Federal do Rio De Janeiro COPPE/EE/IF, Rio de Janeiro, Brazil 
${ }^{79 c}$ Universidade Federal de São João del Rei (UFSJ), São João del Rei, Brazil

${ }^{79 \mathrm{~d}}$ Instituto de Física, Universidade de São Paulo, São Paulo, Brazil

${ }^{80}$ KEK, High Energy Accelerator Research Organization, Tsukuba, Japan

${ }^{81}$ Graduate School of Science, Kobe University, Kobe, Japan

${ }^{82 \mathrm{a}}$ AGH University of Science and Technology, Faculty of Physics and Applied Computer Science, Krakow, Poland

${ }^{82 \mathrm{~b}}$ Marian Smoluchowski Institute of Physics, Jagiellonian University, Krakow, Poland

${ }^{83}$ Institute of Nuclear Physics Polish Academy of Sciences, Krakow, Poland

${ }^{84}$ Faculty of Science, Kyoto University, Kyoto, Japan

${ }^{85}$ Kyoto University of Education, Kyoto, Japan

${ }^{86}$ Research Center for Advanced Particle Physics and Department of Physics, Kyushu University, Fukuoka, Japan

${ }^{87}$ Instituto de Física La Plata, Universidad Nacional de La Plata and CONICET, La Plata, Argentina

${ }^{88}$ Physics Department, Lancaster University, Lancaster, United Kingdom

${ }^{89}$ Oliver Lodge Laboratory, University of Liverpool, Liverpool, United Kingdom

${ }^{90}$ Department of Experimental Particle Physics, Jožef Stefan Institute and Department of Physics, University of Ljubljana, Ljubljana, Slovenia

${ }^{91}$ School of Physics and Astronomy, Queen Mary University of London, London, United Kingdom

${ }^{92}$ Department of Physics, Royal Holloway University of London, Egham, United Kingdom

${ }^{93}$ Department of Physics and Astronomy, University College London, London, United Kingdom

${ }^{94}$ Louisiana Tech University, Ruston, Louisiana, USA

${ }^{95}$ Fysiska institutionen, Lunds universitet, Lund, Sweden

${ }^{96}$ Centre de Calcul de l'Institut National de Physique Nucléaire et de Physique des Particules (IN2P3), Villeurbanne, France

${ }^{97}$ Departamento de Física Teorica C-15 and CIAFF, Universidad Autónoma de Madrid, Madrid, Spain

${ }^{98}$ Institut für Physik, Universität Mainz, Mainz, Germany

${ }^{99}$ School of Physics and Astronomy, University of Manchester, Manchester, United Kingdom

${ }^{100}$ CPPM, Aix-Marseille Université, CNRS/IN2P3, Marseille, France

${ }^{101}$ Department of Physics, University of Massachusetts, Amherst, Massachusetts, USA

${ }^{102}$ Department of Physics, McGill University, Montreal, Quebec, Canada

${ }^{103}$ School of Physics, University of Melbourne, Victoria, Australia

${ }^{104}$ Department of Physics, University of Michigan, Ann Arbor, Michigan, USA

${ }^{105}$ Department of Physics and Astronomy, Michigan State University, East Lansing, Michigan, USA

${ }^{106}$ B.I. Stepanov Institute of Physics, National Academy of Sciences of Belarus, Minsk, Belarus

${ }^{107}$ Research Institute for Nuclear Problems of Byelorussian State University, Minsk, Belarus

${ }^{108}$ Group of Particle Physics, University of Montreal, Montreal, Quebec, Canada

${ }^{109}$ P.N. Lebedev Physical Institute of the Russian Academy of Sciences, Moscow, Russia

${ }^{110}$ Institute for Theoretical and Experimental Physics of the National Research Centre Kurchatov Institute,

Moscow, Russia

${ }^{111}$ National Research Nuclear University MEPhI, Moscow, Russia

${ }^{112}$ D.V. Skobeltsyn Institute of Nuclear Physics, M.V. Lomonosov Moscow State University, Moscow, Russia

${ }^{113}$ Fakultät für Physik, Ludwig-Maximilians-Universität München, München, Germany

${ }^{114}$ Max-Planck-Institut für Physik (Werner-Heisenberg-Institut), München, Germany

${ }^{115}$ Nagasaki Institute of Applied Science, Nagasaki, Japan

${ }^{116}$ Graduate School of Science and Kobayashi-Maskawa Institute, Nagoya University, Nagoya, Japan

${ }^{117}$ Department of Physics and Astronomy, University of New Mexico, Albuquerque, New Mexico, USA

${ }^{118}$ Institute for Mathematics, Astrophysics and Particle Physics, Radboud University Nijmegen/Nikhef, Nijmegen, Netherlands

${ }^{119}$ Nikhef National Institute for Subatomic Physics and University of Amsterdam, Amsterdam, Netherlands

${ }^{120}$ Department of Physics, Northern Illinois University, DeKalb, Illinois, USA

${ }^{121 \mathrm{a}}$ Budker Institute of Nuclear Physics and NSU, SB RAS, Novosibirsk, Russia

${ }^{121 \mathrm{~b}}$ Novosibirsk State University Novosibirsk, Russia

${ }^{122}$ Institute for High Energy Physics of the National Research Centre Kurchatov Institute, Protvino, Russia

${ }^{123}$ Department of Physics, New York University, New York, New York, USA

${ }^{124}$ Ochanomizu University, Otsuka, Bunkyo-ku, Tokyo, Japan

${ }^{125}$ The Ohio State University, Columbus, Ohio, USA

${ }^{126}$ Faculty of Science, Okayama University, Okayama, Japan

${ }^{127}$ Homer L. Dodge Department of Physics and Astronomy, University of Oklahoma,

Norman, Oklahoma, USA 
${ }^{128}$ Department of Physics, Oklahoma State University, Stillwater, Oklahoma, USA

${ }^{129}$ Palacký University, RCPTM, Joint Laboratory of Optics, Olomouc, Czech Republic

${ }^{130}$ Center for High Energy Physics, University of Oregon, Eugene, Oregon, USA

${ }^{131}$ LAL, Université Paris-Sud, CNRS/IN2P3, Université Paris-Saclay, Orsay, France

${ }^{132}$ Graduate School of Science, Osaka University, Osaka, Japan

${ }^{133}$ Department of Physics, University of Oslo, Oslo, Norway

${ }^{134}$ Department of Physics, Oxford University, Oxford, United Kingdom

${ }^{135}$ LPNHE, Sorbonne Université, Paris Diderot Sorbonne Paris Cité, CNRS/IN2P3, Paris, France

${ }^{136}$ Department of Physics, University of Pennsylvania, Philadelphia, Pennsylvania, USA

${ }^{137}$ Konstantinov Nuclear Physics Institute of National Research Centre "Kurchatov Institute", PNPI, St. Petersburg, Russia

${ }^{138}$ Department of Physics and Astronomy, University of Pittsburgh, Pittsburgh, Pennsylvania, USA

${ }^{139 a}$ Laboratório de Instrumentação e Física Experimental de Partículas - LIP, Portugal

${ }^{139 b}$ Departamento de Física, Faculdade de Ciências, Universidade de Lisboa, Lisboa, Portugal

${ }^{139 \mathrm{c}}$ Departamento de Física, Universidade de Coimbra, Coimbra, Portugal

${ }^{139 d}$ Centro de Física Nuclear da Universidade de Lisboa, Lisboa, Portugal

${ }^{139 \mathrm{e}}$ Departamento de Física, Universidade do Minho, Braga, Portugal

${ }^{139 f}$ Universidad de Granada, Granada (Spain), Spain

${ }^{139 \mathrm{~g}}$ Dep Física and CEFITEC of Faculdade de Ciências e Tecnologia, Universidade Nova de Lisboa, Caparica, Portugal

${ }^{140}$ Institute of Physics of the Czech Academy of Sciences, Prague, Czech Republic

${ }^{141}$ Czech Technical University in Prague, Prague, Czech Republic

${ }^{142}$ Charles University, Faculty of Mathematics and Physics, Prague, Czech Republic

${ }^{143}$ Particle Physics Department, Rutherford Appleton Laboratory, Didcot, United Kingdom

${ }^{144}$ IRFU, CEA, Université Paris-Saclay, Gif-sur-Yvette, France

${ }^{145}$ Santa Cruz, Institute for Particle Physics, University of California Santa Cruz, Santa Cruz, California, USA

${ }^{146 a}$ Departamento de Física, Pontificia Universidad Católica de Chile, Santiago, Chile

${ }^{146 \mathrm{~b}}$ Departamento de Física, Universidad Técnica Federico Santa María, Valparaíso, Chile

${ }^{147}$ Department of Physics, University of Washington, Seattle, Washington, USA

${ }^{148}$ Department of Physics and Astronomy, University of Sheffield, Sheffield, United Kingdom

${ }^{149}$ Department of Physics, Shinshu University, Nagano, Japan

${ }^{150}$ Department Physik, Universität Siegen, Siegen, Germany

${ }^{151}$ Department of Physics, Simon Fraser University, Burnaby, British Columbia, Canada

${ }^{152}$ SLAC National Accelerator Laboratory, Stanford, California, USA

${ }^{153}$ Physics Department, Royal Institute of Technology, Stockholm, Sweden

${ }^{154}$ Departments of Physics and Astronomy, Stony Brook University, Stony Brook, New York, USA

${ }^{155}$ Department of Physics and Astronomy, University of Sussex, Brighton, United Kingdom

${ }^{156}$ School of Physics, University of Sydney, Sydney, Australia

${ }^{157}$ Institute of Physics, Academia Sinica, Taipei, Taiwan

${ }^{158 a}$ E. Andronikashvili Institute of Physics, Iv. Javakhishvili Tbilisi State University, Tbilisi, Georgia

${ }^{158 \mathrm{~b}}$ High Energy Physics Institute, Tbilisi State University, Tbilisi, Georgia

${ }^{159}$ Department of Physics, Technion, Israel Institute of Technology, Haifa, Israel

${ }^{160}$ Raymond and Beverly Sackler School of Physics and Astronomy, Tel Aviv University, Tel Aviv, Israel

${ }^{161}$ Department of Physics, Aristotle University of Thessaloniki, Thessaloniki, Greece

${ }^{162}$ International Center for Elementary Particle Physics and Department of Physics, University of Tokyo, Tokyo, Japan

${ }^{163}$ Graduate School of Science and Technology, Tokyo Metropolitan University, Tokyo, Japan

${ }^{164}$ Department of Physics, Tokyo Institute of Technology, Tokyo, Japan

${ }^{165}$ Tomsk State University, Tomsk, Russia

${ }^{166}$ Department of Physics, University of Toronto, Toronto, Ontario, Canada

${ }^{167 a}$ TRIUMF, Vancouver, British Columbia, Canada

${ }^{167 b}$ Department of Physics and Astronomy, York University, Toronto, Ontario, Canada

${ }^{168}$ Division of Physics and Tomonaga Center for the History of the Universe, Faculty of Pure and Applied Sciences, University of Tsukuba, Tsukuba, Japan

${ }^{169}$ Department of Physics and Astronomy, Tufts University, Medford, Massachusetts, USA

${ }^{170}$ Department of Physics and Astronomy, University of California Irvine, Irvine, California, USA

${ }^{171}$ Department of Physics and Astronomy, University of Uppsala, Uppsala, Sweden

${ }^{172}$ Department of Physics, University of Illinois, Urbana, Illinois, USA

${ }^{173}$ Instituto de Física Corpuscular (IFIC), Centro Mixto Universidad de Valencia - CSIC, Valencia, Spain 


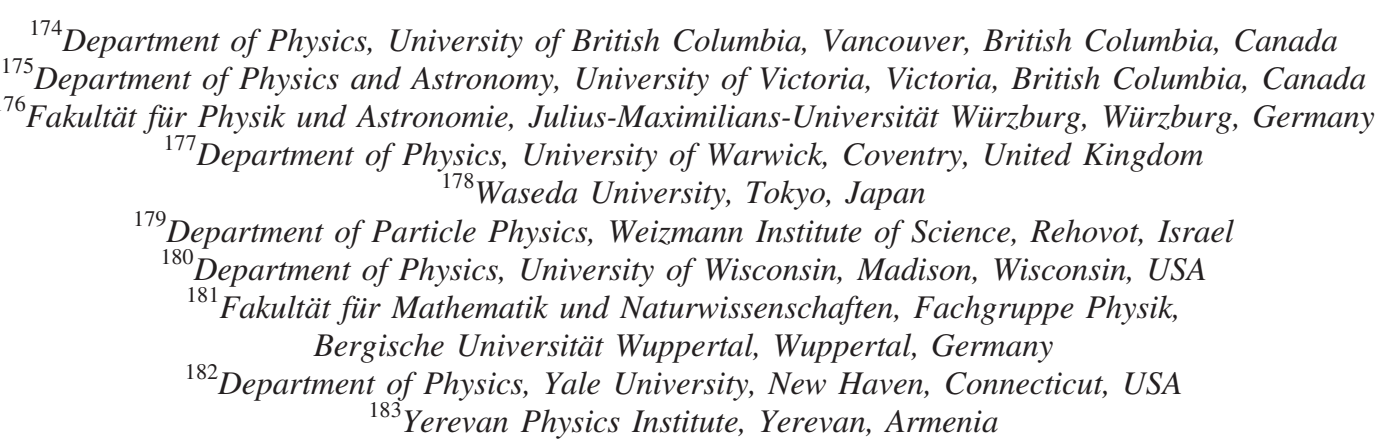

${ }^{\mathrm{a}}$ Deceased.

${ }^{\mathrm{b}}$ Also at Department of Physics, King's College London, London, United Kingdom.

${ }^{c}$ Also at Institute of Physics, Azerbaijan Academy of Sciences, Baku, Azerbaijan.

${ }^{\mathrm{d}}$ Also at TRIUMF, Vancouver, British Columbia, Canada.

${ }^{\mathrm{e}}$ Also at Department of Physics and Astronomy, University of Louisville, Louisville, Kentucky, USA.

${ }^{\mathrm{f}}$ Also at Physics Department, An-Najah National University, Nablus, Palestine.

${ }^{\mathrm{g}}$ Also at Department of Physics, California State University, Fresno, USA.

${ }^{\mathrm{h}}$ Also at Department of Physics, University of Fribourg, Fribourg, Switzerland.

${ }^{\mathrm{i}}$ Also at Departament de Fisica de la Universitat Autonoma de Barcelona, Barcelona, Spain.

${ }^{\mathrm{j}}$ Also at Tomsk State University, Tomsk, and Moscow Institute of Physics and Technology State University, Dolgoprudny, Russia.

${ }^{\mathrm{k}}$ Also at The Collaborative Innovation Center of Quantum Matter (CICQM), Beijing, China.

${ }^{1}$ Also at Universita di Napoli Parthenope, Napoli, Italy.

${ }^{\mathrm{m}}$ Also at Institute of Particle Physics (IPP), Canada.

${ }^{\mathrm{n}}$ Also at II. Physikalisches Institut, Georg-August-Universität Göttingen, Göttingen, Germany.

${ }^{\circ}$ Also at Horia Hulubei National Institute of Physics and Nuclear Engineering, Bucharest, Romania.

${ }^{\mathrm{p}}$ Also at CPPM, Aix-Marseille Université, CNRS/IN2P3, Marseille, France.

${ }^{\mathrm{q}}$ Also at Department of Physics, St. Petersburg State Polytechnical University, St. Petersburg, Russia.

${ }^{\mathrm{r}}$ Also at Borough of Manhattan Community College, City University of New York, New York, New York, USA.

${ }^{\mathrm{s}}$ Also at Department of Financial and Management Engineering, University of the Aegean, Chios, Greece.

${ }^{\mathrm{t}}$ Also at Centre for High Performance Computing, CSIR Campus, Rosebank, Cape Town, South Africa.

"Also at Department of Physics, California State University, East Bay, Hayward, California, USA.

${ }^{v}$ Also at Institucio Catalana de Recerca i Estudis Avancats, ICREA, Barcelona, Spain.

${ }^{w}$ Also at Department of Physics, University of Michigan, Ann Arbor, Michigan, USA.

${ }^{\mathrm{x}}$ Also at LAL, Université Paris-Sud, CNRS/IN2P3, Université Paris-Saclay, Orsay, France.

${ }^{y}$ Also at Graduate School of Science, Osaka University, Osaka, Japan.

${ }^{\mathrm{z}}$ Also at Physikalisches Institut, Albert-Ludwigs-Universität Freiburg, Freiburg, Germany.

${ }^{\text {aa }}$ Also at Institute for Mathematics, Astrophysics and Particle Physics, Radboud University Nijmegen/Nikhef, Nijmegen, Netherlands.

${ }^{\mathrm{bb}}$ Also at Institute of Theoretical Physics, Ilia State University, Tbilisi, Georgia.

${ }^{\mathrm{cc}}$ Also at CERN, Geneva, Switzerland.

${ }^{\mathrm{dd}}$ Also at Manhattan College, New York, New York, USA.

${ }^{\mathrm{ee}}$ Also at Hellenic Open University, Patras, Greece.

${ }^{\mathrm{ff}}$ Also at LPNHE, Sorbonne Université, Paris Diderot Sorbonne Paris Cité, CNRS/IN2P3, Paris, France.

${ }^{g g}$ Also at The City College of New York, New York, New York, USA.

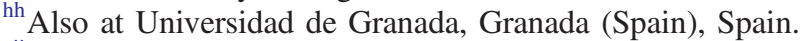

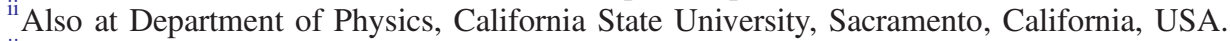

${ }^{\mathrm{jj}}$ Also at Moscow Institute of Physics and Technology State University, Dolgoprudny, Russia.

${ }^{k k}$ Also at Département de Physique Nucléaire et Corpusculaire, Université de Genève, Genève, Switzerland.

${ }^{11}$ Also at Department of Physics and Astronomy, University of Sheffield, Sheffield, United Kingdom.

${ }^{\mathrm{mm}}$ Also at Louisiana Tech University, Ruston, Louisiana, USA.

${ }^{\mathrm{nn}}$ Also at School of Physics, Sun Yat-sen University, Guangzhou, China.

${ }^{\circ o}$ Also at Institute for Nuclear Research and Nuclear Energy (INRNE) of the Bulgarian Academy of Sciences, Sofia, Bulgaria.

${ }^{\mathrm{pp}}$ Also at Faculty of Physics, M.V. Lomonosov Moscow State University, Moscow, Russia.

${ }^{\mathrm{qq}}$ Also at Department of Applied Physics and Astronomy, University of Sharjah, Sharjah, United Arab Emirates.

${ }^{\mathrm{rr}}$ Also at Institut für Experimentalphysik, Universität Hamburg, Hamburg, Germany.

${ }^{\text {ss }}$ Also at National Research Nuclear University MEPhI, Moscow, Russia. 
${ }^{\text {tt }}$ Also at Institute for Particle and Nuclear Physics, Wigner Research Centre for Physics, Budapest, Hungary.

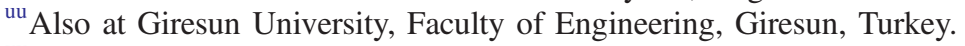

${ }^{\mathrm{vv}}$ Also at Institute of Physics, Academia Sinica, Taipei, Taiwan. 